\title{
University of
}

Roehampton

London

\section{PSYCHD}

\section{Helpful and unhelpful processes in psychological therapy for female substance users an interpretative phenomenological analysis}

Halsall, Jane Louise

Award date:

2018

Awarding institution:

University of Roehampton

\section{General rights}

Copyright and moral rights for the publications made accessible in the public portal are retained by the authors and/or other copyright owners and it is a condition of accessing publications that users recognise and abide by the legal requirements associated with these rights.

- Users may download and print one copy of any publication from the public portal for the purpose of private study or research.

- You may not further distribute the material or use it for any profit-making activity or commercial gain

- You may freely distribute the URL identifying the publication in the public portal ? 
Helpful and unhelpful processes in psychological therapy for female substance users: An Interpretative Phenomenological Analysis.

\section{JANE LOUISE HALSALL}

A thesis submitted in partial fulfilment for the degree of Professional Doctorate in Counselling Psychology

Department of Psychology

University of Roehampton

2017 
Helpful and unhelpful processes in psychological therapy for female substance users

\section{Declaration}

I hereby declare that the work submitted in this dissertation is fully the result of my own investigation, except where otherwise stated.

Name: Jane Halsall

Date: December 2017 
Helpful and unhelpful processes in psychological therapy for female substance users

\section{ACKNOWLEDGEMENTS}

This thesis is dedicated to my parents, Michael and Carol Halsall, and to my beautiful daughter, Nyla Halsall and son, Luca. Thank you also to my partner Mikele Loffredo, without your invaluable support and belief in me, this thesis would not have been completed. Thank you to my academic supervisors, Professor Mick Cooper and Dr Joel Vos for their guidance and support in this challenging journey. Finally, thank you to everyone who directly or indirectly contributed to my inspiration to finish this thesis and believed in me. I would also like to dedicate this thesis to the eight women who kindly and openly shared their stories with me and anyone else affected by substance use disorder. 
Helpful and unhelpful processes in psychological therapy for female substance users

\begin{abstract}
Addiction is a complex phenomenon characterised by a loss of control and compulsive, habitual behaviour. Since there is no single, specific cause for addiction, there is no single standard treatment. The prevalence of substance use disorder is on the increase and treatment success rates are low. Addiction literature is dominated by quantitative methodologies investigating biomedical and evidence based psychological practices. As such, a deficit of qualitative research exists and limited attention has been placed on understanding women's perceptions of psychological treatment for substance use disorder (SUD). This phenomenological study explores the experience of psychological therapy from the perspective of women diagnosed with SUD under the DSM-V criteria. It aims to identify aspects of the therapeutic process that they found helpful and unhelpful.
\end{abstract}

Eight women participated in semi-structured interviews, which were analysed using interpretative phenomenological analysis (IPA). Three superordinate themes were identified: 'What words can't express; finding another language,' 'Identification with my therapist,' and 'Getting towards acceptance'. The findings indicated that the interpersonal relationship with their therapist and the women's emotional affect impacted their level of engagement. In the narratives, resistance and defensive behaviour were identified as a barrier when finding their voice at the start of therapy. This shifted as the therapeutic alliance strengthened. An unexpected finding was the importance of women's perceptions of having a therapist who was also in 'recovery' from substance use disorder. Clinical and research implications include: reassessing therapeutic interventions for this client group and an exploration on how shame can be worked with relationally. In light of this, suggestions are also made for how clinicians might maximise early engagement with clients and potentially adapt treatment plans to 
Helpful and unhelpful processes in psychological therapy for female substance users

fit a client's individual needs. 
Helpful and unhelpful processes in psychological therapy for female substance users

\section{TABLE OF CONTENTS:}

PAGE NUMBER

Acknowledgements.......................................................

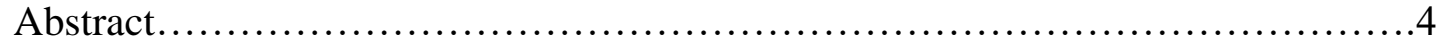

\section{CHAPTER ONE: Introduction}

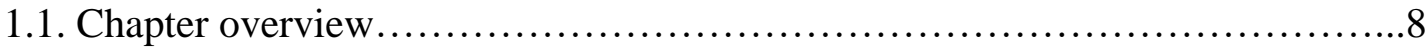

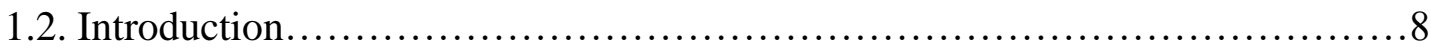

1.3. Addiction and Counselling Psychology..................................... 12

1.4. Defining addiction..................................................... 13

1.5. Treatment modalities....................................................... 17

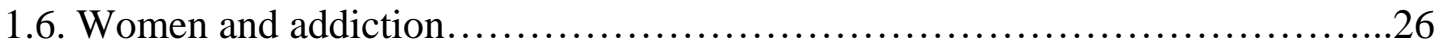

\section{CHAPTER TWO: Literature Review}

2.0. Existing research in the field ............................................ 30

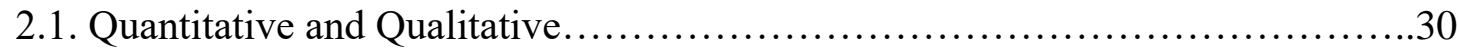

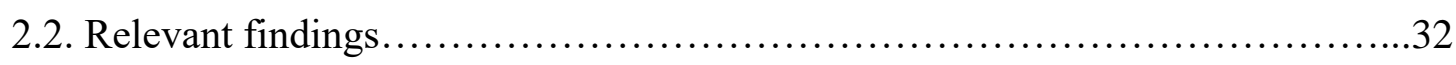

2.3. Overview of aims and the potential contribution of the study..................39

\section{CHAPTER THREE: Methodology and Method}

3.1 Research Question.....................................................42

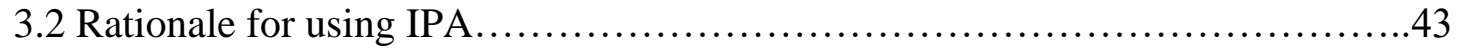

3.3 Alternative qualitative methodologies.....................................45

3.4 Epistemological standpoint.........................................46

3.5 IPA methodology and philosophical underpinnings........................48

3.5.1 Phenomenology ...................................................... 49

3.5.2 Hermeneutics................................................... 52

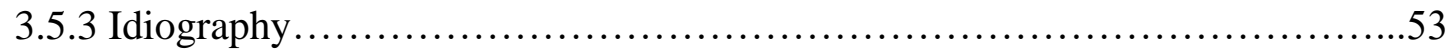

3.6 Research Process.......................................................53

3.6.1.Participants........................................................ 54

3.6.2 Recruitment ....................................................55

3.6.3 Inclusion and exclusion criteria......................................57

3.6.4 Data collection......................................................59

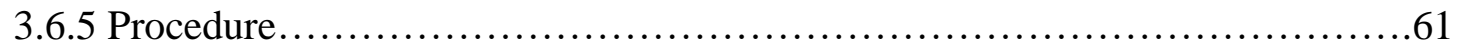

3.6.6 Ethical considerations.............................................62 
Helpful and unhelpful processes in psychological therapy for female substance users

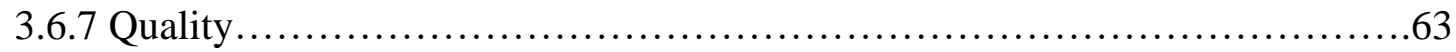

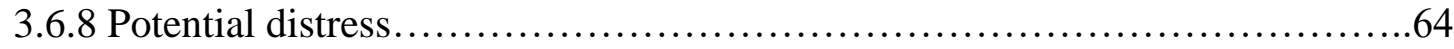

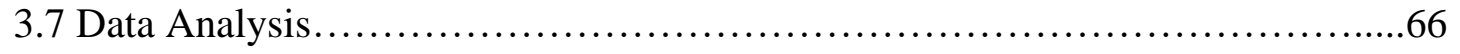

3.7.1 Individual case analysis...........................................66

3.7.2 Emergent themes...............................................68

3.7.3 Cross case analysis .................................................69

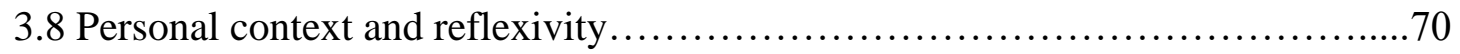

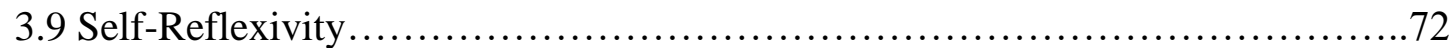

\section{CHAPTER FOUR: Findings}

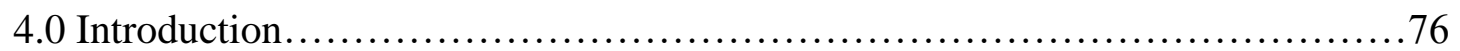

4.1 Superordinate Theme 1: "What words can't express: finding another

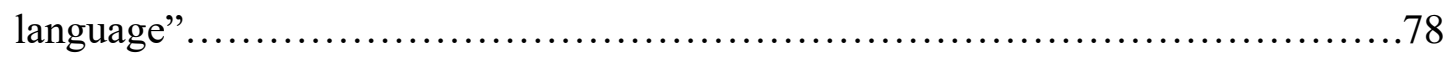

4.2 Superordinate Theme 2: 'Identification with my

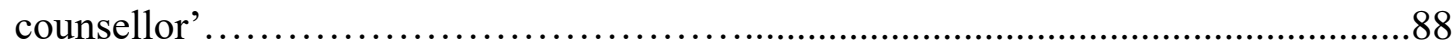

4.3 Superordinate Theme 3: 'Getting towards

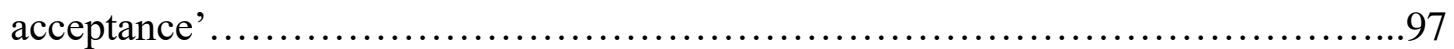

\section{CHAPTER FIVE: Discussion}

5.0 Chapter overview...................................................... 107

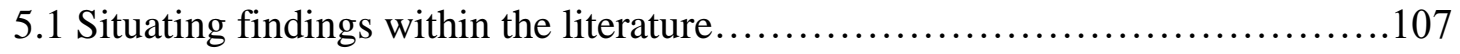

5.2 Evaluation of this study............................................... 117

5.3 A critique of Interpretive Phenomenological Analysis.......................117

5.4 Clinical and research implications for Counselling Psychologists..............121

5.5 Suggestions for future research........................................ 123

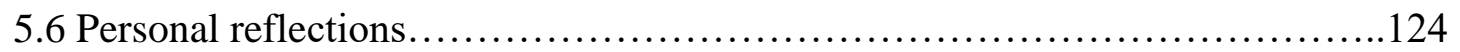

5.7 Conclusion......................................................... 128

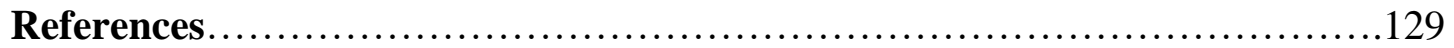

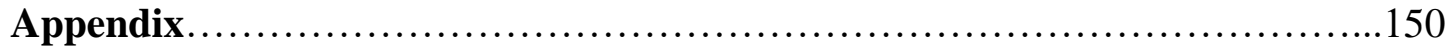


Helpful and unhelpful processes in psychological therapy for female substance users

\section{CHAPTER ONE: \\ Introduction}

\subsection{Chapter Overview}

This chapter provides insight into the prevalence of drug and alcohol addiction and the complexity of defining this affliction. It will begin by exploring the multitude of definitions and theories around addiction and how it affects the current provision of services in the United Kingdom (UK). Following this, a focus upon women and addiction will inform what research is currently available and justify why this research is needed. The chapter will conclude with how addiction is explored within the framework of counselling psychology.

\subsection{Introduction}

Addiction is a major concern globally with an estimated 29 million people drug dependent (United Nations World Drug Report, 2017). The National Treatment Agency (NTA, 2016) reports that in 2015/16, 288,843 individuals made contact with addiction services for substance use disorder, 138,081 commenced treatment (psychological therapy) and 127,080 completed treatment. Of those that completed treatment, 64,166 were classified as free of dependence (NTA, 2016). These low positive outcome figures suggest that the current system for treating substance use disorder is failing and that the continued development of improved treatments and research is required.

Exploration of the type of client presenting for substance use disorder treatment provided an insight into the profile of those individuals accessing treatment. The 2015/16 Adult Substance Misuse Statistics Report, taken from the National Drug 
Helpful and unhelpful processes in psychological therapy for female substance users

Treatment Monitoring system (NDTMS, 2016) within England, found that men made up $70 \%$ of the total number presenting for treatment with $85 \%$ classified as White British. Clients dependent on opiates made up the largest proportion of the total numbers in treatment ( $52 \%$ of the total), followed by alcohol (29\%). Of the $52 \%$ opiate users, $49 \%$ also had a dependence on crack cocaine. Despite opiate users presenting as the largest group, they represented the lowest rate of successful treatment completions (28\%). This was compared to the other three substance groups (ranging from 56\% for non-opiate and alcohol clients to $62 \%$ for alcohol only clients).

These data reveal that the predominant profile of those seeking treatment in the UK, are white British male opiate users. Although studies have reported a gender difference with men using drugs more than women, this difference is getting significantly smaller, making women's addiction problems an increasingly important phenomenon to consider (Bawor et al., 2015). Women currently represent $30 \%$ of the total number of clients presenting for treatment, however, research from the NTA show that this figure is on the increase and expected to become equal to men in the near future (NTA, 2016). This thesis focuses on women due to the expectation that the numbers of women presenting for treatment is likely to rise and that their subjective experience of psychological therapy for substance use disorder is yet to be explored. With this in mind, this thesis will hope to prepare for this increase and provide a voice to this otherwise marginalised group.

The impact of addiction reaches far beyond those in psychological treatment; destroying families, affecting welfare, with ensuing pressure on the UK criminal justice system and the NHS (NICE, 2016). Since the Centre of Social Justice commissioned a report titled No quick fix in 2013 exposing the depth of Britain's drug and alcohol 
Helpful and unhelpful processes in psychological therapy for female substance users

problems, there is a continued interest in the management and treatment of this client group. The current financial cost to taxpayers in the UK is $£ 15.4$ billion pounds annually (ABC Money, 2017) and $£ 488$ million for the NHS to treat. Yet we know that individuals are returning to treatment and only a small proportion reach a place of longterm recovery.

At a time when drug policies are failing, financial cuts are being implemented and costs to services are increasing, there is a need to focus on the psychological support currently available and whether it is effective. However, the role played by treatment in helping addicts to become abstinent and the evidence of effectiveness of specific treatments is often difficult to interpret (Gossop, 2004). The variety of addiction treatments, interventions and procedures can create difficulties when drawing comparisons and measuring effectiveness. However, there is increasing agreement that research should pay greater attention to questions of process, specifically placing a focus upon how treatment works and how it can be improved (Prendergast et al, 2002; Simpson, 2004, Jhangee, 2014). Hence, this study aims to develop an in-depth understanding of what women with substance use disorder feel is helpful and unhelpful during their psychological treatment, which a phenomenological approach can provide.

Despite the prevalence of this condition and the enormity of the consequences, such as, family breakdown, economic difficulties and health issues that result from substance use disorder, the lack of effective solutions, screening and early intervention is sustaining this issue (Centre of Addiction and Substance Abuse, 2012). In addition, the vast majority of people in need of psychological treatment do not receive evidencebased care (Altman, 2012). Research has also shown that the majority of practitioners working with addiction are not equipped with the knowledge, skills or credentials 
Helpful and unhelpful processes in psychological therapy for female substance users

necessary to provide the full range of evidence-based services to treat this disease (CASA, 2012). The high treatment failure rates have been attributed to internal qualities of the service user, including; fears about the impact on their employment, a negative relationship with their therapist, denial about their dependence, and an absence of dual diagnosis treatment (McGraw, 2016).

Some studies have reported that rates of return to substance use continue to be high post treatment, with clients often relapsing a short time after treatment ends (Gossop et al., 2002, Sack, 2012). Approximately half of all individuals with drug and alcohol dependency who attempt sobriety return to heavy use post treatment (Sack, 2012). This highlights the often seen cyclical process of addiction and how the majority of individuals remain within the system. An exploration of why people relapse, found shame and guilt and external (including interpersonal) events as contributing factors (American Addiction Centres, 2017). The aforementioned studies on the cyclical process and reasons for relapse may be affiliated with the process of psychological therapy, hence the argument to explore that aspect in further detail, to see if access, retention and efficacy rates can be improved.

In this thesis, I examine aspects of treatment for substance use disorder in women who have not received a paucity of attention in the literature. I aim to explore how women make sense of their psychological treatment for SUD, with a focus on the process of treatment, rather than the outcomes of treatment, identifying their reported helpful and unhelpful factors in psychological therapy.

Even though a vast body of literature on addiction exists, research from the perspectives of substance users "remains scant" (Nordjfaern et al, 2010 p.3, Watson \& Parke, 2009, Merrick et al, 2012,). To reverse this dynamic, there is a need for research 
Helpful and unhelpful processes in psychological therapy for female substance users

that has been informed by the service users' own accounts in the context of their therapeutic recovery. This insight will provide greater depth and understanding about the needs of this client group from their perspective. Such findings will also contribute to raising awareness among counselling psychologists and other psychological practitioners to the obstacles faced by clients engaged in psychological therapy for SUD and how to overcome these complexities.

\subsection{Addiction and Counselling Psychology}

In this section, it is proposed that the problematic nature of addiction needs to be explored in the context of the counselling psychology profession. Counselling psychology is a relatively new profession and was first constituted as a separate division of the British Psychological Society (BPS) in 1982. It draws upon and seeks to develop phenomenological models of practice and enquiry in addition to that of scientific psychology, respecting first person accounts as valid in their own terms (The Division of Counselling Psychology, BPS, 2016). This BPS description suggests that counselling psychology is situated within two domains: practice and knowledge. As scientistpractitioners, counselling psychologists are often called to work within medical and scientific settings that promote positivist and empirical ideas whilst also valuing subjectivity, intersubjectivity, the therapeutic relationship, meaning making, pluralism and anti-discriminatory practice (BPS, 2015).

The medical, positivist approach places an emphasis on making a diagnostic evaluation of SUD as evidenced by the DSM-V (DSM-5, 2013). However, this process challenges the underlying principle of counselling psychology, as it takes a non-holistic look at the client, reducing human experience to a collection of symptoms. Instead, counselling psychologists are expected to challenge modernist assumptions about 
Helpful and unhelpful processes in psychological therapy for female substance users

human experience, both within medical and humanistic perspectives, and value pluralism (Rizq, 2007). Pluralism ascribes to the idea that knowledge is relevant and that there are many versions of reality and truths (Cooper and McLeod, 2011). Even though counselling psychologists working with SUD clients are often expected to work within a socially constructed diagnosis (West, 2006) embracing the psychiatric model is useful if an individual has a diagnosis of comorbidity and unable to ameliorate their symptoms without the assistance of medication. As mood disorders can increase vulnerability to SUD, a medical intervention can reduce subsequent drug use (NIDA, 2012). Therefore, it is important to find the balance between both perspectives. This research aims to investigate what women with SUD find helpful and unhelpful in psychological therapy by exploring the individual from a holistic and pluralistic perspective within that process. In giving women a voice in this way, the approach fits with the humanistic values of counselling psychology.

\subsection{Defining Addiction}

The aim of this section is to address the complexity of defining addiction and its implications for treatment models. It can be questioned whether the lack of standardised terminology compromises effective interventions. In analysing the interplay between the conceptualisation of addiction and the varied approaches to treatment, the complexity of labelling addiction as a 'disease' will be explored demonstrating the struggle to define SUD in a way that universally conceptualises all those affected.

According to the Oxford English Dictionary, the term 'addiction' is etymologically derived from Latin addictlls and addicere, meaning 'deliver, yield, devote', from ad'to' + dicere 'say, declare' (Oxford English Dictionary, 2017). Originally it was used in the domain of Roman law to designate a formal giving over or delivery by sentence of 
Helpful and unhelpful processes in psychological therapy for female substance users

court, hence, indicating a surrender, or dedication to a master. Progressing from the concept of being 'bound or devoted to someone,' addiction is currently defined as a primary, chronic disease of brain reward, motivation, memory and related circuitry. Dysfunction in these circuits leads to characteristic biological, psychological, social and spiritual manifestations. This is reflected in an individual pathologically pursuing reward and/or relief by substance use and other behaviours (American Society of Addictive Medicine, ASAM, 2011). In relation to the concept of addiction within this thesis, SUD is written about within a diagnostic framework as utilised by service treatment providers, and this thesis will discuss the challenges this holds when navigating a counselling psychologist identity.

In researching the vast literature, it is clear a number of discourses defining addiction exist and meanings can be subjective to the individual. Before discussing these, it is worth acknowledging how the term 'addiction' continues to hold stigmatising connotations for this client group (Larkin and Griffiths, 2006). Past descriptions that labelled addicts as 'morally corrupt and socially impoverished' (Shaffer, 1986 p.208, as cited by Larkin et al, 2005) set the tone for negative perceptions of addiction, and the view that addiction is a moral weakness. Larkin and Griffith (2006) talk about challenging this label and suggest reconceptualising the term 'addiction' to something less distressing for those affected.

Addiction is an abstract concept, socially defined, that varies according to the functions or purposes it fulfills, leaving it open to individual opinion that may not be correct (West, 2006, Hammersley \& Reid, 2002). This means that its boundaries are unclear. The transmutation process SUD has been subjected to over the years has also created linguistic and conceptual challenges, such as changing definitions of addiction 
Helpful and unhelpful processes in psychological therapy for female substance users

in The Diagnostic and Statistical Manual V (DSM-5; APA, 2013) from abuse, dependence to substance use disorder (SUD) (APA, 2016). Terms used to describe different levels of involvement with addictive substances, such as: experimentation, use, misuse, excessive use, abuse, dependence and addiction lack precision, obscuring important differences in the nature and severity of the illness and complicating our ability to intervene and treat it effectively. Even the word "treatment" lacks precision with regard to addiction, since historically it has been used to refer to a host of interventions, many of which are not based in the clinical and scientific evidence as are treatments for other diseases (Gossop, 2006).

Addiction has been described as a behavioural manifestation of a chronic condition in which a reward seeking behaviour has become 'out of control' (West, 2006). However, some individuals may present with a clear addiction and others with addiction-like symptoms and each of these challenges to an agreed definition have implications on treatment. The diversity of theoretical perspectives available within the current psychological literature and research in relation to SUD means the different constructions result in different therapeutic applications.

In reference to the disease model, the DSM-V (DSM-5; APA, 2013) constitutes the primary diagnostic tool for Mental Health disorders utilised by counselling psychologists and other health clinicians, to understand psychological difficulties (NIDA, 2016). The fifth edition has merged the previous categories of substance abuse and substance dependence to define 'addiction' as a single disorder - (SUD) and is measured on a continuum from mild to severe. A mild SUD is the presence of two to three symptoms, a moderate, four to five symptoms, and severe, six or more symptoms (DSM-5; APA, 2013). 
Helpful and unhelpful processes in psychological therapy for female substance users

In the DSM-5 (APA, 2013) the diagnosis of SUD is based on a pathological pattern of behaviours related to use of the substance. The symptoms include: 1) substance is taken in larger amounts or over a longer period than originally intended; 2) the individual expresses a desire to cut down or regulate use but is unsuccessful in their efforts to decrease; 3) a great deal of time is spent in activities necessary to obtain the substance; 4) craving is manifested by an intense desire or urge for the drug at any time; 5) a failure to fulfill major role obligations at work / school; 6) recurrent social or interpersonal problems caused by the effects of the substance; 7) important activities reduced or given up because of substance use; 8) recurrent use in physically hazardous situations; 9) continue use despite knowledge of a persistent physical or psychological problem caused or exacerbated by substance; 10) tolerance - requiring an increased dose to get the same effect; 11) withdrawal symptoms (DSM-5; APA, 2013, p.483).

Referred to as the 'psychiatric bible' (Kutchins \& Kirk, 1993) for clinicians globally, the DSM-V offers a formal system for categorising and naming psychological difficulties (Marecek \& Hare-Hustin, 2009). Mainly informed by a modernist positivist epistemology in its assumption that only observable evidence derived from scientific methods can make knowledgeable claims, it has been criticised for pathologising human suffering and experience (Frances, 2013). It also challenges the very humanistic values embedded within the field of counselling psychology. Instead of working with an individual's specific needs and presentation, this medical diagnosis pathologises the clients using the same list of symptoms, rather than focusing on the clients presenting needs. Treatment also espouses the positivistic ideas under which the psychiatric model thrives, that is, a client presents with a diagnosis and their symptoms can be fixed. 
Helpful and unhelpful processes in psychological therapy for female substance users

This predominant model identifies addiction as a disorder that can be treated as a chronic disease involving biomedical and physiological symptoms. This definition reduces SUD to binary categorical understanding and fails to address the complexity of this construct. Additionally, it does not acknowledge cognitive / affective factors or external influences such as economic, social and political factors that can significantly impact the individual (Kalant, 2010). The view that addiction is harmful to the public also means that addiction is dealt with in the UK by the criminal system, and yet this cause and effect is questionable. This reinforces the negative stereotype that labels addicts as a risk to society and in need of punishment. According to Larkin (2006), an emphasis needs to be placed on why people are addicted and the conditions that underpin their drug use, rather than finding reductive explanations to fit people into labels that promote a certain identity.

\subsection{Treatment Modalities}

As individuals with SUD are heterogeneous with regard to a number of clinically important features and domains of functioning, it means that pharmacological and psychosocial interventions are generally applied in the context of various treatment modalities. Evidence-based psychosocial treatments include cognitive-behavioural therapies (CBTs, e.g., relapse prevention, social skills training), motivational enhancement therapy (MET), behavioural therapies (e.g., community reinforcement, contingency management), 12-step facilitation (TSF), psychodynamic therapy/interpersonal therapy (IPT), self-help manuals, behavioural self- control, brief interventions, case management, and group, marital, and family therapies (APA, 2010).

West (2001) uses the terms 'theory' and 'model' interchangeably and lists 130 contemporary models of addiction, comprising five groups. The first group involves 
Helpful and unhelpful processes in psychological therapy for female substance users

theories that attempt to provide insight into the conceptualisation of addiction in terms of 'biological, social or psychological processes, or some combinations of these (West, 2001, p.3). This second group focuses upon explaining why particular stimuli have a high propensity to become a focus for addiction. The third group explores individual susceptibility to addiction (genetic heritability), with the fourth examining how environmental and social conditions can increase or decrease a person's vulnerability to addiction. The final group of theories explore recovery and relapse. This scope illustrates the complexity and diversity of defining addiction and how it can be regarded as an environmental, individual or behavioural issue. This differentiation emphasises the difficulty in integrating different theoretical methodologies and treatment approaches, and how the notion of addiction has become increasing accepted as a multifaceted phenomenon.

In exploring the effectiveness of different treatments, CBT as a treatment model for SUD has demonstrated efficacy as both a monotherapy and as part of combination treatment strategies, however, recovery rates are less than optimal and compliance with CBT treatment procedures by those clients with SUD are often poor (McHugh, 2010, O’Connor \& Stewart, 2010, Duta, 2008). Broadly defined to include both behavioural and cognitive behavioural interventions in this context, cognitive behavioural theory places its focus upon functional analysis, motivation to change and behavioural strategies to avoid triggers (Carroll et al, 2001). CBT has several distinct interventions that focus upon motivation, contingency management and relapse prevention, in contrast to psychodynamic interventions that work with the individual's defence mechanisms, drives and object relations (McHugh, 2010, Johnson, 1999). A comprehensive review of outcome studies and meta-analyses of effectiveness studies 
Helpful and unhelpful processes in psychological therapy for female substance users

of psychodynamic therapy for major categories of mental disorders, found little evidence to support its implementation for SUD (Fonagy, 2015).

Although empirical support for different interventions is promising, it is most often garnered through efficacy studies when the treatment is carried out under optimal conditions. Most SUD treatments occur in service provision settings under conditions far from optimal. Thus a limited body of effectiveness research has been conducted examining these treatments without the stringent controls afforded by efficacy trials (McHugh, 2010). Thus, from the research on CBT and psychodynamic therapy conducted to date, neither has shown greater effectiveness in outcome in scientific studies conducted for the treatment of SUD (Tolin, 2010).

It is also clear from the literature, that the diagnosis of addiction can impact the type of treatment administered. The difficulty encountered by counselling psychologists working within the addiction field is the expectation (due to treatment providers assessment requirements) to adopt the default position of viewing addiction as a mental health disorder, using the DSM-V category of symptoms to diagnose patients, instead of working with what the client presents in the initial session. It could be questioned whether the psychiatric language imposed on clients at this early stage is a contributing factor in why retention rates in psychological therapy for SUD clients are so low.

Addiction treatment providers often reference addiction as a 'disease' and thus espouse a 'medical model' position that implies users have limited control over their behaviour. This is most frequently positioned under the 12-step recovery program, Alcoholics Anonymous (AA). There are differing views as to whether the problem lies within the person or whether it is a symptom of environment and/or social factors. The 
Helpful and unhelpful processes in psychological therapy for female substance users

Diathesis-Stress model also attempts to explain behaviour as a predispositional vulnerability combined with stress from life experiences. The focus is on the interaction between vulnerability and stress and how it may increase susceptibility to psychopathology, subsequently creating sufficient conditions for the onset of SUD (Ingram \& Luxton, 2005, as cited in Hankin, 2015). The consequences attached to the 'disease concept' and the 'Diathesis-Stress model' is that service users may be resistant to change, because there is nothing they can do about their biology. This also questions whether psychological interventions could be effective for something biological when pharmacological drugs are proposed as the only solution.

Both the medical and diathesis stress models adopt the basic assumption that the individual has a genetic predisposition to addiction. This medical domain has been, and continues to be, highly influential in the formation of psychological practice. The main criticism of this framework is the lack of attention it pays to individual differences compromising the client's sense of determination to change (Matto, 2004). Larkin and Griffiths (2006) talk about dispensing with this top-down diagnostic system for understanding addiction and suggest focusing instead upon an explicit bottom-up approach that looks at the negative consequences experienced by the person involved as a way of focusing on 'what works' for them as individuals.

By contrast, the 'free-will' model sees substance use as an active attempt by the user to create meaning in his or her life. One author suggests that people use drugs because they want to stimulate their dopamine levels to activate their brain's reward system (Tom \& Sellman, 2009). This philosophy conflicts with the dominant medical paradigm. The free-will model was developed by Becker and Murphy (1988) and has been developed further by Gruben and Koszegi (2001) and it presupposes complete 
Helpful and unhelpful processes in psychological therapy for female substance users

alignment of choices and time-consistent preferences (Vale, 2009). In other words, there is nothing wrong with the consumer, and it is down to personal choice whether to use drugs or not. The biggest criticism of this model is that is does not believe in relapse (Waal, 1999), rather, it believes people can stop of their own accord and that those individuals who become 'addicted' do so because they experience a negative shock from their drug use (Vale, 2009). If the meaning of 'addiction' is conceptualized as behaviour determined by personal choice, then this also questions the utility of psychological therapy as it implies abstinence is down to an individual's decision rather than an intervention.

There are some commonalities between the 'disease' and 'free-will' models. Both agree that addiction is defined by compulsion, despite negative consequences and self-administration. The differentiation between the two is that the disease model treats addiction as a mental disorder (DSM-5, 2013). In contrast, the free will model believes addiction is a state negotiated through human desire and intent (Davies, 2000). The 12step treatment approach, associated with the disease model, promotes the concept of powerless within the individual and a need to surrender to a higher power. In contrast, the 'free-will' model advocates that an emphasis should be placed on the individual to take responsibility for their recovery (Schaler, 2000).

Some findings have shown that AA is a helpful and cost effective recovery resource for adults with SUD, illustrating the importance of this spiritual network and questioning the impact of traditional psychological therapies on their own (Moos \& Timko, 2008, Kelly et al, 2010, Kelly \& Yeterian, 2008, Humphreys \& Moos, 2001, 2007, Valliant, 2001). As most treatment services insist on clients attending AA or NA in conjunction with their psychological therapy, AA's benefits have been associated 
Helpful and unhelpful processes in psychological therapy for female substance users

with: 1) clients spending time with individuals who support their efforts towards sobriety and 2) clients increasing confidence to maintain abstinence in social situations over a longer period of time (McGreevey, 2011). It has also been found that rates of abstinence are about twice as high among those who attend AA and higher levels of attendance correlate with higher rates of abstinence (Kaskutas, 2009). However, research on the effectiveness of AA can also be seen as controversial and subject widely to divergent interpretations, with studies addressing specificity using statistical approaches resulting in contradictory findings (Kaskutas, 2009). The main criticism concerns the variability in adherence to core tenets from group to group. Since, it is non professional in design, quality control measures are minimal and there is no way to ensure that every group adheres consistently to all its principles (Mendola \& Gibson, 2016).

The Cochrane group and Project Match in the USA (NIAAA, 1989) both recommend that people attending AA should be informed on the lack of evidence of effectiveness of such a program (Kaskutas, 2009). The National Institute on Alcohol Abuse and Alcoholism (NIAAA) conducted project MATCH, the largest and most expensive clinical trial, to test whether outcomes could be improved by matching clients to one-to-one interventions, such as motivational interviewing, AA's twelve steps and CBT. Findings revealed that patient-treatment matching is not necessary in alcoholism treatment as the three approaches were equal in effectiveness (NIAAA, 1996). However, the results did show that greater participation in AA during the first three months of treatment equaled a greater recovery outcome (NIAAA, 1996). This reveals the importance of the social network for individuals and having others that understand their affliction as the main active ingredient in AA. 
Helpful and unhelpful processes in psychological therapy for female substance users

In addition to the dominant, psychiatric perspective there are other approaches available for counselling psychologists to consider. For example, Robert West (2006) developed his 'motivational theory of addiction' and sought to integrate conscious choice processes and non-conscious motivational systems by adding conscious decision-making to classical conditioning and instrumental learning processes. $\mathrm{He}$ summarised his argument using the acronym PRIME: Plans, Responses, Impulses, Motives and Evaluations (see box below). The theory attempts to explain human behaviour in terms of a multi-level motivational system in which 'higher' levels evolved later and can only influence behaviour through 'lower' levels. The lowest level involves generating responses. The next level involves generating potentially competing impulses and inhibitions. The third level involves generating motives (feelings of want or need attached to a mental image of something). The fourth level involves generating evaluations (beliefs about what is right or wrong, beneficial or harmful, pleasing or displeasing); and the fifth level involves generating plans (self- conscious intentions relating to future actions)' (West, 2009).

\section{A diagram showing PRIME: A Theory of Motivation (West, 2006)}

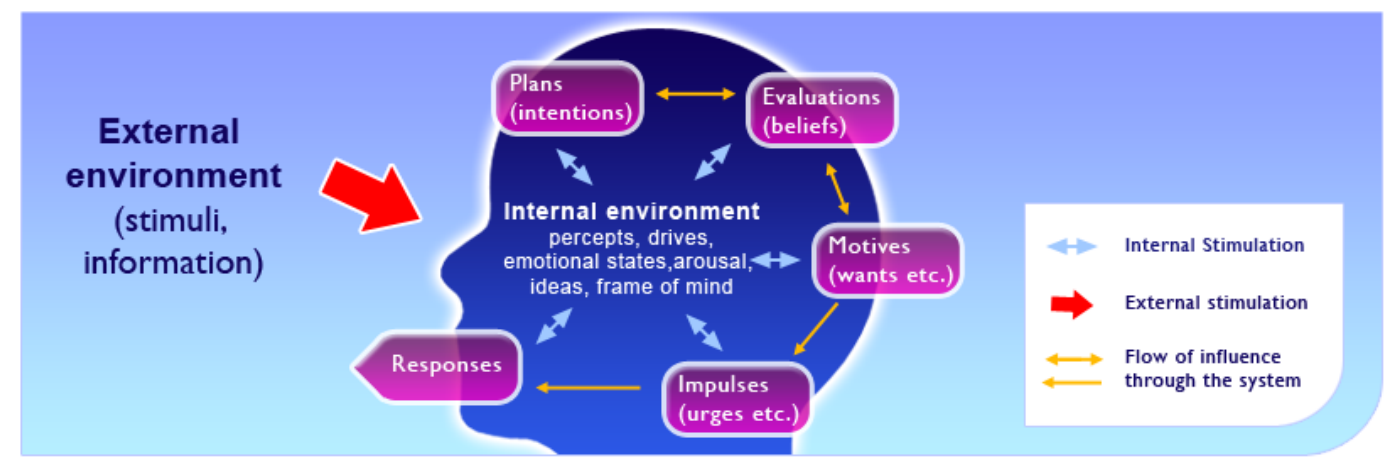

West argues that mindful forces are conscious feelings and beliefs that drive our 
Helpful and unhelpful processes in psychological therapy for female substance users

choices. Thus, addiction is seen as a behaviour that operates under partial operant learning mechanisms that are automatic, rather than a result of conscious choice. He contends that although most of the time people act on wants and needs without being actively self-aware, as much behaviour is driven by habit or instinct, 'occasionally we are led to be self-conscious and this has the capacity profoundly to influence our behaviour. This is the starting point for deliberate behaviour change' (West, 2009).

In his book, Theory of Addiction, West is critical of the medical model and its implication that addiction is an inherent biological condition. Equally, he is critical of the 'free will' model's position that addicted individuals use substances through choice. As a model that places itself somewhere in between the two, this motivational theory describes addiction as a learned response to reward seeking behaviour. However, his theory differentiates from other motivational theories, believing that these urges exist only in certain circumstances rather than operating on a continued existence (West, 2006). Consequently, the responses the individual self-consciously makes are within a cost benefit analysis that leads to the motivation to change.

From the different models described, it is clear that there is no clear definition that illustrates the complexity of 'addiction.' Despite the choice of approaches described, successful treatment outcomes (meaning completion of sessions and achieving abstinence) in SUD treatment is low, reinforcing the need for alternative exploration to discover what clients themselves find helpful. George Vaillant, an American Psychiatrist who has dedicated his life to understanding SUD, highlights what he feels most clinicians are missing when working with this client group:

A great deal of what people do is defensive and interferes with them seeing reality. If you can see beneath the surface, you see a great deal about what is 
Helpful and unhelpful processes in psychological therapy for female substance users

hugely relevant but invisible to most doctors. (George Vaillant, 2005 p.7).

In his paper 'A conversation with George Vaillant', he states that most doctors see unsuccessful clients with SUD presenting again and again for treatment. It could be argued that this citation illustrates the need to look beyond a client's diagnosis of being an addict and for clinicians to go into further depth about the underlying issues and behaviours. Often clients that successfully complete treatment do not return, thus the question of how they reached their positive outcome is rarely explored. Instead, the clients who fail to overcome their addiction occupy the minds of the clinicians. This could lead to a lack of motivation to work with this client group. In his longitudinal study, Vaillant also illustrates the importance of a client's life story as a predictor of recovery and relapse and believes that the instillation of 'hope' and 'meaning making' as important catalysts to change (Vaillant, 2005). He illustrates the need for clinicians to look beyond the definitions and therapeutic models of addiction and focus intrinsically on the causal factors personal to that individual. This thesis aims to adhere to Vaillant's suggestion to look beyond definitions and treatment models. As such, this thesis places a focus on how the women attach personal meaning to their psychological process of SUD treatment and the helpful and unhelpful aspects that enabled them to reach and sustain a position of recovery.

\subsection{Women and addiction}

It is time to recognise the fact that because of drug use many girls and women have been robbed of their identity, their dignity, their quality of life, and their right to protection. It is time to review our development paradigm and strategies, as well as our approaches to the drug issue, in order to make a real change and 
Helpful and unhelpful processes in psychological therapy for female substance users

one that will enable women who are affected by the drug problem to reclaim their humanity and their rights. (Duongsaa, 1998, p. 1)

Of the volumes of research on alcohol and drug addiction and recovery, there is a relative paucity of knowledge about women in particular and gender effects in general. Much research has proceeded under the unexamined assumption that gender was not an important experimental variable. This research focuses on women as studies, trials and statistics demonstrate that SUD is an area where the voices of women remain unrepresented in literature (Arfklen et al, 2001, Mallicoat, 2013). Treatment is often tailored around the male narrative, where truth is observed, known, named, and labelled in a masculine framework (SAMHSA, 2009). This has led to traditional SUD treatment promoting a gender bias, where the male perspective is the norm and the female perspective is excluded (Back et al., 2007; Brodsky \& Hare-Mustin, 1980; Niv, 2006; SAMHSA, 2010).

Most treatment models have been designed for men, based on male norms, ultimately suppressing women (Back et al., 2007; Bloom, Owen, \& Covington, 2004; Covington, 2008; Greenfield \& Grella, 2009; Grella, 2008). Traditional SUD programs have been designed to confront the addict about their abuse and resulting consequences (Tuchman, 2010). As women are more likely to experience high levels of shame and guilt in acknowledging their SUD within psychological therapy, confrontational approaches that serve to enhance these emotions have been found to be ineffective with female clients (Nelson-Zlupko, 1995, Tuchman, 2010). Another consequence of SUD treatment being accessed by a greater number of men than women, has led to research conducted predominantly using male participants (Tuchman, 2010). Hence the 
Helpful and unhelpful processes in psychological therapy for female substance users

evidence indicates that further research on women is necessary, with a particular focus on what aspects of psychological therapy is helpful or unhelpful for this client group.

The prevalence of SUD among women has dramatically increased in the past two decades (NIDA, 2016) and research has shown that women attend fewer treatment sessions than men (McCaul et al, 2001) and more likely than men to abandon treatment (Arfken, 2001). Current statistics show that women have a propensity to be the minority in most treatment settings despite the increase in numbers needing access to psychological support (Substance Abuse and Mental Health Services Administration, 2010; 2011; 2012).

Demographic and clinical characteristics that differentiate women from men with regard to SUD include greater psychiatric severity (longer episodes) and distress (Back et al., 2011; Grella \& Lovinger, 2012; Hernandez-Avila et al, 2004) as well as more issues with employment, medical problems and social impairments (Back et al., 2011). The literature also places women at a higher risk of relapse due to low selfefficacy, negative emotion and poorer coping skills than men (Connors \& Maisto, 2006; El- Sheikh \& Bashir, 2004; Moos \& Moos, 2006; Walitzer \& Dearing, 2006; Walton et al., 2003).

An exploration of existing research on female substance users found the literature placed an emphasis on causal factors, as well as the emotions that emerge from addiction (Kandall, 2010). Childhood experiences, damaged relationships (through foster care, abandonment, physical or emotional abuse) and victimisation (through prostitution, or homelessness) have been identified as causal factors in using drugs (Tuchman, 2010). Research also suggests that for women, a traumatic incident (e.g. childhood abuse) is significantly more likely than men to have happened prior to 
Helpful and unhelpful processes in psychological therapy for female substance users

the inception of substance addiction (Oumette et al, 2008). Conversely, new relationships or new responsibilities were identified as motivational factors to change (Briggs \& Pepperell, 2009). Emotionally, women feel more guilt, shame and anxiety about their addictive behaviours than men (NIMH, 2009). Therefore, these feelings may manifest in the therapeutic relationship and need active attention throughout the sessions. Despite this finding being useful for clinicians, it may also be a factor in why so many women are unable to complete psychological treatment. Research has also shown that women who have experienced physical and sexual trauma followed by posttraumatic stress disorder (PTSD), are more likely to have a diagnosis of SUD than men, highlighting the vulnerability of this client group and the depth of their despair when they enter treatment (NIDA, 2012). As women remain under represented in SUD treatment programs, new studies on treatment effectiveness and what they find helpful or unhelpful in psychological therapy is needed to assess gender differences in response to different treatment strategies.

In 2005, the National Treatment Agency (NTA) commissioned a study on gender differences in drug treatment to discover why the percentages of women and men who access support for SUD differs. Their conclusion found that drug use was more prevalent in men than women, which reflected the three-to-one disparity between male and female clients (NTA, 2010). The finding received criticism, as the monitoring data was in its infancy, however, recent statistics show that women presenting for substance use treatment represent $30 \%$ of the total number and this is increasing (NTA, 2012).

Drug services have attempted to address the treatment barriers facing women, such as sexual and physical abuse, childcare, and prostitution, by introducing women 
Helpful and unhelpful processes in psychological therapy for female substance users

only treatment sessions, accessible childcare and maternity services (NTA, 2010). Despite this positive step for women, drug workers have reported that fewer women seek treatment and do so in smaller number, especially parents concerned that their children will be taken into care (NTA, 2010). As women face such significant barriers to access treatment, it highlights the importance of retention and ensuring they remain in psychological therapy. Hence, the justification for this qualitative research examining the perspectives of women that have completed SUD treatment successfully.

In order for counselling psychologists to understand this client group more effectively, they need to gain insight into which elements women who have completed treatment successfully found most helpful. Instead of exploring the diagnosis and outcomes, the actual process of treatment from a woman's perspective needs to be understood, as this may hold the key to the factors motivating women to change and retain their abstinence post treatment.

\section{CHAPTER TWO:}

\section{Literature Review}

\subsection{Existing research in the field}

This chapter identifies the existing literature related to the research question and will address the aims and potential contribution of this study. A review of qualitative papers on service users perspectives of SUD treatment revealed a deficit across all journals, internationally and within the UK. Only a small fraction of papers addressed this issue, and no studies were identified that focused solely on the narratives of women 
Helpful and unhelpful processes in psychological therapy for female substance users

in this context. This deficit highlighted an unexplored area of research that deserves attention, and provides grounding for the present study.

\subsection{Quantitative v Qualitative}

In 2005, the journal Addiction published a review of qualitative methods in addictions research and noted only three qualitative research papers had been published the previous year (Neale, Allen \& Coombes, 2005). The low figures were attributed to the lack of confidence in scientific validity of qualitative methods, and a review of qualitative research published in Drug and Alcohol Review in 2015, found the situation remained unchanged. Addiction science remains dominated by biomedical and evidence based psychological practices (Miller et al., 2010) and currently, there is no journal dedicated solely to qualitative research in the field of alcohol and drug addiction with quantitative research remaining the dominant paradigm.

For this research, a review of the literature was conducted on a number of electronic databases in the UK and internationally. These included PyscInfo, ScienceDirect, PubMed and a NICE evidence search. Nursing and Social Science databases were also utilised. Findings revealed that the journals with the highest proportion of qualitative research on addiction were the following three journals: International Journal of Drug Policy; Drugs: Education, Prevention and Policy and Journal of Psychoactive Drugs. Journals with the lowest proportion of qualitative research studies of addiction included, the Psychology Of Addictive Behaviours, Addictive Behaviours, Addiction and Drug and Alcohol Dependence. In both cases, the number of qualitative research studies published was much lower than quantitative studies. 
Helpful and unhelpful processes in psychological therapy for female substance users

When conducting my own analysis using the database, PsycInfo (2016/17) using the search terms 'psychological treatment,' 'women' and 'substance use disorder' it yielded only 40 studies, two of which were qualitative studies. These were published in The Journal of Pain Medicine and Addictive Behaviours; neither examined female experiences of psychological treatment for SUD. Even though this analysis was conducted on a smaller scale, it supports the argument that this type of research is being neglected in both the journals and databases across the different health domains.

Broadening the original research criteria to include studies looking specifically at 'client perceptions of therapy' (regardless of client gender) yielded a number of studies including: clients' views of their therapist and the therapeutic relationship (Goldman et al., 2016, Manne, 2012, Golden \& Robbins, 1990, Rennie, 1992, Paulson et al, 2006); comparisons of the different approaches to therapy (Parry, Shapiro \& Firth, 1986); clients' views of treatment at various points of therapy (Roberts, 1993; Shilts \& Knapik-Esposito, 1993) and significant moments within sessions (Elliot \& Shapiro, 1992). However, these studies did not yield any results on the elements men or women perceived to be helpful and unhelpful in psychological therapy for SUD.

\subsection{Relevant Findings}

\section{- Helpful and Unhelpful Factors}

The literature review found only one published study specifically focused on the accounts of substance users who found psychotherapy helpful in assisting their recovery (Waters et al, 2014). Most research into psychological therapy has concentrated on the comparative effectiveness of different forms of therapy or the extent different theoretical formulations can account for the process of change, excluding subjective data. This narrative analysis study placed a focus on the role of 
Helpful and unhelpful processes in psychological therapy for female substance users

psychologists within this discourse. It explored the recovery narratives of women and men who were about to complete their 12-month psychological therapy under a clinical psychologist. The participants were all of white ethnicity and six out of seven were of English descent. To be included, all had to have experienced therapy as helpful in achieving abstinence or reducing their substance misuse.

The findings show that the relationship between the therapists and the clients developed as a result of the psychologists serving as secure attachment figures, and providing closeness, proximity, a safe haven and a secure base for clients. This is the only study identified that addressed what substance users identified as helpful. However, it differs from the current research for a number of reasons. Participants in the Waters et al., (2014) study were a mixed gender sample, all the same ethnicity (white) and over 40 years old and a narrative analysis. In addition, one of the main criticisms of this study relates to how a narrow focus is placed upon attachment, with the implication that this was the only prerequisite for a successful recovery. In interviewing women about their lived experience of psychological therapy, I propose to reach a broader sample of women in terms of age and ethnicity and will focus upon the process of psychological therapy. In addition, instead of working solely with psychologists, my participants will have experienced a variation of therapists engaged in different therapeutic models.

In 2012, NIDA published a guide to working with SUD clients and promoted techniques and aims conceptualising what they believed to be helpful. These included: enhancing a clients motivation to change, teaching a client adaptive coping and problem solving skills to maintain abstinence, and supporting clients with setbacks that might lead to relapse (NIDA, 2012). It also encouraged the development of a strong 
Helpful and unhelpful processes in psychological therapy for female substance users

therapeutic alliance and an empathic approach, paying attention to an individual's specific needs. Reyre et al (2017) also supported the idea of a strong therapeutic relationship as helpful. They found that professionals working in addiction treatment needed to place a greater emphasis on their own self-care through specific training to enable them to deal with higher levels of psychological distress, thus improving the engagement and trust within the therapeutic relationship. In terms of unhelpful, aggressive or confrontational approaches were found to have a negative impact (NIDA, 2012). Even though these findings are useful in terms of guidance for clinicians, they were proposed from the perspective of clinicians as a guide for other professionals, rather than incorporating client input.

When conducting the literature review, placing a particular focus upon helpful and unhelpful factors for SUD clients, research on 'substance users' perspectives on helpful and unhelpful confrontation: implications for recovery' emerged (Polcin et al, 2012). This American research differed from the British, Waters et al study, and the current thesis, as findings were not limited to psychological therapy but inclusive of interactions with family, friends, treatment staff and their workplace. Qualitative interviews were conducted on 38 substance users living in recovery houses, with $82 \%$ male and $79 \%$ white ethnicity. The data gathered was entered into a software system for coding that identified commonalities and differences. Participants were solely asked to recall and describe an experience of helpful and unhelpful confrontation while using drugs and when abstinent.

Findings revealed that contextual findings influenced how confrontation was experienced, such as timing, the confronter's confrontational style and the impact of important relationships (Polcin et al, 2012). Helpful confrontation was experienced 
Helpful and unhelpful processes in psychological therapy for female substance users

most in early recovery, especially if comments were from someone with knowledge about addiction. Unhelpful confrontation was when comments were perceived as too directive and intrusive. This study is insightful as it could be applied to how therapists work with confrontation within a psychological framework and whether confrontation can be perceived as helpful if the timing and style is appropriate. This differs from the current study as it focused solely on white male participants, focused upon confrontation and used a broader scope of interactions in the analysis rather than examining the process of psychological therapy.

Due to the limited findings on helpful and unhelpful studies within the context of SUD, the literature review was broadened to explore helpful and unhelpful factors in therapy. Elliot's (1985) significant events research investigated client identified important moments in therapy. The rationale was the presupposition that moments identified as having helpful impacts could be seen as a turning point within the therapeutic process. The results found: a new perspective, problem solution, problem clarification, awareness, understanding, client involvement, reassurance and personal contact as the 'significant events' deemed helpful by clients (Elliot, 1985).

Elliot's work was included in Timuluk's 2007 meta-analysis on qualitative studies that focused on client-identified impacts of helpful events in psychotherapy. The findings revealed only seven qualitative studies between 1985 and 2007, and not one focused on SUD clients. This again reinforces the need for current research into what is helpful and unhelpful for this client group.

Representing a small fraction of qualitative research within this field, Hodge \& Leitz (2014) explored therapist and client perceptions of using spiritually modified CBT for SUD treatment. This study placed therapists and clients in separate focus 
Helpful and unhelpful processes in psychological therapy for female substance users

groups and results found helpful aspects included; social support, divine coping resources and spiritual motivation. The potential challenges included therapists inadvertently imposing their own religious beliefs on clients and the possibility of offending clients, if they did have conflicting beliefs. The main criticism of this study was the lack of focus on the individual as it was conducted in a group setting. The approach also appeared to be more aligned with the AA spiritual philosophy than CBT and provide no insight into how these factors were helpful. This illustrates the need for further qualitative research that places an emphasis on the phenomenological experience of women who have completed psychological treatment.

As co-occurring mental disorders are prevalent in over half patients with addiction, this could also be a factor in low treatment success for SUD (EMCDDA, 2015). Patients that have dual diagnosis often exhibit symptoms that are more severe, persistent and resistant to treatment, and need medication and behavioural therapies to overcome their comorbid conditions (NIDA, 2012). It has been found that the identification of psychiatric comorbidity in substance users is problematic, because the acute or chronic effects of substance use disorder can mimic the symptoms of many other disorders (EMCDDA, 2015). Hence the poor outcomes in affected subjects. Ness et al. (2014) reviewed the literature that assessed the barriers in dual recovery treatment and found that the differentiation in treatment facilities (i.e. drug treatment services and mental health centers) was the greatest limitation to success. Services were found to lack sufficient expertise to treat both types of disorders (Sacks et al, 2013). The integration of a shared treatment plan, working on each disorder concurrently and the detection and assessment of comorbid substance use and mental disorders through validated instruments is highly recommended (DOH, 2017). 
Helpful and unhelpful processes in psychological therapy for female substance users

In 2017 UK Mental Health Services (DOH) commissioned a report (Drug Misuse and Dependence: UK guidelines on clinical management) that explored the therapeutic strategies for working with clients with dual diagnosis. The following strategies were identified as helpful: the strength of the therapeutic alliance, which was associated with positive counselling outcomes, a focus on retention in treatment, treatments that developed the clients self worth and multifaceted treatment to increase efficacy. In this study, the women were not asked whether they had a diagnosis of another mental disorder as the focus was placed specifically on psychological therapy for SUD and what they found helpful or unhelpful.

\section{- Summary}

The findings of the review on helpful and unhelpful factors reflect how few IPA studies feature in the field of addiction. It could be reasoned that this is because an existential phenomenological perspective is at odds with the diagnostic and treatment model of addiction (Larkin and Griffiths, 2002; Back and Greenfield, 2009; Stewart, Gavric and Collins, 2009; Shinebourne, 2010). Previous studies have focused upon quantitative methods as they fit within the medical paradigm that addiction treatment models have been tailored to fit. In measuring attributes of SUD, these studies have lacked the essence of the phenomenon of the individuals that experienced SUD and the meaning they ascribed to their experience (Covington, 2000, Bradley \& Ashley, 2005; Milligen et al, 2010; Merrick et al, 2012). Hence this research aims to fill the gap.

\section{- Concept of recovery}

Exploration beyond helpful and unhelpful within the addiction literature revealed the importance of the concept of 'recovery' and the meaning attached. As it features as an inclusion criteria in this study it has been included in this review. Efforts 
Helpful and unhelpful processes in psychological therapy for female substance users

to measure recovery are challenged by the lack of professional and cultural consensus on the definition and measurement of key constructs (recovery, remission, abstinence) (White, 2012), yet, having this concept is seen by clients as the ultimate goal within therapy (Laudet, 2008, White, 2012, SAMHSA, 2015). Laudet's (2008) paper asked the question 'what does recovery mean to persons engaged in that process?' and found that participants viewed 'recovery' as an ongoing process rather than an end point. As $86.5 \%$ of her participant's defined 'recovery' as total abstinence, this could have alluded to the pervasive influence of abstinence-based 12-step recovery principles on treatment practices. Even though this is insightful for therapists working with this client group, it highlights the importance of knowing how to reach that point in therapy.

There continues to be little agreement on how long a state of improved health must be maintained before recovery status can be assumed to be stable (White, 2017). Only consensus that factors shown to improve recovery, include: increased quality of life, improved self-efficacy, employment and spirituality (Worley, 2017). In this study, the women had to have been in 'recovery' for a year minimum to be included in the research.

\section{- Identity}

A further review of qualitative studies in the literature highlighted the importance of identity and its impact on relapse rates (Taite, 2014, Buckingham et al., 2015). To investigate how people can empower themselves within their recovery, London Southbank University explored the addiction-related identities of 61 people in AA and Narcotics Anonymous meetings to see how strongly they identified as 'addicts' and 'addicts in recovery' (Taite, 2014). Their findings showed that in terms of relapse, it didn't matter how people saw their own identity. However, there was a difference in 
Helpful and unhelpful processes in psychological therapy for female substance users

relapse rates based on how much difference people saw between the two identities. Those that preferred the identity of the 'recovering addict' experienced fewer cravings, lower relapse rates and felt more in control (Scharff, 2014). This highlights how a client's perception of a certain identity can be a crucial motivating factor to sustaining their recovery. In relation to the current thesis, this study provides insight into the importance of clients' perceptions of themselves and how these perceptions can impact a successful outcome. It also highlights the lack of research exploring how people with SUD manifest a shift in their identify from an 'addict' to that of 'addict in recovery' and what they found helpful in treatment to reach that place.

Of significance within the qualitative / identity related field is Smith and Rodriguez's (2014) study on young men's experiences of early recovery. It placed a focus on change and identity transformation within the group of narcotics anonymous (NA). Using Interpretive Phenomenological Analysis (IPA), they asked males who were one year into their recovery to talk about their shift to abstinence. The findings showed that the participants felt conflict in relation to their new sense of self and identity as they felt loss and disorientation within their relational world. However, this paper didn't explain how to engage with this loss and disengagement within treatment. Recognising they were abandoning an identity that failed them, participants in this study described their struggle to embrace a new identity congruent with their new social world. Even though this study highlighted the nuances and complexities inherent in participants' recovery journeys, it differed from the current study as the focus was not on the men's experience of psychological therapy, but how they constructed meaning to their new life. It also only sampled men and subsequently reinforced the gender bias in the current literature. 
Helpful and unhelpful processes in psychological therapy for female substance users

\subsection{Overview of aims and the potential contribution of this study}

In this chapter, I have discussed some of the challenges of the addiction and recovery construct, explored helpful and unhelpful studies and highlighted how the current drug and alcohol treatment system in the UK seems to be failing those seeking support. Additionally, I have argued why a qualitative study into women with SUD who have successfully completed psychological therapy should be investigated. There is therefore, a need for research that can inform practice and help practitioners improve the work they do, rather than relying solely on existing health policies on SUD treatment that are informed by a medical approach, and lack input from substance users.

The importance of gaining an understanding into what women found helpful and unhelpful is consistent with the humanistic values of the counselling psychology field and a user-centred agenda. Placing a client's perspective at the heart of therapeutic learning and research can open the minds of practitioners and counselling psychologists working with this complex client group. The existing research has shown that considerable emphasis has been placed on symptoms and achieving abstinence. However, the high relapse rates indicate a lack of understanding about how to support this client group in abstaining in the long term, and working through the issues that led them to develop a SUD. There also appears to be no literature on how to improve practices within the psychological therapy in terms of process rather, than outcome.

From the literature review, it is clear that practitioners do not have enough knowledge about the subjective experience of psychological therapy for drug and alcohol addiction from the perspective of the client. My aim is fill this deficit by discovering what aspects were deemed most helpful and unhelpful in reaching a place of abstinence. This insight will help practitioners tailor treatment to work with the factors identified as helpful. Although the proposed number of participants for this 
Helpful and unhelpful processes in psychological therapy for female substance users

thesis is small, it aims to contribute to current literature through high quality and rigorous qualitative analysis.

Recognising that the issue of how addiction should be conceptualised goes far beyond the scope of this research, my aim is to conduct an in-depth qualitative study to discover how eight women who completed therapy successfully and remain abstinent, experienced their psychological treatment. Using qualitative research will provide depth to their lived experience and represent their own meaning making experience. Qualitative research has placed increased emphasis on the complex interactions between environment, social and psychological influences on a person's behaviour, but the large scale survey format that has identified some of these relationships, may have missed examination of the processes by which social structural and psychosocial factors are mediated at the level of individual experience (McKeganey, 1995).

In highlighting the importance of acknowledging the multiple perspectives predominant in this field, this research aims to maintain a stance that appreciates the diversity of individual, subjective experience. In addition, this research also aims to provide something new to the literature while maintaining the ethos and philosophy of being a counselling psychologist. It is intended that this thesis will encourage further qualitative research into women with SUD and inspire practitioners to think critically about the current medical model and how they choose to work with this client group. 
Helpful and unhelpful processes in psychological therapy for female substance users

\section{CHAPTER THREE:}

\section{Method}

\subsection{Chapter Overview}

In this chapter, the methodological basis of the study is explained and a detailed account of the research method is given. The chapter is divided into three parts. The first, 3.0: 'Methodology', will review the rationale for this choice of method, the theoretical underpinnings of the research method chosen and the methodological procedure. The second, 3.6 'Research process', offers a detailed account of the research techniques used and how they were employed for this study. The third, 3.8 'Personal context and reflexivity', includes the researcher's reflections on the methodology chosen, as well as on personal experiences that are of relevance to the study. Several sections of the method chapter are written in the first person, in order to facilitate the reader's engagement with the process as it happened chronologically.

\subsection{Methodology}

\subsection{Research Question}

The research question is 'What are female substance users experiences of helpful and unhelpful processes in psychological therapy?' The population of specific focus is women diagnosed with substance use disorder that have successfully completed one to one psychological treatment. Success defined as meeting the criteria of attending and completing the therapeutic requirements set by the treatment provider. In this study, total abstinence was mandatory.

\subsection{Rationale for using IPA}


Helpful and unhelpful processes in psychological therapy for female substance users

Interpretative phenomenological analysis (IPA) is a qualitative research method with philosophical underpinnings in phenomenology and hermeneutics (Eatough \& Smith, 2008). It diverges from mainstream psychology as it focuses solely on in depth qualitative analysis in contrast to traditional qualitative and quantitative methods. It is a methodology well suited to the current research question, which asks women diagnosed with SUD how they experience psychological therapy and looks at the meaning they ascribe to its helpful and unhelpful aspects, seeking to understand the internal process within the individual and the meaning it holds for them. Instead of looking at a cause-effect relationship or simply the outcome of psychological therapy, this study focuses on understanding the subjective experience of psychological therapy in terms of setbacks, what women felt allowed them to progress, and how it was for them as women to have had this psychological experience and be able to express it in their own terms.

When psychology was founded as a discipline, the importance of the subjectivist (introspection) and objectivist (measurements of behaviour) were equally valued in psychological research (Farr, 1996; Jones and Elcock, 2001). However, gradually introspection gave way to behaviourism and quantitative methods gained dominance (Leahy, 2000). As qualitative research became increasingly marginalised, mainstream psychology took a positivist, hypothetico-deductivist approach, attempting to generate causal relationships between variables and to develop theories (Willig, 2013).

Even though the natural science paradigm continues to dominate psychology, the 'turn to language' perspective has meant that more focus is placed on discourse and understanding linguistic meaning (Madill, Jordan, Shirley, 2000). Smith et al. (2009) 
Helpful and unhelpful processes in psychological therapy for female substance users

identified four main qualitative approaches, phenomenology, grounded theory, discourse analysis and narrative analysis. Each qualitative approach holds a different epistemological stance and are associated with different forms of analysis (Willig, 2013).

Thinking within Willig's (2013) framework, it was important for this study that a greater focus was placed on the experiential, rather than just text and language. In asking women to identify what they found helpful and unhelpful in psychological therapy, this study seeks to understand this internal process in the individual and the meaning it holds for them. The focus is in understanding how participants experience psychological therapy and how they make sense of this experience. IPA is about incorporating all of this and acknowledging that we cannot access an individual's experience directly. It is therefore down to the researcher to interpret the data yet maintain a reflective stance around their own cultural and historical beliefs. This does not mean that the analysis is removed from the participant's experience, rather the opposite. The process is systematic and rigorous, embracing the phenomena as it presents itself to interpretation by participant and researcher (Willig, 2008). This interpretation can include the 'empathic-descriptive' level in which the researcher tries to 'stand in the participant's shoes' or a 'critical-hermeneutic' level where the researcher builds a descriptive narrative that differs from the participants account, yet offers a deeper understanding of the participants experience (Larkin, Watts and Clifton, 2006).

The double hermeneutic approach was selected for this research, as the aim was to identify the aspects of psychological therapy that women being treated for SUD found helpful and unhelpful without imposing my own meaning to the phenomena and 
Helpful and unhelpful processes in psychological therapy for female substance users

without formulating it into a theory. The phenomenological approach of IPA is to interpret people's perspectives and perceptions and examine how they are related to their experience of the world around them (Forrester, 2013). It is also ideal to utilize in the present study as "it enables that experience to be expressed in it's own terms, rather than according to predefined category systems" (Smith et al., 2009 p.32).

\subsection{Alternative Qualitative Methodologies}

Grounded theory was not selected as a methodology for this study, as I wanted to focus on the microanalysis of an individual's experience. Both identify themes, focus on content and then make sense of the phenomena. However, grounded theory works on larger sample sizes and aims to formulate theory to explain identified concepts on a broader level (Smith et al, 2009). In this study, however, it was important to represent the voices of these particular women in-depth, especially as they represent a client group highly underrepresented in the literature (Ashley, 2003) and that required a small number of participants.

Neither was a discourse analysis used for the current study, as Discourse Analysis (DA) is more social constructionist in its theoretical position. In looking at how language is used it privileges the things people say, rather than the people using it, and therefore does not fit with this study's objectives (Forrester, 2013). Forrester (2013) argue knowledge is constructed through our social practices rather than already in existence and that discourse is fundamental to the way we live our lives and how we interact. The factors that contributed to rejecting this method for this particular study included, the preference for naturally occurring text and ideal data, and the inherent focus on language. In this research the focus is phenomenological and centres on how 
Helpful and unhelpful processes in psychological therapy for female substance users

the women experience aspects of their psychological therapy. A study using DA would focus on the way that participants construct reality through language. Even though words affiliated with addiction, such as 'recovery' or 'dependence' will be considered in this study, IPA was selected as it places an emphasis on the subjective meanings attached to the experiential accounts, rather than the actual words used.

Narrative Analysis (NA) integrates several aspects of phenomenological and discursive psychology (Smith et al., 2009). IPA and NA share many similarities, such as the focus on the participant and interviewer, and ensuring the accounts produced in interviews are client led and unobstructed. Although an interpretation of themes drawn from the interview does occur, the primary focus is in the structure of the narrative itself (Murray, 2003). In telling autobiographical stories about themselves, people create and re-create their identities. NA can help construct meaning making (Crossley, 2000) and connect to the way personal identities are formed (Murray, 2003). Smith and Sparkes (2006) observe that narrative researchers can adopt realist (phenomenology) or relativist (social context) positions in relation to the narratives studied. It is similar to IPA in a phenomenological aspect, but looks beyond the 'face value' of an account to incorporate wider social and psychological meanings (Larkin et al, 2006). This approach was rejected, as this study was not interested in how the participants life chronological led to their current state. The focus was to assess how participants viewed and made sense of their past experiences in much greater depth.

\subsection{Epistemological standpoint}

To identify the epistemological position underpinning a research approach, Willig (2013) suggests that the researcher asks the following three questions: 1) What kind of knowledge do I aim to create 2) What are the assumptions I make 3) How do I 
Helpful and unhelpful processes in psychological therapy for female substance users

conceptualise the role of the researcher.

In exploring the subjective experience of psychological therapy for women with SUD, the focus is to capture the experience itself and avoid generalisations or claims about cause and effect relationships. In relation to the first question, the knowledge that I aim to create is a phenomenological understanding of what the experience of psychological therapy is like for women with SUD, specifically what they perceive as helpful and unhelpful about the therapy process. As the researcher, I will aim to make use of my subjectivity through the double hermeneutic and through reflexivity, to provide a constructive account of their experience. I also acknowledge that my training as an addictions counsellor and personal experience of addiction, could be influential in my thought process. Conceptualising my role as a researcher, I also acknowledge that my role as a scientist-practitioner will be shaped by my past clinical experiences and training. Even though every attempt will be made to remain impartial, I have to acknowledge that the generation of knowledge in this thesis will, in some way, be intertwined with the women's accounts and my subjectivity.

The epistemological position that underpins my role as a counselling psychologist and my standpoint in this study is that of a social constructivist. The social constructivist position states that what we perceive and experience is not a direct and unmediated representation of the environment. Instead, knowledge is constructed socially, historically, culturally and linguistically. A phenomenological IPA stance is about capturing the essence of psychological therapy for women with SUD and social constructivism relates to how this experience was socially constructed. It fits with the field of counselling psychology as it places the person and their subjective experiences centrally. Personally, I do not believe that there is one way of viewing the world, as interpretation is constructed through our social and cultural beliefs and the language we 
Helpful and unhelpful processes in psychological therapy for female substance users

use. From a postmodern perspective, the quest for universal laws is replaced by an understanding that knowledge is contextualised (Kvale, 1996). Instead of trying to discover pre-existing meanings, the production of knowledge is mediated on the participants' understanding of the questions and the researchers' interpretation of events. As an interpretative phenomenologist the aim is to seek meaning in the experience by reflecting upon the data and establishing wider meanings within that framework.

\subsection{IPA Methodology and Philosophical Underpinnings}

The focus of IPA is to explore in detail how participants make sense of their personal and social world (Smith et al, 2009). It is also interested in how people make sense of their life experiences (Smith, Flowers \& Larkin, 2009). The essence of "an experience" being defined as something that stands out from the flow of everyday experiences that we all go through (Smith et al., 2009). It endorses social constructionism's claims that sociocultural and historical processes are central to how we experience and understand our lives, including the stories we tell about ourselves (Willig and Stainton-Rogers, 2013). Since its inception, Smith (1996) has argued for an approach to psychology that is both experiential and experimental. IPA requires the researcher to engage in the process of looking in detail how an individual reflects on, and makes sense, of their experience. Thus it connects phenomenology and hermeneutics, with an idiographic lens (Smith et al., 2009).

IPA also acknowledges the influence of symbolic interactionism with its concern for how meanings are constructed by individuals within both a social and personal world (Denzin, 1995). Interactionists believe that people are 'out there' in a 
Helpful and unhelpful processes in psychological therapy for female substance users

social world making their own understandings of that world, in relation to their understanding of other people and themselves and the interaction between the two (Howitt, 2010). IPA assumes a connection between what people say, their thinking, and their emotional state, and recognises that people are complex and may not always want to self-disclose. Interpreting a participant's mental and emotional state from what they say is important amongst women diagnosed with SUD due to their complex presenting issues. The strength of the interpretive actions of the researcher is vital to how the information gathered is reflected, interpreted and analysed (Conrad, 1987). In order to further understand what IPA is, its three main influences, phenomenology, hermeneutics and idiography, will be briefly discussed.

\subsubsection{Phenomenology}

In everyday life each of us is something of a phenomonologist insofar as we genuinely listen to the stories that people tell us and insofar as we pay attention to and reflect on our own perceptions (Halling, 2008 p.242).

Phenomenology is the study of human experience and the way in which things are perceived as they appear in consciousness to the individual (Langdridge, 2007). Husserl (1889-1938) was the pioneer of this line of enquiry and was particularly interested in how someone could transcend their 'natural attitude' to be able to view their experience in a phenomenological way (Smith, Flowers, Larkin, 2009). The core philosophical basis of Husserl's movement was a rejection of the presupposition that there is something behind, underlying or fundamental to experience, when actual experience is the point at which we start (Smith, 2008).

Husserl challenged the mind-body dualism that was prevalent until the $20^{\text {th }}$ Century, by calling for a focus on "the things themselves", the experimental content of 
Helpful and unhelpful processes in psychological therapy for female substance users

consciousness (Langdridge, 2008; Smith el al., 2009). Instead of taking for granted our everyday experiences, Husserl felt that we should disengage from our natural attitude and adopt a reflexive phenomenological stance (Langdridge, 2007). He used the term 'intentionality' to describe how every act is made up of the 'how' and 'what' in the way we experience it. The concept of lifeworld, the world as it is experienced by the individual, is the focus of phenomenological psychology. It describes how our world is the subjective experience of consciousness. It reinforces the view that any access to the subject's experience will be second hand and viewed through the experience of the researcher (Smith et al., 2009). How a person views the world is often embodied in a particular historical and social context. This phenomenological approach has the task of elucidating the 'taken-for-granted' assumptions by which people navigate their lifeworld (Smith, 2008). According to Smith (2008) individuals often take for granted the meanings they attach to experiences, and exploration can lead to revelations that they may never have discovered previously

Drawing upon Husserl's thinking, in engaging in a phenomenological attitude we can use the process of phenomenological reduction to bracket off and reflect on what therapy was like for the women from their experiential reality. The aim of bracketing (epoche) is for the researcher to avoid preconceptions and presuppositions that could impact how they understand their participant's experiences. It is acknowledged that bracketing is an ideal that can never be fully achieved. However, this line of inquiry does enable researchers to produce innovative research as it helps them to not just reproduce their own view of the world, but rather to explore new ones (Langdridge, 2008). Husserl believed phenomenology was concerned with the primary reality as it appears, itself the phenomena and that human experience is a system of interrelated meanings (Smith, 2008). 
Helpful and unhelpful processes in psychological therapy for female substance users

Although a number of diverse approaches have been developed within phenomenology, the focus of the subjective experience remains a fundamental principle. Sartre (1958), Merleau-Ponty (1962) and Heidegger (1927) also contributed to this philosophical perspective and developed phenomenology from the first person perspective. Critical of Husserl's transcendental quest, Heidegger proposed that the human being is a 'Dasein.' The literal translation of which is 'being there,' but is most often referred to as 'being in the world' (Spinelli, 1989). This differs from Husserl's idea of dualism that we exist in the world as subject / object or person / world (Willig and Stainton-Rogers, 2013). Heidegger marked a move towards a hermeneutic and existential view of phenomenology that is now an integral part of IPA (Smith et al., 2009). The view of the human being as always being in context relates to the concept of intersubjectivity (Smith et al., 2009). As individuals we can never escape from our own engagements and experiences that have shaped us as individuals. Intertwined with these experiences, we are always trying to make sense of the world and attempting to understand our own meaning.

The development of how we are embodied in the world and our search for meaning is the starting point of IPA (Eatough \& Smith, 2008). Merleau-Ponty developed the body-subject idea to illustrate that one's perception of the world is different to another person's experience, as it is felt through a different body (Smith et al., 2009). Sartre also contributed to this view that we are embedded in the world and in constant interaction with it through our relationship with others (Smith et al., 2009). His introduction of an existential lens shed light on how the process of interpretation is necessary to gaining an understanding of our self and others.

\subsubsection{Hermeneutics}


Helpful and unhelpful processes in psychological therapy for female substance users

This then leads to the process of hermeneutics and the theory of interpretation, which constitutes another major theoretical underpinning of IPA. It has been described as:

Something foreign, strange, separated in time, or experience, is made familiar, present, comprehensible, something requiring representation, explanation or translation is somehow 'brought to understanding' - is 'interpreted. (Palmer, 1969, p.13).

This strand of philosophy is concerned with the idea that every interpretation is contextualized in previous experience and through our social, historical and cultural context (Heideigger, 1962). It entertains the possibility of uncovering the intention of the original meanings of an author (Smith et al., 2009). Our understanding of an event or an object is always mediated by an already existing knowledge accrued from our experiences in the world (Smith, 2007). As we form interpretations based on our existing knowledge and beliefs, Heidegger suggests that priority should be given to the new object rather than to one's previous preconceptions (Smith, 2007).

Heideigger states that instead of 'working out the fore-structures in terms of the things themselves' we may only know 'our preconceptions once the interpretation is underway' (Heidegger, 1962, p.191). Additionally, it may not be possible to know in advance what preconceptions we hold. The interpretive element of hermeneutics is essential since links between the participant's experiences and the researcher's interpretation of those experiences are encouraged in order to enable the researcher to draw out meanings from the participant's accounts which otherwise might be difficult for that person to articulate (Smith, 2003). IPA involves a double hermeneutic (Smth et al., 2009), as the researcher is required to make sense of the participant, who is 
Helpful and unhelpful processes in psychological therapy for female substance users

making sense of the thing itself. This, and reflexivity, are integral parts of the methodology and how analysis is formulated.

\subsubsection{Idiography}

The third philosophical component of IPA is idiography, which refers to the concern of the particular. The humanistic psychologist, Allport (1940) noted that an idiographic approach to psychological research meant that each individual should be studied as a unique case (Smith, 2008). A focus on the particular was seen as more important than a nomothetic approach, which assumes that the behaviour of a particular person is the outcome of laws that apply to all (Smith et al, 2009). A detailed case study of the female participants offers opportunities to learn a great deal about each one as an individual person and their response to a specific situation, by focusing on the particular rather than the universal. IPA studies express their commitment to idiographic designs by their use of single case studies, before they attempt cross case analysis at within and between levels (Smith, 1991; Smith, Michie, Allanson and Elwy, 2000; Bramley and Eatough, 2005). The strength of using IPA is that attention to the individual can illuminate the shared commonality of human experience and that probing into a person's experience can take us nearer to the universal (Warnock, 1987). Idiography explicitly addresses the subjective and interpersonal involvement of human emotion, thought and action and the chaotic aspects of human life (Willig and Stainton-Rogers, 2013). Within the idiographic frame, the individual case could be seen as part of a wider study involving a number of participants. Thus the individual becomes a starting point in the process of analytic induction, by connecting the individual with other additional cases to develop a theory from the 'ground up'. 
Helpful and unhelpful processes in psychological therapy for female substance users

\subsection{Research Process}

\subsubsection{Participants}

This qualitative study aimed to recruit a sample of female substance users who had completed individual psychological therapy for their substance use disorder. The emphasis within IPA research is to gather a purposive, homogenous sample. This ensures that the phenomena being investigated is relevant to all participants included in the study (Bramley \& Eatough, 2005). IPA studies are conducted on small participant numbers as the analysis of individual transcripts prioritises depth of the perceptions and understandings of the client group, rather than making general claims (Smith, 2008). The number of IPA participants required for postgraduate study has been discussed across the IPA community (Smith et al, 2009; Reid, 2005). In a review of the IPA corpus (Smith, 2011), the number of participants ranged from 1-40, with a mean of 14 . However, this included studies with focus groups, which raised the mean. It has been suggested that there should be a maximum of ten participant's in an IPA study (Reid et al, 2005) as smaller numbers are regarded as preferable to encapsulate participants meaning of their experience (Smith \& Eatough, 2006).

Eight women were recruited for this study. All participants were over 18 years of age and spoke English as their first language. The mean age was 34 and seven participants identified as white British and one black British. Each woman had been given a diagnosis of substance use disorder under the DSM-V criteria and had completed successful one to one psychological treatment within the past year.

\subsubsection{Recruitment}


Helpful and unhelpful processes in psychological therapy for female substance users

Participants were recruited by approaching a number of addiction related charities across London. I anticipated that recruiting women for this study would be difficult due to the sensitivity and shame felt around this topic, however, this concern was unfounded and recruitment process was much quicker than I anticipated. Contact was made with the management of the various charities, and participants that fit the criteria for the study were recruited from two addiction charities, using a snowballing sampling technique. Snowballing refers to a method of selecting a sample in which participants are asked if they know of other potential participants with relevant characteristics who might be approached.

Considering that this client group is highly vulnerable, I asked for assistance from the charities into which women they deemed most suitable to participate, as they already had a relationship of trust and could act as intermediaries. Initial contact was made by email to each manager describing the study (Appendix 2) and enclosed was an information sheet that was used to supply any potential participants with details of the study (Appendix 3). After informing them of my research, I shared my inclusion / exclusion criteria and offered my contact details for any further details (Appendix 2). I exchanged contact details with interested participants, and we arranged to meet. Potential participants were then supplied with a consent form (Appendix 4) outlining their right to withdraw from the study at any time, and the principles of confidentiality. They were also given a demographic questionnaire (Appendix 5), an interview schedule (Appendix 6) and a participant de-brief form (Appendix 7). I anticipated that some of the participants might discuss their interest in the study with their previous counsellors before contacting me directly, and therefore, I requested that the charities informed the counsellors about the nature of the study. 
Helpful and unhelpful processes in psychological therapy for female substance users

Once the first participant had been interviewed, a snowballing effect proved the most effective recruitment tool. Names of potential participants were emailed across by the treatment manager or referred by women in the study. All participants had to fit the inclusion criteria and details were discussed with the respective treatment manager before participation was agreed. As I had expected this sample population to be difficult to recruit due to their vulnerable nature, this method meant that participants were found in a short space of time. However, a potential recruitment bias was the risk that the women may have discussed with each other questions in advance. To overcome this, they were asked not to disclose their own interview experience with other potential participants apart from what was written in the information form. There was also concern that the women felt privileged to be approached, and I wondered if they felt their responses needed to reflect their positive outcome in psychological therapy, thus recalling more helpful than unhelpful factors.

While potential participants expressed interest in having their voices heard, they were insistent that their identities would be anonymous. In particular, they felt strongly that they did not want their therapist to be able to identify them. I was very conscious that data collected would contain no identifiable information linked to that person and reflected on how this could impact on what information they shared. To compound their fears, confidentiality was confirmed, leading to the women being able to relax and begin to talk freely.

\subsubsection{Inclusion / Exclusion criteria}

The inclusion criteria for participants in this study included: 
Helpful and unhelpful processes in psychological therapy for female substance users

1. Women diagnosed with substance use disorder according to the DSM-V criteria, meeting the treatment providers assessment criteria.

2. Women that had completed three months of one to one psychological therapy for their substance use disorder meeting the requirement of the treatment provider.

3. Abstinence from drugs or alcohol, or reduced their intake, adhering to the policy of the treatment provider.

4. Engaged in a minimum of weekly individual psychological therapy for the duration of treatment.

The exclusion criteria were:

1. Women with a psychiatric diagnosis of psychosis from a mental health worker.

All the participants fit the inclusion criteria required. A number of the participants were interested in my motivation around completing this study and whether I was in recovery myself. Despite not being an addict, I explained that I had worked as an addiction counsellor and, had addiction within my family, but did not disclose about whether I was in recovery or not. I feared their responses could be biased if I divulged my motivation and background and may influence what they shared. Therefore, any questions were conducted post interview.

Table 1 below outlines the participants' basic demographics, the charity that they were recruited from, their drug of choice, the type of therapy they had received and how long they had been in recovery.

Table 1: Participant Demographics. 
Helpful and unhelpful processes in psychological therapy for female substance users

\begin{tabular}{|c|c|c|c|c|c|}
\hline Name* & $\begin{array}{l}\text { Drug of } \\
\text { Choice }\end{array}$ & $\begin{array}{l}\text { Treatment } \\
\text { Provider }\end{array}$ & $\begin{array}{l}\text { Therapy } \\
\text { Modality }\end{array}$ & $\begin{array}{l}\text { Ethnicity } \\
* \text { WB } \\
\text { White } \\
\text { British } \\
\text { *BB } \\
\text { Black } \\
\text { British }\end{array}$ & $\begin{array}{l}\text { Time in } \\
\text { Recovery }\end{array}$ \\
\hline Sam & $\begin{array}{l}\text { Heroin } \\
\text { Crack } \\
\text { Cannabis } \\
\text { Alcohol }\end{array}$ & $\begin{array}{l}\text { SERVICE } \\
\text { A }\end{array}$ & Integrative & WB & 12 months \\
\hline Anita & Alcohol & $\mathrm{A}$ & Psychodynamic & WB & 12 months \\
\hline Emma & $\begin{array}{l}\text { Heroin } \\
\text { Prescription } \\
\text { Alcohol }\end{array}$ & B & Integrative & $\mathrm{BB}$ & 15 months \\
\hline Kat & $\begin{array}{l}\text { Heroin } \\
\text { Cocaine } \\
\text { Alcohol }\end{array}$ & B & Integrative & WB & 13 months \\
\hline Jules & $\begin{array}{l}\text { Heroin } \\
\text { Alcohol }\end{array}$ & B & Psychodynamic & WB & 14 months \\
\hline Louise & $\begin{array}{l}\text { Heroin } \\
\text { Crack } \\
\text { Cannabis }\end{array}$ & $\mathrm{A}$ & $\begin{array}{l}\text { CBT / } \\
\text { psychodynamic }\end{array}$ & WB & 13 months \\
\hline Rachel & $\begin{array}{l}\text { Heroin } \\
\text { Crack } \\
\text { Alcohol }\end{array}$ & $\mathrm{A}$ & Gestalt & WB & 14 months \\
\hline Noreen & $\begin{array}{l}\text { Heroin } \\
\text { Alcohol }\end{array}$ & $\mathrm{A}$ & Psychodynamic & WB & 12 months \\
\hline
\end{tabular}

* All of the participants were given pseudonyms

\subsubsection{Data Collection}


Helpful and unhelpful processes in psychological therapy for female substance users

Semi-structured interviews were used to collect the data. All interviews were expected to last an hour. This was felt an appropriate length of time to ensure the women did not find the process too tiring. Semi-structured interviews are often considered the gold standard in data collection for IPA (Smith, 2009). Therefore, this qualitative approach was selected to gather data that would help identify the participants 'experiential claims' and 'objects of concern' and to develop a phenomenological account of their lived experience (Smith, Flowers, Larkin, 2009).

As the researcher, I wanted to engage in dialogue, be able to modify questions and delve deeper into areas that emerged. An interview schedule was developed to guide my inquiry that included prompts to ensure the conversation was as open and flowing as possible. The relative informality of following the participant's responses was intended to facilitate a sensitive and empathic account that reflected a human-tohuman relationship (Fontana and Frey, 2000).

The interview schedule was informed by discussions with my supervisor, and guidance on interview development sought through published guidelines (Smith \& Osborn, 2003). Having an interview schedule prepared kept the focus on the research question, and helped anticipate any problems as they arose. The schedule was used flexibly, in order to allow probing of unanticipated areas that emerged, allowing prompts such as (How did that make you feel? Can you tell me what that meant for you?). This encouraged further clarification from the participants to ensure that I understand their meaning.

The following questions were included in the interview schedule (Appendix 8).

1. What brought you into therapy? 
Helpful and unhelpful processes in psychological therapy for female substance users

2. What aspects of the therapy did you identify as helpful (if anything)?

2.1 Why was it you felt this was helpful?

3. Which aspects of the therapy did you identity as unhelpful (if anything)?

3.1 Why was it you felt this was unhelpful?

3.2 How did this leave you feeling?

The questions were open ended, as the purpose of the interview was to have a 'conversation with a purpose' (Smith, Flowers and Larkin, 2009). In starting the dialogue with the question; 'what brought you into therapy?' participants were facilitated to tell their own experience in their own words. To ensure they focused on their concerns, I avoided leading the questions and becoming over empathic, to prevent influencing their responses.

A pilot interview was conducted, a step generally supported by a number of researchers (Kvale, 1983). Conducting a pilot interview offered the opportunity to see how participants might respond to the questions, assess the time required for the interview, and to reflect on effectiveness of my approach. Listening to the recorded session assisted me to improve my interview technique, by improving my listening and allow more space for the participant to talk. As the pilot participant was a colleague with a history of addiction, they were not incorporated into the main volume of data, as this participant wanted her answers to remain anonymous. I invited feedback and any general comments from the participant regarding the questions. However, she did not raise any concerns, therefore no changes were made to the format of the interview schedule. 
Helpful and unhelpful processes in psychological therapy for female substance users

\subsubsection{Procedure}

Interviews were undertaken at the recruiting agencies from which the participants were drawn, to ensure that clients had support if any anxiety arose. They were conducted in counselling rooms and in the afternoon when the agencies were at their quietest. This gave privacy and enabled the interview to be conducted confidentially, with few distractions. Prior to the onset of the interviews, I gave the participants the information sheet detailing the study (appendix 3), a consent form (appendix 4) and a demographic questionnaire (appendix 5).

The consent form outlined the aims and purpose of the study and the participant's right to withdraw up to the point of analysis. The consent form also highlighted the limits of confidentiality, as the women were highly sensitive around being identified. I informed them that each person would be given a pseudonym and identification number to anonymise them. The interviews did not commence, and was not audio recorded, unless the participants had consented to take part both verbally and in writing. Furthermore, I did not proceed with any interview until the participant was feeling comfortable and had clearly stated that they were ready to begin.

The participants were informed that the data would be stored (securely, for a period of ten years before being destroyed). Post interview the participant was given a debrief form (appendix 7). This form contained several useful contact numbers for addiction support, and/or if they required additional support if they felt that anything from the interview triggered any risk to their recovery.

Interviews ranged from 45 minutes to 1 hour 10 minutes and informed prior to beginning that it could take longer. Each participant consented to their interview being audio recorded and offered the chance to view their transcripts at a later date, to see if 
Helpful and unhelpful processes in psychological therapy for female substance users

there was anything they wished to withdraw. Even though all women were given this opportunity, none of them followed this up.

\subsubsection{Ethical Considerations}

The research gained full ethical approval from The University of Roehampton Ethics Committee, 2015 (Appendix 7). It also adhered to the British Psychological Society's Code of Ethics and Conduct (2009). I was particularly concerned that confidentiality and the protection of the participants' anonymity were maintained at all times. This was an important ethical consideration given the sensitive and personal nature of the topic that was under investigation and the vulnerable nature of my sample. The agencies used for recruiting participants charity A and charity B granted me permission to access their clients before any participants were interviewed. The managers at both treatment services recommended women that they felt would be best suited for the study. I assessed the risk of the women feeling coerced into participating and ensured that they wanted to participate willingly. For those clients who were recommended to me through word of mouth, I consulted agency staff regarding their suitability for the study before any interviews were undertaken.

The process of interviewing meant adhering to the distinct boundaries between my role as a counsellor and researcher. I feel very strongly about supporting vulnerable women and I had to monitor whether I was empathising too strongly at times. I observed my own feelings to ensure that participants were able to focus on their own responses and not be distracted by mine. The use of a reflective journal was a useful tool for managing these feelings and allowed me to develop as a researcher as each interview progressed. 
Helpful and unhelpful processes in psychological therapy for female substance users

\subsubsection{Quality}

The issue of validity in any qualitative study is complex. Historically, the criteria for assessing the validity of quantitative research in psychology was to achieve scientific status modelled on principles derived from physics. In contrast, qualitative research places importance on social context and recognises the impossibility of achieving an entirely neutral and objective standpoint (Smith et al., 2009). Amid the rise of qualitative research in scientific communities, considerable debate has ensued regarding epistemological, philosophical and methodological issues (Whittmore et al., 2001). Validity standards are often seen as challenging because of the necessity to incorporate both rigor and subjectivity as well as creativity into the scientific process (Whitmore et al., 2001). Because qualitative research is based on entirely different epistemological and ontological assumptions it does feel inappropriate to evaluate it with positivistic criteria.

To manage this complexity, common criteria have been developed to enhance and assess validity in qualitative research (e.g. Taylor, 2001, cited in Howitt, 2010 and Yardley, 2000). Yardley's (2011) four principles for evaluating the validity of qualitative research include: sensitivity to context, commitment and rigour, coherence and transparency and impact and importance. The first principle, sensitivity to context was demonstrated by recognising interactional difficulties and negotiating the intricate power play between researcher and participant. Commitment to rigour referred to the thoroughness of the data in how it was collected, coherence and transparency related to the stages of analysis and how these were written. Finally, the validity of impact and importance lies in the findings and what it informs the reader. Yardley emphasises that although it is important that value is demonstrated, validity criteria should be seen as 
Helpful and unhelpful processes in psychological therapy for female substance users

highlighting quality issues rather than providing a rigid checklist that restricts the freedom and flexibility of researchers (Rouse, 2012). Validity remains an important ethical issue as it means that participants' contribution is valued through rigorous research. Hence, why Yardley's steps were followed in this study.

\section{- An independent audit}

An independent audit is one way to assess validity in qualitative research (Smith et al 2009). The principle is to keep a paper trail so that someone could in theory follow the process from initial proposal to final report. Thus, this thesis includes a file of material including the initial research proposal, the initial list of themes, and process that led to the final selection. To ensure validity, the data was sent to an external IPA supervisor to examine credibility. It was deemed transparent and coherent, adhering to Yardley's criteria of validity. The purpose of the audit is not to find an account, which is the one and only truth, but to ensure quality and transparency. The inclusion of this material enhances the transparency of the analysis and allows the reader to assess the consistency of my interpretations.

\subsubsection{Potential Distress}

There was a risk that taking part in this study may be potentially distressing to participants. This was addressed by providing substantial information about the nature of the research and the topics that would be covered before participation, so that potential participants could make informed decisions about taking part. Participants were made aware that they could ask for a break at any time and had the right not to answer particular questions if they did not want to. They were also informed that it was their right to withdraw from the study at any time, without having to justify their 
Helpful and unhelpful processes in psychological therapy for female substance users

decision. Having worked with this client group as part of my counselling psychology training, I endeavoured to conduct the interviews as sensitively as possible.

I kept the focus of each interview on helpful and unhelpful factors of psychological therapy, which some participants reflected back to me as a positive aspect of the interview. For some of the women talking about what had worked in therapy appeared to be a reminder of how to stay safe. Only one participant got emotional during the interview, and I made her aware that we could stop if she felt that it was too much. This participant reflected back to me at the end of her interview, that the interview was a 'cathartic experience' as her distress had been a reminder of how far she had progressed in her recovery, as well as expressing her fear of "ever going back to that dark place".

Post interview, each participant was given the space to reflect on their feelings and emotional state. I also checked with them what their plans were for the rest of the day and what support they had available. In going through the de-brief form, I ensured that they had the helpline numbers for the charity. All the participants also had access to a member of staff in the agency if they felt they needed further support. The majority of the women appeared to be positive about having their voice heard and having an opportunity to praise their therapists. A number of women expressed that, psychological therapy had saved their life and their gratitude was palpable.

I did not experience any distress during the research process. There were staff present in the treatment agencies and I would have terminated any interviews if I felt concerned.

\subsection{Data Analysis}


Helpful and unhelpful processes in psychological therapy for female substance users

The existing literature on analysis in IPA has not prescribed a single 'method' for working with data. However, it is characterised by the descriptive to interpretive process (Reid, Flowers \& Larkin, 2005). As a novice to IPA, prior to undertaking this study, I was aware that the analytic task was the most important. I therefore joined the London IPA group and attended a workshop to increase my understanding. I found the process of transcription time consuming and lengthy as each interview had to be transcribed from an audio recording into text. However, this did increase my familiarity with the data and I became immersed in the process. I anonymised the transcripts by removing any details about the charity from which participants received treatment, as well as any personal, identifiable information. Each interview was transcribed and analysed one at a time to ensure that the close, line by line analysis of the experiential claims, concerns and understandings of each participant was given the focus it deserved (Watts \& Clifton, 2006).

\subsubsection{Individual case analysis}

In keeping with IPA's idiographic approach, the data analysis began with reading and re-reading each transcript from start to finish. Each recording was listened to at least once to ensure that nothing was missed on the transcripts. Initial annotations were made in the right margin with exploratory comments describing initial thoughts about the content, language use and then conceptual, interrogative comments were added (Smith et al. 2009). This free coding is part of the process of being transparent and reflexive about what is brought into the analysis. Its intention is to allow engagement with the data from the participants' point of view, having noted the 'lenses' I might make sense of others ideas and prevent them from intruding on the subsequent process of idiographic coding and analysis. 
Helpful and unhelpful processes in psychological therapy for female substance users

A detailed line-by-line analysis was conducted to summarise and capture the essence of what each participant was trying to convey. This aim was to try to imagine what it is like to be the participant and to identify the main phenomenological ideas in the participant's words, remaining close to the participant's explicit meaning (Smith et al, 2009). The stages of coding included descriptive coding, linguistic coding and conceptual coding. Descriptive coding highlighted the main issues, key phrases and quotes, that I felt demonstrated what the participant was trying to convey. The linguistic stage focused on the participant's use of language. The pauses, the laughter and body language were also noted. The conceptual stage of coding was about engaging reflectively on each account to capture any meanings beyond the discourse. I had to retain my focus understanding what the participant had said and what it meant to them from the researcher's subjective experience. This is about moving away from the participant's explicit claims and drawing upon their view and meanings of the world. An example of my analysis is demonstrated in Figure 1 below and Appendix 8.

FIGURE 1: Example of Analysis 
Helpful and unhelpful processes in psychological therapy for female substance users

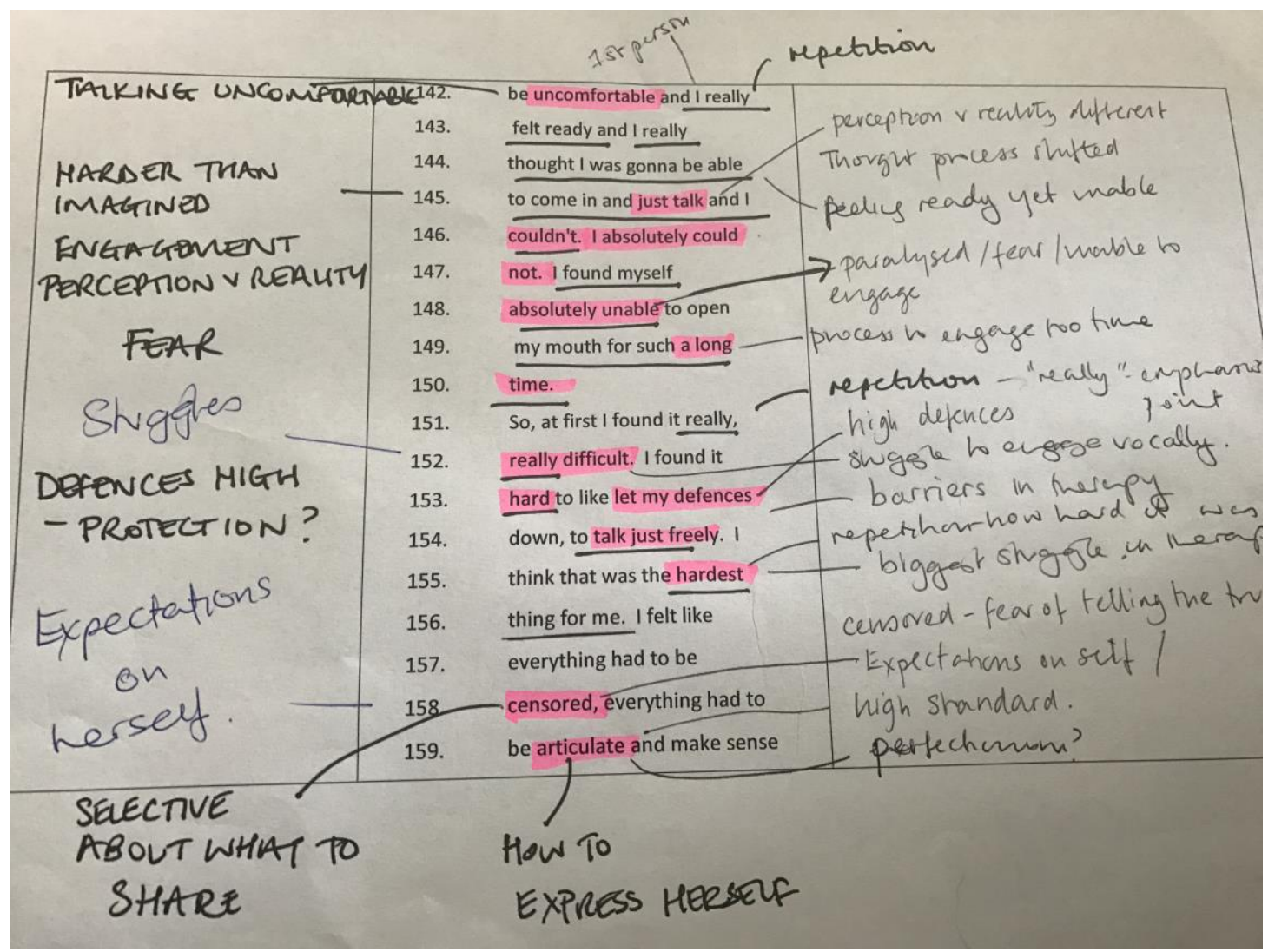

\subsubsection{Emergent themes}

Following the coding stage, the focus became the development of emerging themes. This involved organising and summarising the analysis, reducing the volumes of comments while retaining the complexity in the transcript and notes (Smith et al, 2009). I would list the themes and then move them around to form clusters of related themes. In the analysis, it was difficult to select what data to include, to ensure that the richness would be retained. The 'looser label' approach described by Larkin and Thompson (2012) meant that interpretation was done in a gradual manner, ensuring that deeper levels of analysis could occur as part of a larger hermeneutic cycle (Smith et al, 2009).

As part of the process, I made lists and lists on A4 and refined them gradually. Smith et al. (2009) detail how super-ordinate themes can be identified through 
Helpful and unhelpful processes in psychological therapy for female substance users

abstraction (putting like with like and developing a new name for the cluster); subsumption (where an emergent theme itself becomes a super-ordinate theme as it draws other related themes towards it); polarisation (examining transcripts for oppositional relationships); contextualisation (identifying the contextual or narrative elements within an analysis); numeration (the frequency with which a theme is supported) and function (themes are examined for their function).

\subsubsection{Cross Case Analysis}

Cross case analysis involved looking for patterns across each case. This was achieved by drawing up a list of themes and clustering these into master themes for each transcript. A table was created that listed the superordinate themes and quotes that reflected the meaning for each participant. I then started to compare the themes from each transcript to see if there were any correlations. As patterns began to emerge, I felt I had selected a small number of abstract, summarising themes that best captured my interpretation of the participants meaning. This final set of superordinate themes and subthemes that can be seen in Appendix 8.

This process of cross analysis was found to be challenging, as I wanted the titles to convey the most helpful and unhelpful aspects of psychological therapy from the voice of the participants. I felt that each interview had rich content and it was hard to differentiate between what to include and what to omit. I was also unsure about how abstract the titles were meant to be, therefore they did get changed a number of times. It was also time consuming as I returned to the transcripts repeatedly to ensure that I had not missed anything significant. With each stage, themes become a higher order, as emergent themes coalesce to form higher order themes. These themes begin to show connections and relationships for form superordinate themes. The final superordinate 
Helpful and unhelpful processes in psychological therapy for female substance users

themes selected were the ones I felt represented the phenomenological experiences of the women most accurately, and was achieved through IPA'S commitment to this approach.

By illustrating the analysis process step by step and by acknowledging the areas I found difficult, I am hoping to demonstrate how I have remained within an interpretive and phenomenological stance. By staying close to the women's accounts and by documenting all stages of the analytic process on paper, I ensured I was not imposing on the data or prioritising aspects of the participant's experience in order to fit my own view. Rather, I felt immersed in the process of engaging with the data aligning myself with the principles of IPA. Thus, my interpretations throughout the analysis are informed by my identity as a counselling psychologist, but the aim is for the reader to be able to distinguish between the women's experiences and my own.

\subsection{Personal context and Reflexivity}

As a counselling psychologist trainee, I have worked with female addicts and have seen first-hand the difficulties faced when trying to reach abstinence. My experience of having addiction in the family also had important repercussions for this research. It was important for me to consider my own preconceptions and biases when engaging with the women. I have witnessed the trauma and shame that underpins certain addictive behaviours, and the difficulty clients have in accessing their feelings without the safety blanket of drugs or alcohol. My reaction towards these women has always been one of compassion and a desire to know whether more could be achieved in supporting their needs within psychological therapy. 
Helpful and unhelpful processes in psychological therapy for female substance users

As I have worked with this client group, I was aware of my own attachment to women in addiction. Therefore, my capacity for reflexive thinking was a fundamental process in reducing the likelihood of imposing any of my beliefs onto the women's accounts (Willig, 2008). Throughout the interviews, I aimed to listen to the women and ensure that they felt safe. I was conscious of their vulnerability and wanted to provide the opportunity for their voices to be heard. As a trained addictions counsellor, I also had to consider my role as a researcher, keeping separate my natural instincts to act as a therapist in the room.

Reflexivity is the term used for explicit consideration of ways in which, it is likely the study, was influenced by the researcher (Smith, 2008). Reflexivity has been increasingly recognised as a crucial process in the generating knowledge by means of qualitative research (Ahmed Dunya et al., 2011; Blaxter et al., 2006; D’Cruz et al., 2007; Gerstl-Pepin and Patrizion, 2009; Hammersley and Atkinson, 2002; Horsburgh, 2003; Koch and Harrington, 1998). The project of examining how the researcher and intersubjective elements impinge on, and even transform research has been an important aspect in the growth of qualitative research (Finlay, 2002). Since it is acknowledged in qualitative research that the beliefs and assumptions of the researcher will influence how they collect and analyse data, it was important to be aware of my own beliefs and assumptions around addiction before I began the study. Hertz (1997) urges researchers to be aware of their own positions and interests and to explicitly situate themselves within the research. The following section therefore comprises a personal statement, written with these aims in mind. 
Helpful and unhelpful processes in psychological therapy for female substance users

\subsection{Self-reflexivity}

I am a thirty nine year old white British woman born in the north of England. I am heterosexual and have a young daughter and expecting a son. I am single parent and have not been married. Reflecting upon my own subjectivity in this research, I have thought at length why addiction is such a fascinating subject area to me, and my position in this research. Growing up in a northern town, drinking alcohol was part of the culture. My best friend came to live with me when he succumbed to alcoholism, at a time he had lost all other sources of support and wanted to end things. This experience gave me an insight into how addiction can easily take a hold of an individual, as well as reveal how difficult it is for someone to get access to help.

Two years ago, I separated from a long-term partner who was an alcoholic. His drinking lead to violence in the home, witnessed by both my daughter and I. Sadly, this had a huge impact on us both, and, perhaps as with all these relationships, I felt the drug had transformed him from a caring individual into an emotional and physical abuser. I have also recognised that I have wanted to fix my best friend and my ex-partner, but have been overcome with a sense of helplessness, reinforcing the belief that recovery can only be achieved when that individual wants to stop.

From a clinical perspective, my introduction to the field of mental health has led to me to work assisting homeless individuals with addiction, and to a placement within a women's only rehabilitation treatment centre for substance use disorder. The placement was followed by employment for two years, before starting my Doctorate in Counselling Psychology. Addiction has impacted my life on both a personal and professional level and I feel passionate about this client group. 
Helpful and unhelpful processes in psychological therapy for female substance users

From the onset of the study and after some research, I recognised that women had less support offered to them compared to men. Equally, they seemed less vocal about seeking psychological assistance, often due to fear of losing their children or the social stigma attached to addiction. As a woman and a therapist, I wondered how the women that had agreed to participate in my research, would feel about sharing their experience of psychological therapy and whether I would be asked personal questions before they began talking in the interview. I made a conscious decision not to disclose that I was trained as an addictions counsellor. I was worried that this knowledge could impact their answers, for example, by withholding information, or by looking for reassurance from me. I feared it could go from an interview to a counselling session if I let it. I was also concerned that my participants would ask if I was in recovery and my motivation towards conducting the study. I had genuine feelings and an interest in knowing how these women had been successful in treatment, especially given my own experiences, but I wondered if this was about my own fear of being distracted away from the research question and the interview becoming about me. Would the women pick up if I was 'one of them' or not? Would they trust me enough to disclose what they considered to be helpful and unhelpful aspects of psychological therapy?

When reflecting upon my own assumptions in relation to the research question, I wondered if the relationship between the participant and their therapist would emerge as the most helpful aspect of their psychological counselling. I also wondered if specific interventions within different therapeutic modalities would be influential to how they reached their successful outcome or whether they felt external factors outside of psychological therapy had the greater impact. I also questioned whether a participant's motivation to change would be influential in their outcome, as I have always believed that someone will stop using drugs or alcohol only when they are ready. Despite these 
Helpful and unhelpful processes in psychological therapy for female substance users

assumptions and how they could affect my bias, I was conscious of bracketing these thoughts and aspects of personal experience from working with this client group and to immerse myself fully in their accounts.

Knowing how difficult the addiction treatment process is and having come to my own conclusion that trauma underpins most addictive behaviour. I feel respect and admiration for women that have undergone treatment, as well as passionate about getting their voices heard. Despite the people in my life with this affliction being male, women face huge discrimination with this disorder. As soon as most of the women began talking about what had lead them into treatment, I wanted to say, "Well done, that's amazing", but had to monitor my feelings.

When discussing how therapy was helpful or unhelpful in this respect, it is hard not to feel empathic towards the participants. As part of the process of therapy, I have acknowledged the importance of research such as this. I know that the people in my life suffering with addiction may never seek support, but for those that do reach out, their psychological therapy could be vital in keeping them alive. It was also this viewpoint that allowed me to position the participants as central to this study. Despite my own experiences and role as the researcher, I had to remain focused on the participants' experience.

Upon reflecting on what I brought to the interviews, I felt that I was open and empathic managing to retain the focus on the helpful and unhelpful factors of psychological therapy. One participant did deviate from this, and talked in depth about what she had been through. I remember feeling anxious that I wasn't going to be able to bring the interview back to the topic. I was also surprised about how open many of the women were in their responses. The level of introspection and subjectivity reflected 
Helpful and unhelpful processes in psychological therapy for female substance users

an awareness these women had around their own psychological needs. I remember feeling so grateful that the women were engaging, and wondered if this has something to do with me being a woman who was interested in listening to their journey. I also found myself critical of my own experience as an addictions counsellor. As they shared what was helpful, I reflected on my own therapeutic interventions with clients and how differently I would have worked, had I had the knowledge I gained through conducting these interviews at that time 
Helpful and unhelpful processes in psychological therapy for female substance users

\section{CHAPTER FOUR:}

Findings

\subsection{Introduction}

The data analysis elicited a number of interrelated themes that demonstrated what the women found helpful and unhelpful in their psychological therapy for substance use disorder. Three master superordinate themes presented in this chapter include "What words can't express - finding another language", "Identification with my therapist" and "Getting towards acceptance".

The first theme "What words can't express - finding another language" encapsulates the difficulty the women had in finding their voice in psychological therapy and finding an alternative form of expression. "Identification with my therapist" was how the women perceived their relationship with the therapist and the impact that had on their motivation. "Getting towards acceptance," tells of the women's transition from resistance to acceptance during the psychological process. Those superordinate themes and the subordinate themes therein are presented in a table in Figure 2. Many themes emerged within individual transcripts and when the same themes appeared in at least half of the other transcripts, they were categorised as being recurrent. This was to illustrate an idiographic perspective, but also to counterbalance that perspective with more generic accounts.

In accordance with the IPA framework for analysis, an attempt has been made to represent each participant's voice whilst remaining sensitive to individual differences (Smith et al $2011 \mathrm{a}, \mathrm{b}$ ). In addition, these findings have aimed to capture the meaning of the phenomenon, (to the participant) whilst acknowledging my interpretation of the text as the researcher. 
Helpful and unhelpful processes in psychological therapy for female substance users

Table. 2. This table presents super-ordinate themes and corresponding subthemes. (Appendix 10)

Superordinate Theme 1: What words can't express; finding another language Subordinate themes: Overcoming the painful silence

I really thought I was going to be able to come in and just talk and I couldn't. I absolutely could not (Sam, 143)

\section{Helping to identify feelings}

When you finish talking in a painful conversation, there's a painful silence; when you do it with art there's a product infront of you that collates all that, the good and bad times.... (Emma, 685)

\section{Superordinate Theme 2: Identification with my therapist}

Subordinate themes: 'Been there, done that'

Because they had been there and done that, you know....they'd experienced what you'd experienced, be it with drink or drugs, they'd been in those proper depths of despair that you don't want to go back to (Louise, 551-553)

\section{Symbolising hope and professionalism}

This woman was, you know, together and lovely, and everything I didn't know an addict could have (Louise, 290)

Superordinate Theme 3: Getting towards acceptance

Subordinate themes: Resistance 
Helpful and unhelpful processes in psychological therapy for female substance users

I wasn't completely honest with my counsellor. She went by the notes I'd selectively chosen to share. They were horrific enough. Um...but what I didn't tell her was that actually I wanted to give my body a break and use successfully (Jules, 103)

\section{'she had me all along'}

My triggers are guys, you know. I get lost very quickly, controlled and I'm unheard. My counsellor asked me to think about my relationship with men but I just wanted to ignore her. Only now I'm clean do I understand how right she was. (Emma, 789).

Participants in this study were given pseudonyms. They are referred to as Sam, Anita, Emma, Kat, Jules, Louise, Rachel and Noreen.

\subsection{THEME 1: WHAT WORDS CAN'T EXPRESS; FINDING ANOTHER LANGUAGE}

This describes the difficulty the women found in expressing themselves in psychological therapy for substance use disorder. Overall, the participants reported an ongoing sense that they wanted to talk and open up around their feelings but were paralysed in sessions when it came to finding their voice. In order to communicate their needs, the women had to find alternative forms of expression to help them. The first subtheme describes the pain of their silence and their interpretation of why they found it so difficult to overcome. The second subtheme helping to identify feelings describes how the women worked with their counsellors to find methods that were helpful to them to access their narratives and how another language allowed them to work through their trauma towards recovery.

\subsubsection{OVERCOMING THE PAINFUL SILENCE}


Helpful and unhelpful processes in psychological therapy for female substance users

To overcome the silence in the therapeutic space was a stage that all the women mentioned. In the narratives, it was clear how difficult they perceived engaging verbally with their therapist when starting psychological therapy. When asked to pinpoint (Sam, 259) their feelings, their accounts described wanting the words to come out to their therapist, but feeling that a barrier (Louise, 98) existed. As they described this recollection, I was surprised at how difficult they had found it to describe their feelings. It felt that their defenses were either consciously or unconsciously preventing them from expressing themselves. However, the expectation each woman felt at the beginning was that they should have been able to talk and they expressed surprise they were unable to engage. For any clients engaging in therapy, the task of opening up can be daunting, however, this client group particularly spoke about the struggle (Anita, 77, Kat, 28, Noreen, 123) they faced when having to open up and express themselves, either due to the pressure they placed upon themselves to articulate (Noreen, 67, Sam, 159) their words in a particular way or an inability to even speak (Kat, 28, Rachel, 28) due to the pain (Anita, 428, Rachel, 90).

Over half of the women described how they had entered therapy and found themselves wanting to talk but described that part of them as shut down (Noreen 89, Rachel, 93). This gave a sense that they wanted to engage but that many could not convey what they had suppressed for years, despite their expectation upon themselves that it would be easier than it was.

I really thought I was going to be able to come in and just talk and I couldn't. I absolutely could not (Sam, 143) 
Helpful and unhelpful processes in psychological therapy for female substance users

In this quote, Sam alludes to the fact that being able to talk was a taken-forgranted aspect that most the women experienced when they arrived in therapy. The use of the word 'absolutely' stressing how much Sam wanted this conveyed. Only when engaged in psychological therapy did it become evident how challenging these women found it to share their voice and overcome the silence. Sam's narrative revealed her sense that words could not transmit her internal experience regardless of her desire to speak and her account focuses on a sense of frustration with her own inability to access her words, despite her best efforts to participate. Sam was not alone and many described themselves as numb (Jules, 360, Louise, 98, Emma 258), feeling distant from their emotions. In the past, drug use had been a way of escaping their feelings and that transition to being 'clean' from any substances meant that facing reality appeared to be a challenge.

Explicit in most accounts was concern around feeling supported in counselling and the impact this had on their desire to talk. It felt that their addiction had taken away their sense of knowing who they were and the ability to express their needs.

The kind of main factor why I kept using was because I was so frightened of actually who I was, so I never expressed what was inside, how would anyone understand? (Noreen, 28).

This demonstrates an essence of fear in the narratives, conveying both their vulnerability in accessing their suppressed feelings, combined with a belief that a therapist would not 'understand' and thus be able to support them. 
Helpful and unhelpful processes in psychological therapy for female substance users

You're not going to be able to hold it-easier to stay in the silence rather than open up and the risk of not being contained (Rachel, 394).

Rachel and Noreen's fear of expressing themselves and questioning whether their counsellor in psychological therapy could 'contain' their feelings reflected a dilemma expressed by a number of the women. In both narratives it felt their voices had never been heard and their coping strategy was to block any emotion, as the silence felt safer. Rachel's concern that her counsellor would not be able to "contain" her feelings was indicative of the risk that many felt within therapy. It appeared that Rachel had a real fear of knowing whether she would be heard and understood, and in common with the other women, there was anxiety about letting their guards down. If they opened up and accessed their feelings and did not feel supported, there was a high risk that they would go back to using drugs to 'numb' the pain (Jules, 68). It also felt that the women identified speaking as the only tool of communication, without being informed about alternative strategies to open up.

A number of the women's narratives revealed that their silence was underpinned by their past experiences of not being heard by previous therapists and their struggle to know what to communicate.

I've never allowed anyone in, in my whole childhood, so I really did struggle to sit with someone and open up my feelings (Anita, 49).

Anita's words gave an insight into how difficult it was engage and allow access of that part of her self to others. It was as if the women knew that they were expected to share their feelings and experiences of their drug using behaviour, but wanted to 
Helpful and unhelpful processes in psychological therapy for female substance users

avoid facing that reality, as it meant accessing the shame of my past (Kat, 901). Anita also had the belief that counselling was a way of dealing with the consequences (Anita, 72) of her past and this was seen as a struggle for these women to comprehend in reality. This idea that their addiction had taken them to the depths of despair (Louise, 247) and that psychological therapy was the only route to survival, generated a fear that some of the women struggled to face. In describing psychological therapy as dealing with the past, it appeared that it was helpful for these women to recognise the 'consequences' of where their addiction had taken them in order to heal themselves from the trauma, as it was a way of letting go of their past.

I was thinking away and keeping my mouth shut. (Anita, 386).

In Anita's case, her description of consciously sitting with her thoughts and refraining from expressing herself conveyed her difficulty in engaging verbally and her need to suppress her feelings. This idea of "keeping her mouth shut" felt like a defence mechanism in case she felt she would be judged "I did wonder if they thought I was beyond help" (Anita, 218). A fear of feeling vulnerable and wondering how her counsellor perceived her, left Anita feeling suicidal, that she had no hope. Anita described having experienced this with her previous counsellor and the impact had possibly been a contributing factor to an earlier relapse.

Everytime I open my mouth to tell them something I really needed them to hear, they'd kind of go to something completely different (Anita, 332). 
Helpful and unhelpful processes in psychological therapy for female substance users

This idea of feeling 'unheard' and 'misunderstood' (Anita, 336) by her previous therapist had a detrimental impact on her recovery as she had reverted back to her drug using behaviours and 'left me feeling ashamed' (Anita, 654). This insight into what Anita found unhelpful informed how important it was for these women to feel heard and feel valued in what they had to share. If they are denied that space or talked over, then these women are left feeling that what they really need to share is being ignored. A process that might reflect and replicate what they experienced in their past. The experience of being shut down by her previous counsellor had led to Anita sitting in silence with her new one. To overcome the silence, most of the women described working with their counsellors to find an alternative form of expression to access their emotional states.

When describing their experience, it was clear that the women perceived their addiction to be connected to an array of negative, intolerable emotions that underpinned their inability to find their voice. The women's accounts described how using drugs or alcohol had denied them access to the pain they felt and how the expectation to talk about their feelings left them vulnerable (Rachel, 291). This would mean that it was easier to sit in silence than be able to vocalise what they needed to say, especially if they felt their counsellor was not engaged. There was also a sense that being able to articulate what they needed to say was a difficulty. Hence it was not just being given the space, but also how to express their feelings was a struggle a number of the women faced. In the narratives, overcoming their painful silence was identified by all of the women as helpful for them in psychological therapy, and recognising that they needed to open up to progress. However, this idea of formulating their feelings into words was a huge task that many found overwhelming. When therapy is directive and unhelpful, 
Helpful and unhelpful processes in psychological therapy for female substance users

this is when the women are left in their silence and pain, and it is clear that this can have drastic consequences on their treatment outcome, as demonstrated by Anita.

\subsubsection{HELPING TO IDENTIFY FEELINGS}

This subordinate theme explores the difficulty the women felt in identifying their feelings within psychological therapy and how it connects with self-expression. It follows on from the first subtheme that encapsulated the women's fear of opening up to an unreliable container and focuses on the alternative interventions used that enabled those feelings to be accessed. Instead of verbally communicating their emotions, the women described alternative interventions as helpful in helping them progress forward.

In her account, Sam described the importance of having an alternative form of expression such as art and music, which she could use for interpretation and exploration. She also revealed how important this use of creative expression was for her to manage her recovery from SUD. In working with her counsellor, Sam describes how helpful this process was in psychological therapy as it allowed her to transform her feelings into something creative that protected her from relapsing and using drugs. For the women to be able to process their feelings in a safe way meant that they were able to handle their emotions in a way that kept them secure

Being able to identify how I feel, like the colours and stuff like that, makes such a big difference because when I am overwhelmed with an emotion, if I can't put my finger on it, that's the void that I can fill and want to fill with drugs (Sam, 397-400).

Sam's narrative revealed her need to identify her feelings through artistic expression. Similar to other women, her underlying message was her need to access 
Helpful and unhelpful processes in psychological therapy for female substance users

her feelings and have them explored in a way that did not leave her feeling too vulnerable or overwhelmed. Sam talks about 'filling a void' and this is a reminder that many of the women expressed a fear of relapse and linked their feelings to their drug using behaviour. The void Sam refers to, felt like a hole that drugs had left behind. Instead of using substances to numb their pain, they are left to process feelings but unable to articulate their needs into words. Sam talks about wanting to 'fill that void with drugs' and this represents an urge to use, which many of the women identified as a risk to their success. Finding another language such as creative art was paramount to Sam's personal transformation as it allowed her to work through issues that she could not have expressed otherwise.

These creative outlets for the women highlighted how having something tangible bypassed their conversational limits.

When you finish talking in a painful conversation, there's a painful silence; when you do it with art there's a product infront of you that collates all that, the good and bad times.... (Emma, 698)

Emma identified how helpful it was for her to collate all her feelings into a product that she could take into psychological therapy. Overcoming the barrier she faced with words, her artistic expression had allowed her to face the 'good' and 'bad' aspects of her addiction. In recounting this experience, Emma identified how helpful it was for her to have an object to identify with. When talking about the 'good,' it felt that she was referring to being abstinent and her inner strength. The 'bad' represented the pain and shame her addiction had taken her to. By working on her art, Emma 
Helpful and unhelpful processes in psychological therapy for female substance users

managed to overcome her 'painful silence' and the feelings she was avoiding could be addressed. It is possible that this was a way of attributing meaning to an artistic object that she had made, represented her difficulties and helped with her healing process.

It appeared that the sense of bringing something external into therapy was seen as helpful for these women in terms of communication. It was a way of bringing the external in when words were not enough. When exploring why this is helpful to this client group, I felt it linked with the intolerable feelings that they carried. In having their feelings represented in a product, it meant taking the focus away from the women themselves. Instead of having to verbalise what they felt, this product was an expression of themselves without having to explicitly describe it. Having an external object also meant they had a focus, replacing an unhelpful silence that had existed previously in sessions. This focus on something created by them, encompassed the part of themselves that they were unable to reach verbally. Through externalisation, it enabled their feelings to be processed from a different perspective.

This recognition of a 'song' or piece of 'art' was something nearly all the women described as helpful in their sessions, yet it was about something far deeper than just a creative outlet. The association with an object to their feelings allowed the women to deal with some of their suppressed pain and trauma that they previously would have used drugs or alcohol to avoid. This was significant as these women could only make the link between their despair by having a product to encapsulate their emotions. It gave a sense of how difficult it was for this client group to access their feelings and how helpful they found this alternative form of expression in their journey towards recovery. 
Helpful and unhelpful processes in psychological therapy for female substance users

I could draw scenes or scenarios. I've erm, spoke about colours, anything like that with association...because when my feelings came back, my head would not. I thought I was going insane (Noreen, 248-252).

This quote felt important, as Noreen highlighted how her feelings could not always be processed within her mind. The word 'insane' reflected her fear she was losing her mind and that her therapist would not be able to hold her within her session. It also gave a sense of the disconnection felt between the mind and the feelings and the difficulty in collaborating both within a session. For Noreen, art was a useful tool to associate colours with how she felt, despite her inability to make sense of it cognitively.

For some women, their feelings could be expressed by identifying with another persons creative work. Jules, for example said: to chose a song that relates to a subject and play it infront of others can be scary and can show a lot of you and say a lot more than you ever could (Jules, 289). This quote suggests that Jules discovered a lot more about herself, that she would not have accessed through just words. When asked what did you find helpful about psychological therapy, she talked about how therapeutic she found relating a song to a subject. The process of sharing with her counsellor may have felt scary however she identified how it could express more about her internal world than she had the ability to do through speech. Similar to Jules, most of the women described music or art as something massive (Jules, 367, Sam, 417, Katrina, 302). The word massive was often repeated in their narratives giving me a sense of how important and helpful it was in their recovery. It felt that they wanted me to know how significant other forms of expression were. In overcoming the emotional difficulties they faced in 
Helpful and unhelpful processes in psychological therapy for female substance users

the past, especially the shame that underpinned most their accounts, meant feelings could not be expressed through words, however a song or art piece seemed to expose them and encapsulate how they felt without jeopardising their safety or desire to use.

\subsection{THEME TWO - IDENTIFICATION WITH MY COUNSELLOR}

This second theme identification with my counsellor featured strongly across most participant accounts. It reflected the importance of the women's relationship with the counsellor, in terms of identification and the helpful impact it had on their psychological therapy. The sub-theme 'been there, done that' was a quote taken from Rachel that encompassed how many women felt when they discovered their counsellor was also in recovery. The second subtheme symbolising hope and professionalism was how their identification with their counsellor was helpful as it represented hope and a life beyond which they had known.

\subsection{1 'BEEN THERE, DONE THAT'}

Having a counsellor who identified or appeared to be in 'recovery' was significant to whether many of the women perceived their psychological therapy as being helpful or not. As the women talked about discovering this information about their counsellor, their voices became animated as if it represented a real turning point in their therapy. It was as if they could not believe that their therapist could have shared the same experience. It was clear from all the accounts that this connection led to a shift in the dynamics, as it was not just a therapist and client who sat together, but two female addicts, who might embody hope that they too might recover. Further exploration revealed how massive (Jules, 292) it was for them to know if their counsellor was in recovery (Emma, 199) or had some experience (Sam, 983) of addiction in some way. 
Helpful and unhelpful processes in psychological therapy for female substance users

When this was explored further to identify why it was helpful, there was a sense that knowing their therapist had been there, done that (Rachel, 630) meant they felt understood and in safer hands.

Initially, many of the women described not knowing whether their counsellor was in recovery from substance use disorder. It was only later during therapy when the counsellors either disclosed, or the women had a sense that they were similar, that meant a shift in the relational dynamic occurred, and they did not feel so alone.

It did help the fact that I knew she was in recovery; that really made a difference. I actually found out halfway through treatment and when I did I thought..you know, she knows (Emma, 214-222).

Emma talks about this idea of 'she knows' as something that came as a revelation. She describes that the discovery was helpful for her as it made a difference to how she perceived her counsellor. Having that partial identification allowed Emma to feel understood. This recognition that they shared the same disorder was something she described as bringing her closer to her counsellor as she knew she understood.

She's just a beautiful, graceful, intelligent woman, whose stuck needles in places where I have, and been to the depths I have (Emma, 1312)

For these women, they perceived their addiction as destructive and shameful and having someone who could really understand what they had been through and had shared the depths that addiction had taken them to, made that process easier. From the narratives, this knowledge allowed them to connect without having to explain or 
Helpful and unhelpful processes in psychological therapy for female substance users

describe in detail parts that a non-addict counsellor may not have understood. This person that they had in front of them had 'been there and done that' and they were not alone.

It's like they understand. It's not so shameful. It's like they are telling me it is ok; it's alright. (Anita, 907).

From Anita's quote, knowing someone she has a positive relationship with has had an addiction makes her feel that she herself must be worth more, and feel shame less, through the empathetic connection. This was a powerful factor in helping the women to be able to disclose their past and motivating them to engage more in the therapy. There was also a sense from the women that this 'understanding,' meant their therapist would have a greater idea around what they needed in treatment to succeed. The analysis revealed that a number of the women felt that it was a sign that they were going to recover. Instead of feeling judged or having someone who had no experience of using substances, the women found it helpful that there was this identification. It created a bond and acceptance that they were not alone. Having someone who appeared to share the same implicit beliefs and goals meant a mutual respect in the relationship because we were the same (Emma, 237). It was also felt that someone who had studied to be a therapist and had not had that experience would not have the same understanding, as they could not understand the complexity of addiction and its pitfalls.

She just had this way of looking at you and going 'I know what your thinking and feeling, don't try and deny nothing with me' (Rachel, 1320). 
Helpful and unhelpful processes in psychological therapy for female substance users

Rachel's perception that her counsellor knew how she felt internally without her explicit expression is what she identified as helpful in therapy. She could not hide behind her fears, because the counsellor would be able to see through her. This was important as it highlights a sense of understanding that exists between the therapist and the client that was not explicitly acknowledged by anything more than a look. When overcoming the shame and finding her voice in psychological therapy, Rachel described finding it helpful that her therapist was on the same level, as it meant that she knew she had to be honest. Knowing that her therapist had overcome her addiction and was also working at staying sober meant there was an affiliation and that there was no place to conceal her true feelings.

The importance of a relationship with a therapist that has 'been there, done that' was illustrated when two women disclosed that they told their first counsellors bullshit (Sam, 88, Noreen, 96) when they began therapy. This was either done by not disclosing the truth or being selective around what content they shared. Interestingly, when Sam and Noreen both changed counsellors and began working with women they knew were in recovery, it was only then they admitted they 'couldn't get away with their behaviour' anymore. This again illustrated the sentiment that the women felt a greater chance of succeeding if they knew their counsellor had the experience and tools for dealing with the unknown and how unhelpful it was if they felt no identification. Working with a therapist that had 'been there, done that' meant they could not get away with denial or avoidant behaviour, aspects that other counsellors without the shared same experience, had not been able to identify. 
Helpful and unhelpful processes in psychological therapy for female substance users

I know there are good counsellors, good therapists that are not in recovery. I think once, when I know, I kind of feel more safe because I know that they, they've been through it (Jules 328)

This quote highlights the impact of knowing someone has 'been there, done that' in terms of feel safe. As this client group present as vulnerable and fragile, safety can be paramount. Many of the women had been through traumatic relationships, prison or sex working and described never feeling safe. Jule's narrative revealed that for her, knowing her counsellor had been through it helped, as she felt her therapist had a greater understanding of her behaviours and her triggers to relapse. In feeling safe Jules had the confidence to be open and honest which she ascribed to having that connection. There was a sense that a therapist with a shared experience could identify risks and potential triggers and utilise this knowledge in therapy to keep her safe.

Because they had been there and done that, you know....they'd experienced what you'd experienced, be it with drink or drugs, they'd been in those proper depths of despair that you don't want to go back to (Louise, 551-553)

Louise talked about how helpful it was for her to have this identification with her therapist as it meant she could relate and not feel embarrassed about what she shared. Similar to Rachel, she described how working with a counsellor who had been there was important in understanding her proper depths of despair. There was a sense from her words that she had experienced such trauma through her addiction that she needed someone to identify with that dark place without feeling judged. Throughout the extracts, this belief or perception that their counsellor was the 'same' was identified 
Helpful and unhelpful processes in psychological therapy for female substance users

as helpful in the therapy. This similarity led to a shift in the relational and was recognised as a contributing factor towards change in the psychological process.

\subsubsection{SYMBOLISING HOPE AND PROFESSIONALISM}

This woman was, you know, together and lovely, and everything I didn't know an addict could have (Louise, 290)

This subtheme gives an indication of how much the women valued their identification with their counsellor and how it helpful it was, in terms of symbolising hope and professionalism. It extends this idea of someone who can see through their façade, as the shared connection resulted in a more authentic presentation. In this quotation, Louise alludes to the way in which the perception of an "addict" is regarded as the opposite of lovely and together and her surprise that her therapist could identify as both a professional, an addict and represent something positive. There was a sense from the narratives that she was not alone in her belief that an addict signified someone crazy (Kat, 543) different (Rachel, 193) or shameful (Anita, 277), all negative connotations. Therefore, to have a therapist that disputed that belief and had survived was hugely helpful as it instilled hope that in their future they also could be that person. The ability of the therapist to demonstrate fallibility and balance allowed the women to feel that they could do the same. There was a sense that the women needed the therapist to be the same yet different. Due to the boundaries in the therapeutic space, the women could identify with their therapist as someone they could aspire to, yet the difference also allowed them to have an interpersonal relationship that enabled them to change. 
Helpful and unhelpful processes in psychological therapy for female substance users

Not everyone's experience is the same, but she made me see there is another side, there is hope (Kat, 341)

This quote from Kat demonstrated how she found it helpful that her counsellor represented another face of addiction. Even though the women recognised hope as an implicit part of the therapeutic process, many of the accounts gave a sense of isolation linked to the despair that their using made them feel. The words 'not everyone's experience is the same' conveyed Kat's understanding that her experience was personal, yet her therapist represented something that she had previously felt out of her reach - a world of hope, recovery and employment. This felt significant as many of the accounts demonstrated the struggle to believe the women could have an identity other than as a drug user.

She was someone I could learn from (Jules, 746)

As Jules talked about her counsellor, it felt that she symbolised something more than just a person. The description that she was someone that I could learn from indicated that Jules had someone to aspire to be like. Listening to the women it felt that having a positive role model in their world of addiction and negativity was an inspiration for their own recovery. It instilled the belief that they could have an identity other than a drug user and it felt that this was helpful to them when finding their own motivation to change.

Along with this form of identification with their therapist, the women's need to see their counsellors as 'professional' (Sam, 939, Kat, 565) and not as a peer was also evident in the narratives. This idea of professional alluded to the counsellor being qualified and with knowledge of addiction. 
Helpful and unhelpful processes in psychological therapy for female substance users

They are trained, which means that you know, there's confidentiality (Anita, 304)

As well as feeling safer and more understood, Anita's quote highlights her underlying vulnerability. The idea that nothing could be discussed beyond the therapeutic boundaries was helpful to her as it meant she felt contained. Her account revealed her struggle to trust and her desire for confidentiality. Throughout the extracts this was important, as the women expressed their need to know that they could share their trauma and feel safe. Anita's belief conveyed her need for her therapist to be trained and to know that whatever she shared would not go further. Many of the women described feeling disconnected with themselves as they described their addiction as all consuming and dysfunctional, so to have someone who understood the addiction, and the 'language,' who was trained, was helpful in how they could relate and view themselves.

If I felt I was talking to someone on a personal level, my fears around being judged would really come into play and would potentially stop me from talking about what I needed to talk about (Sam, 1003-1010)

Sam's narrative revealed how she needed to trust in order to open up. Even though there was identification with her counsellor, there was a clear distinction between having someone professional and someone as a friend. This seemed to evoke fears of being judged if someone was relating on a personal level. Sam also talked about how she would use different content if talking to her friends, which was also a theme with the other women. She identified how she would be more casual with friends and would not have allowed herself to get vulnerable. In contrast, if she felt safe with a counsellor, then she talked about allowing her vulnerability to emerge. Sam's account highlighted the link between being judged and feeling shut down. Having boundaries 
Helpful and unhelpful processes in psychological therapy for female substance users

in the relationship and an expectation of roles was seen as helpful in opening up their wounds.

I didn't need a friend as much as a counsellor and to feel like I was in therapy (Emma, 75)

From her narrative, Emma disclosed how helpful it was to feel that she was actually in a therapeutic relationship with someone trained. Despite feeling that she identified with her counsellor in terms of their addiction, it seemed there had to be some differentiation to feel safe. This supported the other women's accounts that the dynamic of having someone that acted like a friend would not work for their recovery. Emma described her need for boundaries and containment and feared that having a 'friend' would heighten her vulnerability. Having that distinction between a 'friend' and 'counsellor' was paramount to change, as a friend meant someone they couldn't get angry or feel guilty towards, feelings that could be evoked and worked through safely only with a trained therapist.

Linked with the aforementioned, several women described what they found as unhelpful when identifying with their counsellor. Despite the hope they symbolised for most of the women, some of the therapists were criticised for being too sympathetic (Sam, 527, Katrina, 798) or acting as a mother figure (Jules, 116), both factors inhibiting their authenticity in the therapy room. For Jules, it tapped into her relationship with her own mother and wanting to please her. This left her disclosing that she struggled to confront or share information that she felt ashamed of. Jules also talked about her fear of attachment and her ending in therapy, as it mirrored letting go of her own mother. Even though there was identification with her counsellor, having someone that re-enacted this role was detrimental to her recovery. Significantly, in 
Helpful and unhelpful processes in psychological therapy for female substance users

Jules's case, her counsellor had to leave during her therapy and she was allocated another. She talked about the trauma of letting go, but recognised that she had found that experience helpful as she had processed through her own issues of being a mother, her sense of abandonment from her own and the transference that had manifested itself with her first therapist.

Sam and Katrina both said that "sympathy" was unhelpful; they defined sympathy as evoking feelings of being seen as a victim and this was unhelpful therapeutically. There was a sense that this left the women feeling disempowered as it allowed their counsellor to represent a position of power. Instead of feeling equal in the room, the women described how unhelpful it was for their counsellor to make a judgement about how they felt and treat them with pity. Instead of enabling them to gain the tools to grow, they were left feeling their addiction was being seen as a moral failing. In both narratives, they alluded to taking time to work through this emotion only allowing their irritation to diminish as their psychological relationships strengthened.

\subsection{THEME THREE: GETTING TOWARDS ACCEPTANCE:}

This theme captures the transition in the women's attitudes from resistance to acceptance of both themselves and psychological therapy, and how this was helpful retrospectively for the women. Their narratives conveyed their rejection of the 'addict' identity, a label that represented acceptance of their medical diagnosis and a stigma in society. This theme is about how they entered therapy with different expectations of the outcome and the process they undertook to enable that change. 
Helpful and unhelpful processes in psychological therapy for female substance users

This links to the other subthemes as it highlights the challenges in psychological therapy and the role of the self in their transition to change. It illustrates the attempt to resolve these difficulties and how these women acknowledged that their own attitudes were not helpful in the process. It also demonstrates how much is connected to perceptions, their perception of self in the counselling dynamic and how their own attitudes were instrumental in their change.

\subsubsection{RESISTANCE}

This subtheme was only identified when the women were looking back at their attitude when they first started to engage in psychological therapy and how their defences impacted their engagement. As the women talked about their resistance it felt there was something deeper around confronting their fears. I sensed that a number of the women needed to test or push their counsellors to see if they could be held emotionally, before they would open up. Interpreting this behaviour, it felt that the women were scared of letting down their defences, as these are what they had used for survival.

I wasn't completely honest with my counsellor. She went by the notes I'd selectively chosen to share. They were horrific enough. Um...but what I didn't tell her was that actually I wanted to give my body a break and use successfully (Jules, 103)

In this quote, Jules reveals how her attitude was ambivalent at the start of her therapy as she talked about keeping aspects of her self concealed. She made a conscious decision to only share the parts of herself that she had selected, gave an insight into the wounds she talked about needing to hide. Her description of her notes as horrific 
Helpful and unhelpful processes in psychological therapy for female substance users

enough alluded to the trauma she experienced and the difficulty in sharing it as a whole. That the trauma was selected illuminated a sense of her suffering.

It felt from her words that Jules did not have the self-belief that she could succeed and her intention to have a break then use successfully indicating a paradox. Her words convey the horrific damage that her addiction had already done to her, yet she began psychological therapy with the illusion she could use successfully upon completion. When exploring this further, it felt that this aspect of her self was a defence against feeling exposed, a feeling replicated by many of the women.

Connected with these feelings was her perception that she was in control of the therapy. Jules conveyed that she had selected the notes, had shared the aspect of herself that she had wanted and intended to use again upon completion. Her resistance highlighted a need to feel safe by enforcing her own boundaries in therapy. Even as she talked, it felt that she had not recognised the barrier she had created until she was in a place of sobriety and could reflect on her journey retrospectively.

From the outset, most of the women expressed their struggle to engage in therapy because they either saw it as 'just another attempt at recovery' (Jules, 67, Rache;, 69) or a way to 'give my body a break' (Jules, 103) from using substances. This lack of hope in their own ability to succeed manifested itself in a resistance to participate. For others, it was their 'rock bottom' (Louise, 267) (Anita, 543), a term that symbolised their lowest point, often a situation of life or death. Even though these women needed the help, their resistance was rooted far deeper psychologically as it was representing overcoming the defenses they had in place that had quite possibly kept them alive. 
Helpful and unhelpful processes in psychological therapy for female substance users

For individual reasons, some women detailed how they had shown some resistance but had not explicitly realised this until they reflected upon their psychological therapy.

I didn't fully appreciate I was an addict of any sort (Emma, 17).

The denial of accepting her addict persona had left Emma questioning whether she could identify as someone different, and this impacted her initial engagement in her therapeutic sessions. As someone that had been using drugs for twenty-eight years, it felt from her words that she was unable to visualise any other reality. This only shifted as the relationship with her counsellor strengthened.

At some point in therapy their resistance began to be replaced by acceptance when they realised they were on the right path and their counsellor had understood them 'all along'. When describing this revelation, many of the women acknowledged that something far deeper transpired in their journey. The challenges they had faced and the reasons they resisted were helpful in getting to that point where they could now embrace their life without using substances.

At first I didn't want to be here because I had no boundaries and I had no structure....in the beginning I was a bit, oh god...I'm just going to be one of the addicts out there... (Kat, 651)

Kat's quote supports this idea that there was a sense of hopelessness at the start of psychological therapy that eluded her from completely engaging. This is important, as this unhelpful attitude may keep many women sustaining their addiction, as they do not believe they can succeed. This idea that she knew what to expect in psychological therapy and the belief that it would not work kept her defences in play. Kat's belief in 
Helpful and unhelpful processes in psychological therapy for female substance users

no structure or boundaries could be interpreted as her way of avoiding bringing herself into therapy. Her resistance was underpinned by her fear that she did not have the qualities needed to succeed. However, Kat does talk about this being her attitude in the beginning and that she recognised how unhelpful this was retrospectively.

I couldn't understand why I had to talk about it. I thought I just needed to block it out and they'd go off. I didn't realise how important it was to free yourself from that pain (Rachel, 428)

This quotation demonstrates the way in which Rachel's resistance manifested itself in her desire not to talk. This differentiates from being able to engage but finding herself unable to communicate, as in the first theme. Instead of being able to communicate, Rachel used avoidance to negate her painful feelings in the hope they would vanish. In common with many of the women, her resistance felt like a defence mechanism that had enabled her to survive through her years of addiction. Recounting her difficulty, Rachel talked about not being able to understand why she had to talk about her issues, which indicates how challenging she found psychological therapy. Her narrative reveals a fear of shame and vulnerability associated with her pain.

In line with the other quotations, this idea of resistance feels like subconscious behaviour that underpins the women's fragility. It suggests that their unhelpful attitudes were more about masking their fear of being able to deal with psychological therapy and the reality of being sober, as the drugs would have provided a security against their feelings. When Rachel talks about how important she found it to free herself from her pain, it illustrates how these women almost sabotaged their sessions because they were unable to see the benefits, only recognising the gains at a much later stage. At a deeper level, the trauma that underpins most the women's accounts seems 
Helpful and unhelpful processes in psychological therapy for female substance users

to connect with their resistance at the beginning. It did not feel as though the women denied the existence of the problem, rather, it was a lack of self-efficacy that they could achieve their goal of treatment.

The way Rachel talks about her realisation that psychological therapy was helping her and allowing her to process her pain illustrates her acceptance that things could be different, and could signify her attempt to get in touch with the healing part of herself.

\subsection{2 'SHE HAD ME ALL ALONG'}

This subtheme focuses on the idea that their therapist had understood their needs even when they themselves had not realised or wanted to recognise what was helpful to their recovery within psychological therapy. The transition towards acceptance of their identity as an addict meant the women often challenged the process by 'bullshitting' (Sam, 88) their counsellor or 'manipulating' (Louise, 288) their way through sessions. This theme explores how the therapist got them to the point of acceptance and how the women only recognised that their therapist knew what they were talking about at a much later stage.

I think I knew she was right and I didn't want her to be. I just really didn't you know. I wanted to....to...go off into this la la land and everything be ok (Noreen, 423-431)

This quote from Noreen illustrates the difficulty she felt within the relational. When describing how she wanted to go into la la land, Noreen is conveying her difficulty in being able to engage in psychological therapy and her desire to escape and become fixed without needing to play a part. It highlights how she came into therapy wanting something to be different, but did not want to actively do something about it. 
Helpful and unhelpful processes in psychological therapy for female substance users

This could be connected with the defences in place that were discussed in the first theme. Despite wanting everything be ok Noreen describes not wanting her therapist to be right, I really didn't. The fact that Noreen wanted her counsellor not to be right felt like ambivalence on Noreen's part to her own recovery. It felt that she was projecting her concerns around her own fear of failure onto the therapist as a security blanket. If her therapist failed her, then it would alleviate any blame if she relapsed. This seemed to be a common theme from the women, as it meant that they could avoid taking responsibility for the therapy if anything went wrong. It also highlights this dichotomy between wanting recovery and not wanting to engage.

When asked how this was helpful, Noreen talked about her realisation that her counsellor was right and how that led to a shift in her reality. Instead of having lala land as something that would save her, Noreen talked about grudgingly accepting that her counsellor did understand and discussed how this seemed to amplify feelings of letting go and succumbing to the process. In acknowledging that her counsellor did know best, it did not feel that Noreen was implying there was a power dynamic, rather, Noreen's acceptance that her recovery was dependent on a therapist who knew what she needed to keep herself safe. Even though Noreen talks about resisting this help, she retrospectively talked about how her attitude was alluding to something deeper within her that she was struggling to face.

She had this great knack of noticing when I was trying to brush over a subject, if I tried to use humour when painful, which I did you know. (Louise, 440-445)

Following on from this idea that the therapist was right, Louise recounts how helpful she found it having a therapist who could identify her avoidance in psychological therapy. Louise describes how she used humour to deflect her painful 
Helpful and unhelpful processes in psychological therapy for female substance users

feelings and how her therapist could see through her behaviour. This sense that her therapist understood meant exploring aspects of herself that she struggled to engage with. These could have been the parts that had maintained her addiction. Louise's acknowledgment that she did try to deflect her pain with humour highlighted her awareness that she needed a therapist who could read between the lines and understand what she was avoiding. Similarly, Sam describes how in retrospect she came to understand her need for boundaries and stuff like that as she would bullshit in a session to avoid her pain

Once I was with someone who was a bit stricter, who had kind of more boundaries and stuff like that.... I couldn't get away with that. It's like when I was doing that, I really felt she could see I was doing that (Sam, 93).

This quote highlights how Sam pushed boundaries with her therapist but realised that she could not succeed as she felt she could see what I was doing. Again, this was an avoidance tactic used to evade her painful feelings. In having a counsellor who was stricter, she describes how the dynamic shifted forcing her to accept she could not get away with that when working towards her recovery.

My triggers are guys, you know. I get lost very quickly, controlled and I'm unheard. My counsellor asked me to think about my relationship with men but I just wanted to ignore her. Only now I'm clean do I understand how right she was. (Emma, 789).

This idea of being asked to explore aspects of themselves that they didn't want to face is illustrated in Emma's quote. As she talked about looking back at her struggle around men, it felt that she was describing her two identities. The addict part that wanted to continue with certain behaviours, and the Emma in recovery that was only 
Helpful and unhelpful processes in psychological therapy for female substance users

able to see the risks upon completion. In identifying men as one of her triggers, Emma talked about wanting to ignore that part that needed to change. Similar to the other women's accounts, there is an aspect that when they begin psychological therapy they struggle to accept the process and to let go of the security of certain behaviours. The dichotomy of wanting someone to guide them in therapy and losing themselves in the process is a theme throughout the accounts. Despite this, a shift began when they found the balance between dependency and taking some form of control. This is when the resistance eased and acceptance emerged.

Emma talked about being lost, controlled and unheard and these are all words that could describe her life of addiction. The hold that drugs had placed upon her mirrored her role within her relationships and her lost self within the therapy room. From her words, she only understood and accepted how right her counsellor had been once she was clean. Her acceptance that her counsellor knew her needs seemed to elude not just Emma, but a number of the women until they had embraced their recovery and could identify these aspects for themselves. The ambivalence was replaced by acceptance only when they recognised that the counsellor had identified their needs a long time before they themselves had identified them. The shift in Emma's description from ignoring her counsellor to understanding how right she was gave the sense that Emma needed that time to really understand her recovery. Having a counsellor who had been right all along felt like it gave the women hope and the tools to maintain their recovery and the acceptance that their therapist had understood their needs and guided them on the right path. 
Helpful and unhelpful processes in psychological therapy for female substance users

\section{CHAPTER FIVE:}

\section{DISCUSSION}

\subsection{Chapter Overview}

In this final chapter, the analysis is discussed in relation to the existing literature. A critique of the methodology and process of the study is presented and the researcher's final reflections are incorporated. Furthermore, implications for clinical practice and suggestions for future research are given and the chapter ends with final reflections and a concluding section to the thesis.

\subsection{Situating Findings within the Literature}

In the introductory chapter of this thesis, the various debates about the concept of addiction and treatment models were discussed. This final chapter discusses the findings in relation to the research question, "What do women diagnosed with SUD find helpful and unhelpful in psychological therapy?" and aims to summarise the contribution I have made to the literature.

This section presents how the women's subjectivity was experienced and negotiated, progressing from a detailed exploration of the analysis to the implications for clinical practice. The women provided rich accounts of the aspects they found helpful and unhelpful in psychological therapy, producing data in a previously unexplored area. It should be noted that this thesis does not make claim of empirical generalisability. Instead these findings provide a platform for my interpretation of the analysis within the context of SUD literature and how these findings can be applied to clinical practice. 
Helpful and unhelpful processes in psychological therapy for female substance users

Throughout the analysis, I noticed the various ways in which the meaning of psychological therapy for the women was entwined with negative affect and emotional dysregulation, two core features of addiction (Thomas et al, 2016). This adds to existing research that suggests that these variables are barriers to SUD treatment success (Paulus et al, 2016). In the discourses, the interpersonal relationship with their therapist and the women's emotional state, were both found to impact their level of engagement within therapy. This supports the research that the working alliance is one of the most important factors in retaining SUD clients in treatment, as it establishes trust and security for the clients to open up (De Weert-Van Oene et al., 2001, NICE, 2015). Having a therapist who could work through their defences and normalise the shame, meant that this interaction enabled the women to understand their behaviours and what aspects they needed to change. Working with a therapist who they perceived to also be in 'recovery' was instrumental in feeling supported.

For many of the female participants, it was easier to identify the aspects of psychological therapy they found helpful rather than unhelpful. This could have been attributed to the positive place they currently found themselves in. Radley and Taylor (2003) suggest that the act of remembering and reconstructing one's biography in the process of recovery is not only a remaking of the past, but also fulfils the needs of the present. I noted that many of the women used the interview to express their gratitude towards their therapists and that recalling the experience of therapy reinforced how far they had come.

The first theme: 'what words can't express; finding another language,' emerged as all the participants detailed their difficulty expressing their feelings verbally. Interestingly, each participant attributed this difficulty to different reasons, such as 
Helpful and unhelpful processes in psychological therapy for female substance users

negative past experience, an inability to articulate their words, or simply finding it too painful to engage. This was an issue they all experienced at the earliest stage of psychological therapy. The importance of retaining a client in the first two sessions is illustrated by statistics that show between 20 and $57 \%$ of therapy clients leave after the first session and another 37 to $45 \%$ attend only two in total (Schwartz et al., 2001). For SUD clients, $50 \%$ fail to return after the second session (Miller \& Rollnick, 2002; White, 2005). Therefore, for this client group, overcoming the painful silence was imperative to remaining in therapy and retaining engagement. The analysis revealed that a number of the women were resistant and were unable to express themselves early on in therapy. Therefore, the clinical implication at the beginning of the work is to place a focus on keeping the client engaged.

Interpretation of their narrative in this first theme seemed to suggest that the women were demonstrating some level of resistance, either consciously or unconsciously, towards the therapeutic process and being able to open up to their therapist. Freud describes this resistance as an unconscious defence mechanism that surfaces when clients face changes that make them feel uncomfortable (Freud, 1959). On in-depth examination of the interview data, it was apparent how difficult the women found the expectation and reality of talking, and how easily silence could prevail. What helped these women was their therapist's use of interventions that enabled them to open up. There was also a sense that they felt relief that their therapist didn't give up and interpret their silence as lack of interest.

As the interviews progressed, the resistance they felt and overcame became clearer throughout all the narratives. As the women talked about their struggle to identify painful feelings, I wondered if initially this struggle reflected a degree of 
Helpful and unhelpful processes in psychological therapy for female substance users

internal conflict regarding change, as supported by research with those diagnosed with SUD (Miller \& Rollnick, 2002). However, most of the women talked about psychological therapy as something positive, even described as 'life saving', and only one woman regarded it as something that was a 'stop gap' from using. Her opinion shifted upon completion of therapy. Even though the women knew that this process was helpful, there was still an initial disparity between recognising their need to be there and their struggle to enagage, hence the importance of therapist interventions designed around the aims of engagement.

Research on the reasons why SUD clients struggle in therapy range from mandated status; lack of motivation to discontinue their drug use, discomfort opening up to strangers; co-occurring conditions, multiple diagnoses, initial therapeutic approaches that have increased resistance and confrontation techniques that left them feeling defensive (O’Connell \& Beyer, 2002). Interpreting the results of the current research, it felt that a number of these variables applied. However, the findings went beyond this previous study and explored what aspects of psychological therapy helped women to overcome these difficulties, such as alternative forms of expression and ways of overcoming their resistance. As well as recognising aspects of the process that enabled them to strengthen their recovery, all the women recognised that negative feelings and trauma were the root of their drug use, and therefore working through their painful emotions was seen as the most helpful aspect of their counselling.

While the first sub theme focused upon the participant's fear of opening up, the second explored how these women overcame this process. Alternative forms of expression were identified by all the participants as a way of managing their negative feelings and used as a tool for interpretation and exploration. For other women, it was 
Helpful and unhelpful processes in psychological therapy for female substance users

having a song, picture or a product that represented the 'good and bad' that could be used as a means to transform their internalised feelings to the external. This was identified as a helpful aspect in psychological therapy, as the women felt it gave their respective therapist a tool to see beyond their symptoms.

Art therapy was recognised by the women as helpful for contemplation and enabled them to correlate their feelings into a product, within the therapeutic process. A drawing was able to express what words were unable to say. Having an object meant they had something tangible to communicate their pain. The implementation of evidence-based practices in the treatment of SUD has meant alternative practices have been given little consideration (Alterais et al., 2014). However, the use of creative modalities have been found to assist SUD clients in their therapeutic process (Canning, 1999) by decreasing denial (Cox \& Price, 1990) by being used as an outlet for communication (Moores, 1983) and reducing client's shame (Johnson, 1990).

Music was also seen as a helpful intervention. For some, a song was found to convey far more about participants' internal dialogue than words. This supports the findings that song writing and lyric analysis relate to a positive emotional change in therapy (Baker et al., 2007, Jones, 2005). The use of creative modalities, such as music and art have also been suggested as ways to process early childhood drama in an attempt to heal the shame of the 'inner child'. This is largely based on the belief that painful childhood memories associated with shame are preverbal in origin and not represented by words (Wilson, 2012). Trauma and shame underpinned many of the narratives in the current research, and it was clear how paralysing it was for the women in the present study to access feelings that had remained buried for some time. 
Helpful and unhelpful processes in psychological therapy for female substance users

Despite the identification of music, art and writing as helpful tools in psychological therapy, there is not enough comprehensive meta-analysis to establish them as evidence based practices in the treatment of SUD suggesting further research is needed. Future treatment models should consider incorporating creative interventions into their therapeutic practice, as the creation of imagery has been found to enhance internal motivation to change for clients with SUD (Holt \& Kaiser, 2009).

Accessing and communicating feelings emerged as important, and avoiding feelings was seen by the women as a potential catalyst to relapse. Hence, the importance for a therapist to find interventions that enable clients to express themselves was recognised. Affect theorists have argued that addiction begins as 'sedative scripts' that buffer people from their shame and other negative feelings (Kaufman, 1996). When using substances, users either gain the relief of feeling 'normal' or gain a feeling of superiority, such that the sedating substance becomes linked to their emotional distress (Kaufman, 1996). Without drugs to mediate their feelings, participants talked about the expectation to talk in sessions as "scary" and "massive". Hence, finding another language to access and communicate feelings was so important in keeping them safe. Given that the statistics show that half of all clients with SUD fail to return after the second session, it is worth exploring how therapists can improve early engagement by utilising the findings from this research.

Incorporating creative interventions enabled these women to open up. Without that process, the internalisation of negative emotions may have caused them to revert back to old behaviours. Research on working with clients with SUD has placed importance on identifying the client's core beliefs, as it is their pain and shame that ignite their need to stay defended (Canning, 1999). As most SUD clients think they are 
Helpful and unhelpful processes in psychological therapy for female substance users

unlovable and worthless, psychological therapy has to displace those feelings of shame to increase their self-esteem (Canning, 1999).

Shame has been reported as playing a central role in SUD as it disrupts the natural functioning of the self, leading to low self-esteem, insecurity and inferiority (Kaufman, 1996). Shame is also part of the normal phenomenology of addiction and, conversely, can be a motivation towards change (Owen, 2013). Shame was not explicitly addressed as a separate theme in the analysis as it emerged throughout the extracts and impacted all the themes. Working with a client to process this feeling is seen as crucial to the recovery process as well as a way of establishing intimacy in the relationship. An implicit example of the shame is demonstrated by the way the women talked about not wanting to share their drug using behaviours, as it had taken them to the "depths of despair" (Louise, 247) and "left me feeling ashamed" (Anita, 654). Therefore, a clinical implication of this study would be to place a focus on how to actively de-shame clients in the sessions. There has been extensive theorisation about shame, but much less research on working with this emotion in therapy (Tangney\& Dearing, 2011). Normalising a client's experience, externalising the voice of shame, and validating this feeling through fostering a relationship with the therapist, could all be ways of improving engagement of SUD clients.

This concept of shame was also evident in the second superordinate theme 'Identification with my therapist' and how it was overcome when they felt understood in the psychological process. This was intimately linked to a second important finding, pertaining to clients' ideas about how similar and different they were to the therapist. If the women perceived or believed that their therapist was also in 'recovery' from drug or alcohol addiction, this had a positive impact on their psychological relationship. It 
Helpful and unhelpful processes in psychological therapy for female substance users

instilled hope about their own recovery and enabled the women to feel more understood. A counsellor that 'had been there, done that,' meant that past shameful behaviours could be addressed, without judgement. At the same time, difference was equally important, boundaries and maintaining the role of 'therapist' was also needed, as this helped participants to feel their respective therapists could contain and hold them in sessions, as well as provide a space for them to succeed themselves. These women recognised that they were in a professional relationship with their therapist, but, because of this shared connection, their interpersonal relationship felt closer than the realms of a normal therapeutic relationship.

This research does not suggest that all clinical practitioners working with SUD clients need to have, or have had, the same medical diagnosis to work with this client group effectively, but to allow the potential for identification. The most helpful aspect of therapy, for these participants, was the perception that they were in some way the same as their therapist. The belief that they felt understood, not judged and not alone in their journey was important to them. This illustrates the strength of peer support and working with service users, potentially combined with a counselling approach. These humanistic qualities underpin the field of counselling psychology, but this research suggests learning more about what underpins the client's addiction and having a greater knowledge of AA is needed. As AA is about addicts supporting other addicts and the spiritual intervention of total abstinence, clinicians who have no experience of addiction could integrate this approach into their treatment plans, and therapists in 'recovery' could convey their knowledge to make the client feel they were understood.

Despite the positive impact that the perception of being "similar" had on the women in this study, research is varied on how effective a counsellor in recovery 
Helpful and unhelpful processes in psychological therapy for female substance users

actually is. It has been found that counsellors in this position can offer an understanding unmatched by those that have never experienced addiction, but there is also a risk of imposing their own beliefs upon the client, resulting in a dissolution of appropriate boundaries (Manejwala, 2014). A therapist in this position may also replicate their own treatment experience, instead of identifying their client's individual needs. In order to avoid these pitfalls, an effective therapist needs to maintain boundaries and to be alert to the possibility of unconscious collusion, in which the real issues become avoided (Manejwala, 2014).

Counselling psychologists may embrace the idea of connecting with their client, as humanistic approaches argue that similarity, in terms of both the patient and therapist being genuine and authentic, is itself a tool for effectiveness (Rogers, 1951). They argue that self-disclosure and therapists' transparency as the most important factor in predicting clinical outcome (Norcross and Goldfried, 1992). Recent psychoanalyticpsychodynamic literature also posits that strict neutrality is impossible to maintain and that revealing an authentic self actually also enhances the therapeutic experience (Bloomgarden \& Mennuti, 2009; Knox \& Hill, 2003). However, ethically, the intent of the therapist has to be considered, as disclosure should be focused on the client's welfare and not fuelled by the gratification of the therapist's needs (Bridges, 2001, Zur, 2007). Another concern is that the therapist burdens the client or creates a situation in which the client needs to care for the therapist (Fisher, 2004). Therefore, these considerations should be monitored before disclosure takes place.

This study has revealed the positive impact these women felt at having a therapist they could relate to, which links to the Najavitis et al. (2000) study on the strength of the therapeutic alliance, and Waters et al. (2014) study on attachment. The 
Helpful and unhelpful processes in psychological therapy for female substance users

Waters et al. (2014) study was the only previous qualitative study identified that explored the subjective experiences of clients, albeit in recovery. Waters al al. (2014) focussed on cumulating profiles for each participant and by exploring the dominant narratives provided by participants, found attachment as the main theme. The client's secure attachment to their psychologists led to strengthening their internal working model, enabling them to develop positive beliefs about their therapist's intentions towards them. In my research, the womens' implicit attachment to their therapist was also helpful in their recovery, yet something that was slow to develop. The women talked about their initial fear of trusting their counsellor and concern that their feelings would not be contained, yet through the process of identification, their attachment shifted significantly with the knowledge, or perception, that their counsellor could also be an addict.

This leads to the final superordinate theme 'Getting towards acceptance.' This places a focus on how these womens' attitudes transitioned from resistance to acceptance within psychological therapy. The narratives revealed that the intersubjective relationship, overcoming negative emotions and a shift in their perception of themselves as an addict, underpinned their transformation. This illustrated how the psychological process had to encompass each theme for this transition to occur. The literature review revealed that a transformation in identity was key to sustaining a client's recovery. However, it also highlighted how clients feel conflict in relation to their new sense of self (Smith \& Rodriguez, 2014). This conflict was reflected in these women's narratives and their struggle to engage in therapy initially. Most research recognises how a client's perception of their identity from 'addict' to 'recovering addict' can be instrumental towards a successful outcome (Schaffer, 2014) but no previous research discusses how clients make that transition. 
Helpful and unhelpful processes in psychological therapy for female substance users

\subsection{Evaluation of this Study}

This section evaluates the Interpretative Phenomenological Analysis methodology used in this study, and its potential limitations in addressing the research question. I will provide a section on the clinical and research implications for Counselling Psychology and personal reflections. It will conclude with suggestions for future research.

\subsection{A Critique of Interpretative Phenomenological Analysis}

IPA is centrally concerned with experience, language and cognition, and questions whether a participant's experience can ever actually be fully understood. Language is important, as necessary tools to expressing and convening experience. However, Willig (2003) contends that language in fact constructs, rather than describes experience. The same person can potentially describe the same event in a number of ways, using different words each time, which means that words do not only express the experience but also shape it. Therefore, the range of constructions limits a person's ways of being or of viewing the world; every time different words are used, the meaning conveyed is altered. Therefore, direct access to someone's experience is arguably not possible, it depends on how a narrative is conveyed.

Participants in this study used reflections and descriptions to recall their psychological experience. Recruitment was purposefully targeted to participants who would be able to communicate their psychological experience in a language that I, the researcher, would be able to understand, acknowledging the co-construction of meaning of IPA (Smith et al., 2009). This study might not have worked if participants were unable to engage in this way. Clients in recovery from SUD often use the language 
Helpful and unhelpful processes in psychological therapy for female substance users

of AA (such as 'rock bottom,') and I was fortunate that my clinical work in the field meant I was familiar with these sorts of terms. It could be argued that the participants who chose to participate in this study were representative of a group that already had a relative ease talking about their experience of addiction and their behaviours. Inevitably, a different sample may have produced different accounts. A potential flaw could be that the meanings are always subjective to that client group and the researchers' interpretations, as understood through the double hermeneutic. However, the strength of IPA is the in-depth analysis gathered from this subjective approach.

A criticism of the snowballing recruitment technique used in this study was the risk that the women discussed their interviews with other recruits, biasing the results. Ideally, the treatment manager should have contacted each person first hand directly, enabling each person to be independent of other study participants. However, this vulnerable client group is hard to access and may not have agreed to participate if contacted in this way. However, this difficulty may have given another perspective to the findings. There is also the possibility that the women may have known each other through support networks, such as NA or AA, and not disclosed this information even if I had taken the direct approach. Therefore, acknowledging its limitations, this recruitment tool was deemed the best fit.

The role of cognition has also been an area of discussion with respect to IPA. Willig (2001) argues that IPA'S focus with cognition is incompatible with some aspects of phenomenology. The part concerned with non-propositional, pre-cognitive knowledge and ideas on the margin of consciousness Willig (2001). Conversely, Smith et al. (2009) view significant experience, meaning instances that provoke conscious 
Helpful and unhelpful processes in psychological therapy for female substance users

thought, as the main focus of IPA. Smith et al. (2009) have responded to Willig's concerns by discussing the layers of reflection produced when a participant engages with a researcher. These range from pre-reflection, the informal or intuitive reflection that happens spontaneously in everyday life, to formal or phenomenological reflection. Thus, everyday cognition is the subject of IPA.

During the interviews, participants recalled their psychological therapy by reflecting on helpful and unhelpful aspects in a way that represented how far they had come in terms of growth and self-awareness. In their descriptions, they talked about something they struggled with and followed this with a discussion of how they overcame the issue. It can be argued that any experience discussed during the interview would be a past experience, unless it was happening at that very moment. In this case, a possible limitation of IPA, is whether the amount of time that had passed since the participants had completed therapy can make a difference to what they recall. It is hard to distinguish between experience and the memory of experience. A potential implication stemming from this, is the re-conceptualisation of past and present experience. However, as IPA is about hot and cold cognition, hot meaning the immediate, unreflected thought and cold reflecting on the experience, there is no such thing as what happened, only interpretation of it from different perspectives. Therefore, no one is more accurate than the other. Since IPA's main focus is on the hermeneutic cycle and the way the researcher makes sense of the participant's meaning making, arguably the subject of the present analysis was the meaning each participant made of their respective experiences during the interview.

When participants spoke about their therapy, they selected the processes they found helpful and unhelpful from a position of superiority as the majority fail to 
Helpful and unhelpful processes in psychological therapy for female substance users

complete psychological treatment. Having participants that have been successful in reaching abstinence from their addiction might be different from the wider group of people looking for assistance with SUD who are unable to meet their own objectives. Therefore, this could be regarded as a limitation when recommending future practices for this client group.

Finally, the exclusion of comorbidity in this research may also be seen as a limitation, as the women were not asked to disclose if they had further mental heath conditions. Psychosis was the only exclusion criteria. Despite the high probability that other orders did exist, using the IPA approach meant focusing solely on the subjective meaning that the women ascribed to psychological therapy for SUD and their perceptions of what they deemed helpful and unhelpful. There was a fear that discussing other conditions would digress their focus away from the title. From the transcripts, the data that emerged showed depression and anxiety did feature, yet were not explicitly confirmed by the women as diagnosed conditions. Exploring how a dual diagnosis influences the process of psychological therapy would be useful for future studies. However, the findings that emerged in this IPA study emphasised the importance of the relationship, interventions and their own shift in attitude over time, regardless of their medical diagnosis.

\subsection{Clinical and Research Implications for Counselling Psychologists}

Counselling psychology has origins in phenomenological, existential and humanistic thinking that promotes the view that human beings should be seen in a holistic manner (Strawbridge and Woolfe, 2003). Hence, the study is relevant to 
Helpful and unhelpful processes in psychological therapy for female substance users

counselling psychology as it sheds light on how these individuals constructed meaning out of the process of psychological therapy, and the aspects of psychological therapy they found helpful and unhelpful. Fairfax (2008) highlights how one of the distinguishing features in counselling psychology is the attention applied to the therapeutic process and phenomenological understanding of the client, rather than a diagnostic manual such as the DSM-V. Since the therapeutic relationship is central to the findings in this thesis, a number of clinical and research implications can be suggested.

This research highlights how SUD users appear to require a very particular type of structure and identification within psychological therapy that has not been previously explored in the literature. The analysis has shown the subtle interplay between similarity and difference within the relational that could be actively drawn upon by therapists working with this client group. From the participants' accounts, the ability to conceive this idea of similarity and difference at once was what felt transformative in the therapeutic process. These participants needed to feel understood, feel listened to, and feel that their counsellor had been there and experienced similar behaviours; while also feeling that their therapist wanted the relationship with them. If the therapist had not experienced similar behaviours, then at least hold knowledge of addiction that the client could perceive them as informed and feel understood. At the same time, the participants needed to know that their therapist was bound by professional ethics, that they might or might not care about them, and that they were different enough. When a client gets to a place where they can internalise these dynamics and hold both positions of similarity and difference, then it feels that progress in the therapy can be achieved. 
Helpful and unhelpful processes in psychological therapy for female substance users

Another clinical and research implication that emerged from this thesis was the concept of shame. 'Shame operates everywhere in therapy because clients are constantly concerned about what part of their inner experience can be revealed safely and what part must be kept hidden' (Greenberg and Palvio, 1997, p.38). Whilst I was able to sense a significant amount of shame in the narratives, the participants did not speak directly about it, as mentioned previously. Clinically, it has been recognised that clients and clinicians struggle to work with shame (Tangney \& Dearing, 2001), however, if it remains unspoken, then it can have detrimental consequences to the individual.

From the experiences participants shared in this study, it is clear, that clinicians working with SUD clients need to be attuned and responsive to non-verbal and verbal indicators of shame as it can directly impact engagement. The therapeutic process itself may be seen as shame inducing, as clients are required to open up their inner thoughts that may have remained hidden. Clients with a history of 'failed attempts' at therapy for their addiction may also find this a source of shame. For a number of the women, this was not their first therapeutic attempt at coming off drugs. With such high failure rates, repeated episodes of treatment could maintain that shame and sense of failure. Clinically, therapists need to be aware around the ways in which shame may impact engagement in the therapy. The relational stance towards this feeling could potentially be the difference between retaining a client or losing them after the second session.

The women's accounts described how their resistant and defensive behaviour was overcome through the relational way their therapist worked. One of the ways shame could be dealt with in therapy is by making the client feel that the therapist is beside them in the process. Validating and normalising their feelings and offering an empathic, 
Helpful and unhelpful processes in psychological therapy for female substance users

affectively attuned relationship could help regulate the shame that client's experience, and transform this emotion into an internal resource that will keep clients safe.

\subsection{Suggestions for Future Research}

Some suggestions for future research have been noted throughout the discussion. Here, I propose some specific ideas inspired by this research and the present findings. Future clinical research could include:

1. Approaching clients with SUD who have left psychological therapy after two sessions, to find out what was unhelpful and the reason they left.

2. Find out whether there is a correlation between therapist self-disclosure and therapeutic outcomes for clients with SUD.

3. Interview women directly about therapist disclosure and undertake a quantitative study on how they perceived the similarity and difference between themselves and their therapist.

4. Use art and music in the actual research process for further exploration into helpful and unhelpful factors for SUD clients in psychological therapy for participants that struggle to engage verbally.

5. Explore how important it is for clients to know if their therapist is in 'recovery' and how it impacts psychological process.

6. Explore the relational perspective of working with SUD clients can be explored further. A potential direction would be to look at how to foster early engagement and de-shaming techniques.

\subsection{Personal Reflections}


Helpful and unhelpful processes in psychological therapy for female substance users

In this section, I provide some final reflections on my research, by way of inviting the reader into my experience as a researcher. I acknowledge that in this research, my influence as a researcher, a trainee counselling psychologist, an addictions counsellor and a person, was an inevitable aspect of the interpretive process. The choice of research subject, research question, method of data collection, interviews and analysis have all been influenced in some way by me (Harper, 2003). Personally, my clinical training in addiction, combined with my personal experiences, have all led to my interest in learning about womens' subjective experience of psychological treatment for SUD. From a poststructuralist perspective, the researcher's influence is unavoidable and seen to exist regardless of the method or methodology (Finlay \& Gough, 2003).

One of my greatest concerns when I started to engage with the data was ensuring that my experience would not impose on that of the participants'. In my attempts to safeguard their experience, especially in the early stages of this study, I felt insecure about whether I would be able to give justice to the women's perspectives. However, as the research process evolved, I realised that my interpretation was about building on their narrative and I inevitably gained insight into my own processes of meaning making.

Reflecting on my initial resistance to interpretation, I came to understand that I was trying to find and clarify my role as the researcher, and manage that with my own experience of being a trained addictions counsellor. Being a novice in IPA meant that everything was new; all the concepts had to be understood and assimilated from the beginning. It took time and effort for me to feel more comfortable with phenomenological inquiry. 
Helpful and unhelpful processes in psychological therapy for female substance users

To some degree, I harboured the personal desire to reach some truth or theory about psychological therapy for SUD, as it does affect such a vast number of people. However, as a social constructivist, I recognised that I was responsible for providing research that relayed the participants' accounts as accurately as possible. In practice I was terrified that I would get it wrong, that I would not be able to see what I was supposed to see and often I would repeat the process of reading and making notes, again and again, fearing I was missing something significant. In dialoguing with my participants, I would also add my clinical interpretations, trying to perhaps clarify the nature of interpretation itself.

Having worked with women with addictions, I had to consider my own values and responses in terms of interpretation, as I knew I felt passionate about helping this client group. I believe interpretation can translate into a claim for reality, and privileged access to underlying meaning. I did not feel however that I wanted to make such a claim and had to be careful to bracket my own reality and opinions. At the same time, I did recognise the importance of interpretation.

IPA calls for tentative interpretations, open in nature, that are meant to enrich that which is presented by noticing details, patterns and connections. This type of interpretation does not assume a position of expertise, or higher knowledge, and in that sense it is more difficult to achieve. It requires openness and devotion to the research material. Looking back, I feel comfortable with my role as the researcher who interpreted the data, in a tentative way, whilst acknowledging that the end result, (this research thesis), is a product of my meaning-making process.

This study represents a five-year journey, through research and clinical practice. My personal interest in the research topic was disclosed in the reflexivity section. 
Helpful and unhelpful processes in psychological therapy for female substance users

However, I could not have anticipated the ways in which my own therapeutic practice would be challenged by my understanding of what these women found helpful and unhelpful, and how I made sense of their stories. This process has challenged my previous approach to engagement, i.e. my expectation on the client to be able to talk and the effectiveness of a psychiatric diagnosis. In relation to my own therapeutic practice, I know I will focus on exploring different forms of self-expression with clients to open the channels of communication and visit recovery groups to increase my understanding of what underpins an addiction from various perspectives, rather than utilising my understanding from the role of friend, family member or addictions counsellor. I will also interpret resistance as a defence, rather than make an assumption the client is not ready to engage, a belief I might have held in the past.

My shifting and growing experience as a researcher has come to sit alongside my identity as a clinician, encompassing the scientist-practitioner stance that Counselling Psychology identifies with. Through the research process, I have been able to consider, slowly and systematically, the way that meaning-making occurs, the nature of interpretation and the importance of words. I have often wondered if the women in my research also have a new understanding of meaning around their own developmental journey.

In terms of clinical supervision, I often experienced a parallel process that may have reflected the feelings that emerged with this client group. In conducting interviews and writing the thesis, I was plagued with feelings of doubt and concern about whether I was doing things right. I wonder on reflection, whether this mirrored the feelings that the women had in the interviews and in therapy. A fear of "doing things right" and being accepted for who they were. I also wonder if this is a position other therapists 
Helpful and unhelpful processes in psychological therapy for female substance users

find themselves in at the beginning of psychological therapy with SUD clients, contributing to the low outcome success rates. This phenomenological research has raised a number of challenges in the relational field and this finding may also be a reflection of the difficulty faced by women engaging in the psychological process.

Supervision and personal therapy was also used to work through my feelings throughout this process around addiction. I recognised that the intersubjective stance that I took with the participants brought up feelings of empathy and admiration for this group of women. I felt so supportive of this client group and often wondered if the women picked up on these feelings in the transference, hence the predominant feedback on helpful factors within the process, rather than the unhelpful. Without being able to answer this, I can reflect that this study has convinced me to reflect on my knowledge and subjective experiences, and how I position myself as a researcher and counselling psychologist working with this client group. It has also enabled time to reflect on how I will work differently with SUD clients in the future. Overall, this thesis has been an emotional journey, but also a testament to my attempt to develop a greater understanding of myself as a person, and my relational approach as a clinician.

\subsection{Conclusion:}

Within this chapter, I have explored the strengths and limitations of this study and the challenges that emerged when conducting it. This thesis has offered in-depth insights into the experience of these women. As so few SUD clients are successful at completing psychological treatment, this original piece of work, provides rich data about what aspects of the therapeutic process contributed to their success. These findings can indicate new pathways for clinical practice and research, and raise 
Helpful and unhelpful processes in psychological therapy for female substance users

awareness of what the clients are struggling to bring in to therapy and how to overcome those obstacles.

This thesis has contributed to a greater understanding of women's subjective experience of psychological treatment for SUD. If we really want to improve services for women with addiction, attention needs to be paid to their subjective experience and the meanings attached to the process. IPA as an approach has been an appropriate method to do this.

In learning about what the women found helpful and unhelpful within their psychological process, practitioners can re-evaluate their knowledge and therapeutic practices when working with this client group. Among the recommendations for practice are working through resistant, defensive behaviours by validating feelings of shame and anger. Improve early engagement with clients by utilising alternative interventions to encourage self-expression and access feelings. Increase understanding and knowledge around addiction in order to strengthen the relational dynamic.

Lastly, I would like to dedicate the concluding words of this thesis to the eight participants that made it possible. These women opened up and shared deeply their experience of therapy, and in that process, provided an insight into their struggle and journey with addiction. The women expressed such gratitude for their psychological therapy that they wanted to share, in an open and honest approach, in the hope it may help others and as a way of giving back to the charity that helped them. Their original contribution also allowed them to represent a marginalized group in the literature, in the hope that further research will build on this research. These women all showed great courage, strength and determination to overcome their addiction and I hope that these findings will enable others to emulate their success. 
Helpful and unhelpful processes in psychological therapy for female substance users

\section{References}

ABC Money (2017). The Financial cost of Addiction. Retrieved October 162017 from http://www.abcmoney.co.uk/2017/05/22/the-financial-cost-of-addiction/

Alcoholics Anonymous World Services. (AA). (1972). A brief guide to Alcoholics Anonymous. New York: Author.

Alcoholics Anonymous World Services. (2007). A.A. at a glance. Retrieved March 10, 2016 from http://www.aa.org.

Aletraris, L., Paino, M., Edmond., M. B., Roman., P. M., \& Bride, B. E. (2014). The Use of Art and Music Therapy in Substance Abuse Treatment Programs. Journal of Addictions Nursing, 25(4), 190-196. http://doi.org/10.1097/JAN.0000000000000048

Allen, J. P., \& Wilson, V. B. (Eds.). (2003). Assessing alcohol problems: A guide for clinicians and researchers (NIH Publication No. 03-3745) (2nd ed.). Bethesda, MD: U.S. Department of Health and Human Services, National Institutes of Health, National Institute on Alcohol Abuse and Alcoholism.

Allen, K. (1994). Development of an instrument to identify barriers to treatment for addicted women from their perspective. International Journal of Addictions, 29(4), 429-444.

Allport, G. (1940). The psychologist's frame of reference. Psychology Bulletin, 37:128.

American Psychiatric Association. (2013). Diagnostic and statistical manual of mental disorders (5th ed.). Washington, DC

Arfken, C, L., Klein, C., DiMenza, S., Shuster, C, R. (2001). Gender differences in problem severity at assessment and treatment retention. Journal of Substance Abuse Treatment 20:53-7. 
Helpful and unhelpful processes in psychological therapy for female substance users

Ashley, O.S., Marsden, M.E., \& Brady,T.M. (2003) Effectiveness of substance abuse treatment programming for women. Journal of Drug Alcohol Abuse. ;29:19-53. doi: 10.1081/ADA-120018838.

Back, S. E., Dansky, B.S., Carrol, K.M., Foa, E.B., \& Brady, K.T. (2001) Exposure therapy in the treatment of PTSD among cocaine-dependent individuals. Journal of Substance Abuse Treatment, 21 (1), 35-45

Baker, F., Gleadhill, A., Libby, M., \& Dingle, G.A. (2007). Music therapy and emotional exploration: exposing substance abuse clients to the experiences of nondrug induced emotions. The Arts in Psychotherapy. Vol 34 (4), 321-330.

Bawor M., Dennis J, Varenbut M. (2015) Sex differences in substance use, health and social functioning among opioid users receiving methadone treatment: a multicenter cohort study. Biology of Sex Difference. (6):21

Beck, A. (1976) Cognitive Therapies and the Emotional Disorders, New York: International Universities Press

Biernacki, P. (1986) Pathways from Heroin Addiction: recovery without treatment. Temples University: Philadelphia.

Blass, R. B. (2015). Conceptualizing splitting: On the different meanings of splitting and their implications for the understanding of the person and the analytic process. International Journal of Psychoanalysis, 96(1), 123-139. doi:10.1111/ 17458315.12326

Bloom, B., Owen, B., \& Covington, S. (2004). Gender responsiveness: Research, practice and guiding principles for women offenders. Washington DC: National Institute of Corrections, US Department of Justice.

Borrill, J., Fox, P., \& Roger, D. (2011). Religion, ethnicity, coping style, and selfreported self- harm in a diverse non-clinical UK population. 14(3), 259-269. doi:10.1080/ 13674670903485629

Brady, K.T., Back, S.E., Greenfield, F.S., (2007) Women and Addiction: A Comprehensive Handbook. Guilford Press. London 
Helpful and unhelpful processes in psychological therapy for female substance users

Brady, K.T., Grice, D.E., Dustan, L., \& Randall, C. (1993). Gender differences in substance use disorders. American Journal of Psychiatry, 150, 1707-1711

Bramley, N,. \& Eatough, V. (2005). An idiographic case study of the experience of living with Parkinson's disease using interpretative phenomenological analysis.

Psychology \& Health, 20:223-235.

British Psychological Society. (2009). Code of ethics and conduct . Retrieved: August 2016, from http:/ / www.bps.org.uk/ what-we-do/ ethics-standards/ ethics-standards

Bridges, N. A. (2001). Therapist's self-disclosure: Expanding the comfort zone. Psychotherapy, 38, 21-30

Brocki J, M., \& Wearden, A, J. (2006). A critical evaluation of the use of interpretative phenomenological analysis (IPA) in health psychology. Psychology and Health Volume 21. Issue 1.

Brook, J. A. (1992). Freud and Splitting. International Review of Psycho-Analysis, OO(19), 335-350

Buckingham, S., Frings, D., \& Albery, I. P. (2015). Group membership and social identity in addiction recovery. Psychology of Addictive Behaviors, 27(4), 1132-1140.

Carroll, K.M., Libby, B., Sheehan, J., Hyland, N. (2001). To enhance treatment initiation in substance abusers: an effectiveness study. American Journal of Addictions, 10, 335-339.

CJS, (2013). No quick fix: exposing the depth of Britain's drug and alcohol problem. Retrieved October14,2016. From: http://www.centreforsocialjustice.org.uk/library/noquick-fix-exposing-depth-britains-drug-alcohol-problem

Clinical Guidelines on Drug Misuse and Dependence Update 2017 Independent Expert Working Group (2017) Drug misuse and dependence: UK guidelines on clinical management. London: Department of Health. Retrieved November 6, 2017. From: 
Helpful and unhelpful processes in psychological therapy for female substance users

https://www.gov.uk/government/uploads/system/uploads/attachment_data/file/628634 /clinical_guidelines_2017.pdf

Compton,W.M.,Thomas,Y,F., \& Stinson F,S. (2007). Prevalence, correlates, disability, and comorbidity of DSM-IV drug abuse and dependence in the United States: results from the National Epidemiologic Survey on Alcohol and Related Conditions. Archive General Psychiatry. 64:566-76

Cooper,M., \& McLeod, J. (2011). Pluralistic Counselling and Psychotherapy. London. Sage.

Covington, S. (2008). Helping Women Recover: A Program for Treating Addiction. San Francisco: Jossey-Bass.

Covington, S. 2004. Voices: A Program of Self-Discovery and Empowerment for Girls. Carson City, NV: The Change Companies.

Crossley, M.L. (2000). Introducing Narrative Psychology: Self, Trauma and the Construction of Meaning. Buckingham: Open University Press.

Culbreth, J. (2000). Substance abuse counsellors with and without a personal history of clinical dependency: a review of the literature. Alcoholism Treatment Quarterly, 18(2): 67-82.

Division of Counselling Psychology. (2005). Professional practice guidelines.

Leicester: British Psychological Society. Retrieved 3 July 2016, from www.bps.org.uk/publications/policy-guidelines/practice-guidelines-policydocuments/practice-guidelines-policy-docum

Denzin, N. (1995). 'Symbolic Interactionism', in J.A. Smith, R. Harre and L. Van Langenhove (eds). Rethinking Psychology. London: SAGE. 43-58.

De Weert-Van Oene, G.H., Schippers, G.M., De Jong, C.A.J., \& Schrijvers, G.J.P. (2001). Retention in substance dependence treatment: The relevance of in-treatment factors. Journal of Substance Abuse Treatment, 20, 253-261 
Helpful and unhelpful processes in psychological therapy for female substance users

Dolan, M., \& Völlm, B. (2009). Self-harm among UK female prisoners: A crosssectional study. Journal of Forensic Psychiatry \& Psychology, 20(5), 741-751. doi:10.1080/ 14789940903174030

Duongsaa, U. (1998). Women, Gender and Drugs. Speech at the United Nations General Assembly. New York.

Dutra, L., Stratopoulou, G., Shawnee, M., Basden, L., Powers, M., \& Otto, M. (2008). A meta-analytic review of psychosocial interventions for substance use disorders. American Journal of Psychiatry. Vol. 165. No.2 p. 179-187

Eatough, V., \&Smith, J. A. (2008). Interpretative phenomenological analysis. The SAGE handbook of qualitative research in psychology. SAGE publications.

Elliott, R. (1985). Helpful and non-helpful events in brief counselling interviews: An empirical taxonomy. Journal of Counselling Psychology, 32, 307-322.

Elliot, R. (2010). Psychotherapy change process research: Realising the promise. Psychotherapy Research, 20 (2), 123-135.

Elliott, R., Fischer, CT., and Rennie, DL. 1999. Evolving guidelines for the publication of qualitative research studies in psychology and related fields. British Journal of Clinical Psychology, 38: 215-229

Elliott, R. \& Shapiro, D.A. (1992). Client and Therapist as Analysts of Significant Events. In Toukmanian, S.G. \& Rennie, D.L. (eds.), Psychotherapy Process Research: Paradigmatic and Narrative Approaches London: Sage Publications.

EMCDDA (2017). The European Monitoring Centre for Drugs and Drug addiction. Accessed 20 October 2017 from: http://www.emcdda.europa.eu/data/stats2017

Ettore, E. (1992). Women and substance use/abuse: towards a feminist analysis or how to make dust fly. Women's Studies International Forum, 12, 593-602

Fairfax, H. (2008). CBT or Not CBT, is that really the question? Counselling Psychology Review, 23(4): 27-35. 
Helpful and unhelpful processes in psychological therapy for female substance users

Finlay, L. (1999). Applying phenomenology in research: Problems, principles and practice. British Journal of Occupational Therapy, 62(7), 299-306.

Finlay, L. (2002). Negotiating the swamp: the opportunity and challenge of reflexivity in research practice. Qualitative Research. Vol 2. Issue 2. 209-230.

Finlay, L. (2006). The body's disclosure in phenomenological research. Qualitative Research in Psychology, 3(1), 19-30. doi:10.1191/1478088706qp051oa

Finlay, L. (2008). A dance between the reduction and reflexivity: Explicating the "phenomenological psychological attitude". Journal of Phenomenological Psychology, 39(1), 1-32. doi:10.1163/ 156916208X311601

Finlay, L. (2003). Through the looking glass: intersubjectivity and hermeneutic reflection. In L. Finlay \& B. Gough (Eds.) Reflexivity: A Practical Guide for Researchers in Health and Social Sciences. Blackwell: Oxford.

Fisher, C. D. (2004). Ethical issues in therapy: Therapist self-disclosure of sexual feelings. Ethics and Behavior, 12, 105-121.

Fonagy, P., \& Target, M. (2003). Psychoanalytic Theories: Perspectives from Developmental Psychopathology. Abingdon: Routledge.

Fonagy, P. (2015). The effectiveness of psychodynamic psychotherapies: An update. World Psychiatry, 14(2), 137-150. http://doi.org/10.1002/wps.20235

Forrester, M. A. (2013). Doing Qualitative Research in Psychology. A practical Guide. London. SAGE.

Frances, A. (2013). Saving normal: An insider's revolt against out-of-control psychiatric diagnosis, DSM-5. Big Pharma, and the medicalization of the ordinary life. New York. William Morrow.

Friedmann, P. D., Lemon, S. C., Stein, M. D., \& D'Aunno, T. A. (2003). Accessibility of addiction treatment: Results from a national survey of outpatient substance abuse treatment organizations. Health Services Research, 38(3), 887-903. 
Helpful and unhelpful processes in psychological therapy for female substance users

Frost, N. (2011). Qualitative research methods in psychology. Maidenhead: McGrawHill Open University Press.

Georgakopoulou, A., \& Goutsos, D. (1997). Discourse analysis: An introduction. Edinburgh: Edinburgh University Press.

Giorgi, A. (2002). The question of validity in qualitative research. Journal of Phenomenological Psychology, 33(1), 1-18. doi:10.1163/156916202320900392

Giorgi, A. (2008). Difficulties encountered in the application of the phenomenological method in the social sciences. Indo-Pacific Journal of Phenomenology, 8(1), 1-9.

Giorgi, A. (2011). IPA and science: A response to Jonathan Smith. Journal of Phenomenological Psychology, 42(2), 195-216. doi:10.1163/156916211X599762 Glucklich, A. (2001). Sacred pain: Hurting the body for the sake of the soul. New York: Oxford University Press.

Greenfield, S. F., Back, S. E., Lawson, K., \& Brady, K. T. (2010). Substance Abuse in Women. The Psychiatric Clinics of North America, 33(2), 339-355. http://doi.org/10.1016/j.psc.2010.01.004

Greenfield, S. F., \& Grella, C. E. (2009). What Is "Women-Focused" Treatment for Substance Use Disorders? Psychiatric Services (Washington, D.C.), 60(7), 880-882. http://doi.org/10.1176/appi.ps.60.7.880

Grella, C. E., \& Greenwell, L. (2004). Substance abuse treatment for women: Changes in the settings where women received treatment and types of services provided, 1987-1998. Journal of Behavioral Health Services \& Research, 31(4), 367383.

Grella, C. E. (2008). From generic to gender-responsive treatment: changes in social policies, treatment services, and outcomes of women in substance abuse treatment. Journal of Psychoactive Drugs. 327-43.

Greenberg, L., \& Paivo, S. (1997). Working with Emotions in Psychotherapy, New York: The Guildford Press 
Helpful and unhelpful processes in psychological therapy for female substance users

Gossop, M. (2004). Drug Addiction and it's Treatment. Oxford University Press.

Gossop M., Manning V., \& Ridge, G. (2006). Concurrent use of alcohol and cocaine: differences in patterns of use and problems among users of crack cocaine and powder cocaine. Alcohol and Alcoholism, 41(2):121-125.

Gossop, M., Marsden, J., Stewart, D., Kidd, T. (2002). Changes in use of crack cocaine after drug misuse treatment: 4-5 year follow-up results from the National Treatment Outcome Research Study (NTORS). Drug and Alcohol Dependence, 66, 21-28.

Gossop, M., Marsden, J., Stewart, D., Kidd, T. (2003). The National Treatment Outcome Research Study (NTORS): 4-5 year follow- up results. Addiction, 98, 291303.

Hanninen, V., \& Koski-Jannes, A. (1999). Narratives of recovery from addictive behaviours. Addiction, 94, 1837-1848.

Halling, Steen (2008). Embodied inquiry: Phenomenological touchstones for research, psychotherapy and spirituality. Journal of Phenomenological Psychology 39 (2):241243.

Hasin, D.S.,Stinson F, S., Ogburn, E., Grant, B. F. (2007). Prevalence, correlates, disability, and comorbidity of DSM-IV alcohol abuse and dependence in the United States: Results from the National Epidemiologic Survey on Alcohol and Related Conditions. Archives of General Psychiatry. 64:830-842

Heidegger, M. (1962). Being and time. Blackwell. Oxford.

Hernandez-Avila, C. A., Rounsaville, B.J., Kranzler, H. R. (2004). Opioid-, cannabis and alcohol-dependent women show more rapid progression to substance abuse treatment. Drug Alcohol Depend.74(3):265-72

Hertz, R. (Ed.). (1997). Reflexivity and voice. Thousand Oaks, CA: Sage. 
Helpful and unhelpful processes in psychological therapy for female substance users

Hill, J., \& Leeming, D. (2014) 'Reconstructing 'the alcoholic': Recovering from alcohol addiction and the stigma this entails. International Journal of Mental Health and Addiction. ISSN 1557-1874

Hodge, D., \& Lietz, C.A. (2014). Using spiritually modified cognitive behavioural therapy in substance dependence treatment: Therapists' and clients' perceptions of the presumed benefits and limitations. Health Social Work, 39 (4): 200-210.

Holt, E., \& Kaiser, D.H. (2009). The First Step Series: Art therapy for early substance abuse treatment. The Arts in Psychotherapy. 36:245-250.

Howitt, D. (2010). Introduction to qualitative methods in psychology. Harlow: Pearson.

Hulstrand, Karen L., "Shame - the Good, the Bad and the Ugly: Therapist Perspectives" (2015). Master of Social Work Clinical Research Papers. Paper 459. http://sophia.stkate.edu/msw_papers/459

Humphreys, K., Moos, R.H., 2001. Can encouraging substance abuse patients to participate in self-help groups reduce demand for health care? A quasi- experimental study. Alcohol Clinical Research. 25, 711-716.

Husserl, E. (1925; trans. 1977). Phenomenology Psychology. The Hague. Martinus Niijhoff.

Ingram, R.E., \& Luxton, D. (2015). Vulnerability-stress models. Cited in Hankin, A (2005). Development of Psychopathology. Sage Publications.

Jhanjee, S. (2014). Evidence Based Psychosocial Interventions in Substance Use. Indian Journal of Psychological Medicine, 36(2), 112-118. http://doi.org/10.4103/0253-7176.130960

Johnson B. (1999). Three perspectives on addiction. J. Am. Psychoanalysis. Association. 47 791-815 10.1177/00030651990470031301

Kalant, H. (2010). Drug classification: science, politics both or neither? 105 (7) 11469. doi: 10.1111/j.1360-0443.2009.02830.x. 
Helpful and unhelpful processes in psychological therapy for female substance users

Kasket, E., \& Rodriguez, E.G. (2011). The identity crisis in trainee counselling psychology research. Counselling Psychology Review, Vol 26, No.4.

Kaskutas, L. A. (2009). Alcoholics Anonymous Effectiveness: Faith Meets Science. Journal of Addictive Diseases, 28(2), 145-157.

http://doi.org/10.1080/10550880902772464

Keegan, S. (2009). Qualitative research: Good decision making through understanding people, cultures and markets. London: Kogan Page.

Kelly, J.F., Yeterian, J.D., 2008. Mutual-help groups. In: O’Donohue, W., Cunningham, J.R. (Eds.), Evidence-Based Adjunctive Treatments. Elsevier, New York, pp. 61-105.

Kessler, R, C., McGonagle, K, A., \& Zhao, S., Nelson, C, B., Hughes, M., Eshleman S. (1994) Lifetime and 12-month prevalence of DSM-III-R psychiatric disorders in the United States. Results from the National Comorbidity Survey. Archives of General Psychiatry. 51:8-19.

Kutchins, H., \& Kirk, S.A. (1993). DSM-IV and the hunt for gold: A review of the treasure map. Research on Social Work Practice, 3, 219-235.

Koob, G. F. (2008). A role for brain stress systems in addiction. Neuron, 59(1), 11-34.

Koski-James, A. (2002). Social and personal identity projects in the recovery from addictive behaviours. Addiction Research and Theory. 10, (2), 183-202.

Kvale, S. (1996). Interviews: An Introduction to Qualitative Research Interviewing. California: Sage.

Kvale, S. (2007). Doing Interviews. Los Angeles. CA: Sage.

Langdridge, D. (2007). Phenomenological Psychology: Theory, Research and Method. Harlow: Pearson Education.

Langdridge, D. (2008). Phenomenology and critical social psychology: Directions and debates in theory and research.3, 1126-1142. doi:10.1111/ j.1751-9004.2008.00114.x 
Helpful and unhelpful processes in psychological therapy for female substance users

Larkin, M., Eatough, V., \& Osborn, M. (2011). Interpretative phenomenological analysis and embodied, active, situated cognition. Theory \&Psychology, 21(3), 318337. doi:10.1177/ 0959354310377544

Larkin, M., \& Griffiths, M. (2002). Experiences of addiction and recovery: the case for subjective accounts. Addiction, Research and Theory, 10, 281-311

Larkin, M., Wood, R., \& Griffiths, M.D. (2006). Towards addiction as a relationship. Addiction Research and Theory. (3). 207-215.

Larkin, M., Watts, S., \& Clifton, E. (2006). Giving voice and making sense in interpretative phenomenological analysis. Qualitative Research in Psychology, 3(2), 102-120. doi:10.1191/ 1478088706qp062oa

Laudet, A. B. (2007). What does recovery mean to you? Lessons from the recovery experience for research and practice. Journal of Substance Abuse Treatment, 33, $243-$ 256.

Laudet, A. B., \& White, W.L. (2008). Recovery capital as prospective predictor $\mathrm{f}$ sustained recovery, life satisfaction and stress among former poly-substance users. Substance Use and Misuse, 43,(1), 27-54.

Laudet, A. B., \& White, W. (2010). What are your priorities right now? Identifying service needs across recovery stages to inform service development. Journal of Substance Abuse Treatment, 38(1), 51-59

Lemma, A. (2003). Introduction to the Practice of Psychoanalytic Psychotherapy. Chichester : John Wiley and Sons.

Madill, A., Jordan, A., \& Shirley, C. (2000). Objectivity and reliability in qualitative analysis: realist, contextualist and radical constructionist epistemologies. British Journal of Psychology, 91: 1-20.

Manejwala, O. (2014). 'Should addiction counsellors disclose their recovery?. Addiction Treatment Methods. Retrieved January 10, 2017 from 
Helpful and unhelpful processes in psychological therapy for female substance users

http://www.rehabs.com/pro-talk-articles/should-addiction-counselors-disclose-theirown-recovery/

Martin, D., Garske, J., \& Davis, K. (2000). Relation of the therapeutic alliance with outcome and other variables: a meta-analytic review. Journal of Consulting and Clinical Psychology, 68(3): 438-450.

Matto, H.C. (2004). Applying an ecological framework for understanding drug addiction and recovery. Journal of Social Work and Practice in Addictions. 4 (3), 521.

McGraw, C. (2016). Six reasons why drug and alcohol rehab success rates are so low. Retrieved 31 October, 2017 from: http://www.theclearingnw.com/blog/6-reasonsdrug-alcohol-rehab-success-rates-are-so-low

McHugh, R. K., Hearon, B. A., \& Otto, M. W. (2010). Cognitive-Behavioral Therapy for Substance Use Disorders. The Psychiatric Clinics of North America, 33(3), 511525. http://doi.org/10.1016/j.psc.2010.04.012

McIntosh, J., \& McKeganey, N. (2001). Identity and recovery from dependent drug use: the addict's perspective. Drugs: Education, Prevention, and Policy, 8, 47-59.

McLeod, J. (2001). Qualitative Research in Counselling and Psychotherapy. London: Sage.

McLeod, J. (2003). Qualitative research methods in counselling psychology. In R.

Woolfe; W. Dryden; S. Strawbridge (Eds.) Handbook of Counselling Psychology. London: Sage.

McLeod, J. (2013). Developing Pluralistic Practice in Counselling and Psychotherapy: Using what the client knows. The European Journal of Counselling Psychology. Retrieved June 12, 2016 from http://ejcop.psychopen.eu/article/view/5

Mearns, D., \& Cooper, M. (2005). Working at Relational Depth, Sage: London.

Mendola, A., \& Gibson, R, L. (2016). Addiction, 12-step programs, and evidentiary 
Helpful and unhelpful processes in psychological therapy for female substance users

standards for ethically and clinically sound treatment recommendations: What should clinicians do? AMA Journal of Ethics. Volume 18, Number 6: 646-655.doi:

10.1001/journalofethics.2016.18.06.sect1-1606

McKeganey, N. (1995) Quantitative and Qualitative research in addictions: The unhelpful divide. DOI: 10.111/j.1360-0443.1995.tb02214.x. PubMed.

Miller, W. R., \& Rollnick, S. (2002). Motivational interviewing (2nd ed.). New York: Guilford Press.

Miller, P.G., Strang, J., \& Miller, P. M. (2010). Addiction research methods. Chichester: Wiley-Blackwell.

Milligan, K., Niccols, A., Sword, W., Thabane, L., Henderson, J., Smith, A., \& Liu, J. (2010). Maternal substance use and integrated treatment programs for women with substance abuse issues and their children: a meta-analysis. Substance Abuse Treatment, Prevention, and Policy, 5, 21. http://doi.org/10.1186/1747-597X-5-21

Moos, R.H., \& Moos, B.S. (2006). Participation in treatment and Alcoholics Anonymous: A 16 year follow up of initially untreated individuals. Journal of Clinical Psychology 62 (6), 735-750.

Moos, R., \& Timko, C. (2008). Outcome research on twelve-step and other self-help programs. In M. Galanter, \& H. O. Kleber (Eds.), Textbook of substance abuse treatment (4th ed. pp. 511-521). Washington, DC: American Psychiatric Press.

Murray, M. (2003). Narrative psychology and narrative analysis. In P. M. Camic, J. E. Rhodes \& L. Yardley (Eds.), (pp. 95-112). Washington, DC, US: American Psychological Association. doi:10.1037/ 10595-006

Murray, S. J., \&Holmes, D. (2014). Interpretive phenomenological analysis (IPA) and the ethics of body and place: Critical methodological reflections. Human Studies, 37(1), 15-30. doi:10.1007/ s10746-013-9282-0 
Helpful and unhelpful processes in psychological therapy for female substance users

Najavits, L.M., Crits-Christoph, P., \& Dierberger, A.E. (2000). Clinicians' impact on substance abuse treatment. Substance Use and Misuse. 35:2161-2190

National Treatment Agency (NTA). (2016). Official statistics. Retrieved July 2016, from http://www.nta.nhs.uk/statistics.aspx

Nathan, P.E., \& Skinstad, A.H. (1987).Outcomes of treatment for alcohol problems: current methods, problems and results. Journal of Consulting and Clinical Psychology. 55, 332-340.

National Institute on Drug Abuse, NIDA (2012). Principles of Drug Addiction Treatment: A Research-Based Guide (Third Edition). Retrieved December 1, 2016, from https://www.drugabuse.gov/publications/principles-drug-addiction-treatment$\underline{\text { research-based-guide-third-edition }}$

NIDA (2016). Media Guide. Retrieved May 2, 2017, from https://www.drugabuse.gov/publications/media-guide

Niv, Y., Joel, D., \& Dayan, P. (2006) A normative perspective on motivation. Trends in Cognitive Sciences 10(8):375-8

Neale, J., Allen, D., Coombes, L. (2005). Qualitative research methods within addiction. Addiction. 100, 1584-1593.

Nelson-Zlupko, L., Kauffman, E., Dore, M, M. (1995). Gender differences in drug addiction and treatment: implications for social work intervention with substanceabusing women. Social Work, 40:45-54

NTDMS (2016) Adult substance misuse statistics from the National Drug Treatment Monitoring System. Retrieved November 11, 2016, from

http://www.nta.nhs.uk/uploads/adult-statistics-from-the-national-drug-treatmentmonitoring-system-2015-2016[0].pdf

Norcross, J. C., \& Goldfried, M. R. (1992). Handbook of psychotherapy integration. New York: Basic Books. 
Helpful and unhelpful processes in psychological therapy for female substance users

Nordfjaern, T., Rundmo, T., Hole, R., (2010). Treatment and recovery as perceived by patients with substance addiction. Journal of Psychiatry and Mental Health Nursing. 17, 46-64

Orford, J. (2001). Excessive appetites: a psychological view of addictions. Wiley, Chichester, New York.

Osilla, K. C., Hepner, K. A., Muñoz, R. F., Woo, S., \& Watkins, K. (2009).

Developing an Integrated Treatment for Substance Use and Depression Using Cognitive Behavioral Therapy. Journal of Substance Abuse Treatment, 37(4), 412420. http://doi.org/10.1016/j.jsat.2009.04.006

Palmer, R. (1969). Hermeneutics, Evanston: Northwestern University Press

Parry, G., Shapiro, D. A. and Firth, J. (1986), The case of the anxious executive: A study from the research clinic. British Journal of Medical Psychology, 59: 221-223. doi:10.1111/j.2044-8341.1986.tb02687.x

Paulus, D. J., Bakhshaie, J., Lemaire, C., Garza, M., Ochoaperez, M., Valdivieso, J., Velasco, R.V., Bogiaizian, D., Kaufman, B., Robles, Z., Neighbors, C., Zvolensky, M.J. (2016). Negative affectivity and problematic alcohol use among Latinos in primary care: The role of emotion dysregulation. Journal of Dual Diagnosis. 12 (2), 137-147. doi: 10.1080/15504263.2016.1172897

Pearson, F., Pendergast, M, L., Podus, M., Vazan, P,. Greenwell, L,. Hamilton, Z. (2012) Meta-analyses of seven of the National Institute on Drug Abuse's principles of drug addiction treatment. Journal of Substance Abuse Treatment.

DOI:http://dx.doi.org/10.1016/j.jsat.2011.10.005

Peele, S. (2000). 'What Addiction is and is not: The Impact of Mistaken Notions of Addiction'. Addiction Research 8(6) p.599-607. Overseas publishers association.

Pilgrim, J. L., Dorward, R., \& Drummer, O. H. (2017) Drug-caused deaths in Australian medical practitioners and health-care professionals. Addiction, 112: 486493. doi: 10.1111/add.13619. 
Helpful and unhelpful processes in psychological therapy for female substance users

Polcin, D., Mulia, N., \& Jones, L. (2012). Substance Users' Perspectives on Helpful and Unhelpful Confrontation: Implications for Recovery. Journal of Psychoactive Drugs, 44(2), 144-152.

Ponterotto, J. G. (2005). Qualitative research in counseling psychology: A primer on research paradigms and philosophy of science. Journal of Counseling Psychology, 52(2), 126- 136. doi:10.1037/ 0022-0167.52.2.126

Prendergast, M, L., Podus, D., Chang, E., Urada, D. (2002). The effectiveness of drug abuse treatment: a meta analysis of comparison group studies. Drug and Alcohol Dependence, 67, 53-72.

Pringle, J., Drummond, J., McLafferty, E., \&Hendry, C. (2011). Interpretative phenomenological analysis: A discussion and critique. Nurse Researcher, 18(3), 20.

PROJECT MATCH Research Group (1997). Matching alcoholism treatments to client heterogeneity: Project Match post-treatment drinking outcomes. Journal of Studies on Alcohol, 58, 7-29.

Reid, K., Flowers, P., Larkin, M. (2005). Exploring lived experience. The Psychologist, 18 (1), 20-23.

Reid, K., Flowers, P., \& Larkin, M. (2005). Interpretative phenomenological analysis: An overview and methodological review. The Psychologist, 18: 20-23

Reicher, S., \& Taylor, S. (2005, September). Dialoguing across divisions:

Similarities and differences between traditions, The Psychologist, 18(9), 547-549.

Retrieved from

http://www.thepsychologist.org.uk/archive/archive_home.cfm?volumeID=18\&edition $\mathrm{ID}=127 \&$ ArticleID $=919$

Reis L., Eisner, M., Kosary, C., Hankey, B., Miller, B., Clegg, L., Edwards, B.(2003). SEER Cancer Statistics Review, 1975--2000. Bethesda, Maryland: National Cancer Institute. 
Helpful and unhelpful processes in psychological therapy for female substance users

Reyre, A., Jeannin, R., Largueuche, M., Moro, M, R., Barbet, T., Olivier, T. (2017). Overcoming professionals challenging experiences to promote a trustful therapeutic alliance in addiction treatment: A qualitative study. Drug and Alcohol Dependence. Volume 174. P.30-38.

Rhodes, T., Stimson, G., Moore, D., Bourgois, P., (2010). Qualitative social research in addictions publishing: Creating an enabling environment. International Journal of Drug Policy. Nov; 21(6): 441-444

Rizq, R., \& Target, M. (2009). The power of being seen: an interpretative phenomenological analysis of how experienced counselling psychologists describe the meaning and significance of personal therapy in clinical practice. Counselling Psychology Review, 24(3\&4): 66-83.

Rizq, R. (2007). On the margins: A psychoanalytic perspective on the location of counselling, psychotherapy and counselling psychology training programmes within universities. British Journal of Guidance and Counselling. 283-297.

Robbins, S., \& Jolkovski, M. (1987). Managing countertransference feelings: an interactional model using awareness of feeling and theoretical framework. Journal of Counseling Psychology, 34(3): 276-282.

Rodriguez, L., \& Smith, J.A. (2014). 'Finding your own place': An Interpretative Phenomenological Analysis of Young Men's Experience Of Early Recovery from Addiction. International Journal of Mental Health and Addiction 12: 477. doi:10.1007/s11469-014-9479-0

Rogers, C. (1961). On Becoming a Person. London: Constable \& Robinson.

Rouse, L. (2012). Evaluating qualitative research: Are we judging by the wrong standards? JEPS Bulletin. Blog.efpsa.org

Sack, D. (2012). Why relapse isn't a sign of failure. Psychology Today. Retrieved January 16, 2016 from https://www.psychologytoday.com/blog/where-science-meetsthe-steps/201210/why-relapse-isnt-sign-failure 
Helpful and unhelpful processes in psychological therapy for female substance users

Satre, J.P. (1958). Being and Nothingness. New York. Philosophical Library.

Shaffer, H.J. (1986). Conceptual crises and the addictions. A philosophy of science perspective. Journal of Substance Abuse Treatment. Vol.3 pp.285-296

Shinebourne, P., \& Smith, J, A. (2009). Alcohol and the self: an interpretative phenomenological analysis of the experience of addiction and its impact on the sense of self and identity. Addiction Research \& Theory 17 (2), pp. 152-167. ISSN 16066359.

Shinebourne, P., \& Smith, J, A. (2010). The communicative power of metaphors: an analysis and interpretation of metaphors in accounts of the experience of addiction. Psychology and Psychotherapy: Theory, Research and Practice. 83. 59-73. Shinebourne, P., \& Smith, J. A. (2011). 'It is just habitual': an interpretative phenomenological analysis of the experience of long-term recovery from addiction. International Journal of Mental Health and Addiction, 9, 282-295.

Simpson, D, D. (2004). A conceptual framework for drug treatment process and outcomes. Journal of Substance Abuse Treatment, 27, 99-121.

Smith, J.A. (2007). 'Hermeneutics, human sciences and health: linking theory and practice'. International Journal of Qualitative Studies on Health and Wellbeing. 2:311.

Smith, J. A., Flowers, P., \& Larkin, M. (2009). Interpretative phenomenological analysis: Theory, method and research. London; Los Angeles: SAGE.

Smith, J. A., \& Osborn, M. (2003). Interpretative phenomenological analysis. In J.A. Smith (ed.), Qualitative Psychology, A Practical Guide To Research Methods. 51-80. London: SAGE.

Spagnolo, P. A., Colloca, L., \& Heilig, M. (2015). The Role of Expectation in the Therapeutic Outcomes of Alcohol and Drug Addiction Treatments. Alcohol and Alcoholism (Oxford, Oxfordshire), 50(3), 282-285. 
Helpful and unhelpful processes in psychological therapy for female substance users

http://doi.org/10.1093/alcalc/agv015

Spinelli, E. (1989). The Interpreted World. An Introduction To Phenomenological Psychology. Sage. London.

Substance Abuse and Mental Health Services Administration (SAMHSA) (2014).

Retrieved February 10, 2016 from

http://www.samhsa.gov/data/sites/default/files/NSDUH-DR-FRR3-2014/NSDUH-

DR-FRR3-2014/NSDUH-DR-FRR3-2014.htm.

Substance Abuse and Mental Health Services Administration (SAMHSA), Office of Applied Studies. National Survey of Substance Abuse Treatment Services (NSSATS): (2010). Rockville, MD: DHHS Publication No. (SMA) 07-4296.

Taite, R. (2014). Five important advances in addiction treatment. The Science Of Addiction. Retrieved January 11, 2017 from https://blogs.psychcentral.com/scienceaddiction/2014/06/5-important-advances-in-addiction-treatment/

Taite, R. (2014). Addiction: Identity and Connection Influence Relapse Rates. Psychology Today. Retrieved June 2016, from https://www.psychologytoday.com/blog/ending-addiction-good/201403/addictionidentity-and-connection-influence-relapse-rates

The National Institute for Health and Care Excellence, NICE (2016). Drug misuse prevention overview. Retrieved March, 2016, from https://pathways.nice.org.uk/pathways/drug-misuse-prevention

The National Centre on Addiction and Substance Abuse (CASA) at Columbia University.(2012). Addiction Medicine: Closing the gap between science and practice. Retrieved October 12, 2016, from http://www.centeronaddiction.org/addiction$\underline{\text { research/reports/addiction-medicine-closing-gap-between-science-and-practice }}$

Timulak, L. (2007). Identifying core categories of client-identified impact of helpful events in psychotherapy: A qualitative meta-analysis. Psychotherapy Research, 17(3), 310-320. 
Helpful and unhelpful processes in psychological therapy for female substance users

Tollin, D. F. (2010). Is cognitive-behavioural therapy more effective than other therapies? A meta-analytic review. Cognitive Behaviour Therapy, Vol. 44, No. 2, 162-174, http://dx.doi.org/10.1080/16506073.2014.998705

Tuchman, E. (2010). Women and Addiction: The importance of gender issues in substance abuse research. Journal of Addictive Diseases. 29:2, 127-138.

UKATT Research Team. (2005). Effectiveness of treatment for alcohol problems: findings of the randomised United Kingdom Treatment Trial. British Medical Journal, 331, 541-544.

Unnithan S., Gossop M., \& Strang, J. (1992). Factors associated with relapse among opiate addicts in an outpatient detoxification programme. British Journal of Psychiatry, 161, 654-657.

UNODC (2016). World Drug Report. Retrieved October 16, 2017 from http://www.unodc.org/doc/wdr2016/WORLD_DRUG_REPORT_2016_web.pdf

Vaillant, G. E. (1983). The natural history of alcoholism: Causes, patterns, and paths to recovery. Cambridge: Harvard University Press.

Vaillant, G. (2005). Alcoholics Anonymous: cult or cure? Australian and New Zealand Journal of Psychiatry, 39, 431-436.

Vale, P. H. (2009). Addiction and rational choice theory. International Journal of Consumer Studies, 34: 38-45. doi:10.1111/j.1470-6431.2009.00826.x

Wachtel, P.L. (2007) Relational Theory and the Practice of Psychotherapy, New York: The Guildford Press

Walitzer, K., \& Dearing, R. L. (2006). Gender differences in alcohol and substance use relapse. Clinical Psychology Review. 26: 128-148

Warnock, M. (1987). Memory. London. Faber and Faber 
Helpful and unhelpful processes in psychological therapy for female substance users

Waters, K., Holttum., Perrin, I. (2014). Narrative and attachment in the process of recovery from substance misuse. Psychology and Psychotherapy: Theory, Research and Practice. 87, 222-236.

Watson, L., \& Parke, A. (2011) 'Experience of Recovery for Female Heroin Addicts: An Interpretative Phenomenological Analysis'. International Journal of Mental Health Addiction 9, p102-117.

Weegman, M., \& Cohen, R. (2002). The Psychodynamics of Addiction. Whurr Publishers: London.

West, R. (2006) Theory of Addiction. Oxford: Blackwell.

Willig, C. (2007). Reflections on the use of a phenomenological method. Qualitative Research in Psychology, (4), 209-225. doi:10.1080/ 14780880701473425

Willig, C. (2011). The ethics of interpretation. 255-272. Inaugural Professorial Lecture. Willig, C. (2013). Introducing qualitative research in psychology (3rd ed.). Maidenhead: McGraw-Hill Education.

Willig, C. (2008). Introducing qualitative research in psychology: Adventures in theory and method. Maidenhead: McGraw-Hill Open University Press.

Willig, C, \& Stainton-Rogers, W. (2013). The SAGE Handbook of Qualitative Research In Psychology. Sage Publications.

Whitmore, R., Chase, S. K., Mandle, L, C. (2001) Validity in qualitative research. Qualitative Health Research. Vol 11. No.4. 522-537. Sage Publications

Woolfe, R., Dryden, W., Strawbridge, S. (2003) Handbook of Counselling Psychology. London: Sage.

Worley, J. (2017). Recovery in substance use disorders: what to know to inform practice. Issues in Mental Health Nursing. Volume. 38. Issue 1.

World Health Organisation, (WHO). (2010). World Strategy to reduce the harmful use of alcohol. Retrieved July 2016. From: 
Helpful and unhelpful processes in psychological therapy for female substance users

http://www.who.int/substance_abuse/msbalcstragegy.pdf

Yardley, L. (2000). Dilemmas in qualitative health research. Psychology and Health, 15, 215- 228. doi:10.1080/ 08870440008400302

Yardley, L. (2011). Demonstrating validity in qualitative research. In J. A. Smith (Ed.), Qualitative psychology: A practical guide to research methods (pp. 234251). London: SAGE.

Yin, R. K. (2011). Qualitative research from start to finish. New York: Guilford Press.

Zur, O. (2016). Self-Disclosure \& Transparency in Psychotherapy and Counselling: To Disclose or Not to Disclose, This is the Question. Retrieved January 18, 2017 from http://www.zurinstitute.com/selfdisclosure1.html. 


\title{
IIIII University of Roehampton
}

\author{
London
}

Example of an email sent to Charity Organisations that will be contacted for

\section{recruiting participants for the study (Appendix 1)}

To Whom It May Concern:

I am interested in undertaking research into the experience of women that have undertaken psychological therapy for substance use disorder. I will be focusing on exploring what factors they found helpful and unhelpful in this process. I am looking to recruit women for this study who would be willing to take part in a confidential interview exploring their experience of psychological therapy. The interviews will be digitally recorded and analysed via IPA, a qualitative methodology.

The study has been approved by the University research board and subsequently received Ethical approval from the committee. I wonder if you would be able to help me with recruiting participants for this study? I am aware your organisation seeks to support and advise women in this situation, and it would be most helpful if, for example, there were an opportunity for me to meet some of the women that have recently completed therapy, and introduce myself and the aims of the study. Would it be possible for you to write to former clients to see whether they would be willing to participate. Any participants would be greatly appreciated.

The study aims primarily to explore what this client group found helpful or not in their one to one counselling. This is with a view to increasing the research that is available in this area and the likelihood that women will receive the help they need.

I would be most grateful of you could let me know whether this might be possible. I would be pleased to discuss this further with you - I can be contacted on 07515775720 . Alternatively, I would be happy to telephone you at a time suitable for you.

I look forward to hearing from you.

Kind regards

Jane Halsall (Trainee Counselling Psychologist) - halsallj@roehampton.ac.uk

Mick Cooper - Tel $02083923741 \quad$ Dr Diane Bray - Tel 0208392327

Email-mick.cooper@roehampton.ac.ukEmail - D.Bray@roehampton.ac.uk

(Principal Lecturer and Supervisor) (Head of academic department) 


\section{University of}

London

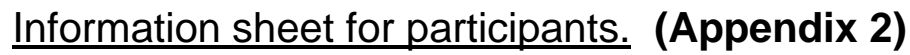

- Psychological therapy for substance use disorder from a woman's perspective. An Interpretive Phenomenological Analysis of helpful and unhelpful factors.

I am a student and trainee with the University of Roehampton undertaking a Doctoral programme in Counselling Psychology. The study is being carried out as part of the programme.

The study aims to explore what women abusing drugs or alcohol (diagnosed with substance use disorder) found helpful or unhelpful in psychological therapy. With the rise of women seeking treatment for drug and alcohol addiction it is important that their voices get heard in the literature. This research would enable women to share their experience of therapy; be able to self reflect and contribute to the research. In identifying factors, it would inform practitioners how to tailor treatment plans and enable them to work more effectively for this client group. It is noted that this topic may trigger some memories but you will be able to stop at any point if you feel any distress.

Participants who agree to take part in this study will be asked about their psychological therapy, specifically the helpful and non-helpful aspects. The interview will be digitally recorded so that I can write up and explore what is said to me during the interview at a later date. The tapes will be kept securely for up to ten years after which they will be destroyed. All identifying information will be removed from the transcripts to protect participant anonymity and maintain confidentiality. The interview should take approximately 1 - 2 hours and you will have an opportunity to read your transcript once it has been completed.

Participants agreeing to take part in this study will be treated confidentially and this means that everything that is said to me during the interview will be treated as private and will not be shared. However, if there is a disclosure of self harm or risk to others then there would be an ethical obligation to share this information with my supervisor. This would be discussed in depth, before any action was taken.

Details of various organisations whom you can contact for help and advice should you need to speak to someone or if you are upset and distressed 
following anything that arises from the interview today are listed below. Those contacts are outlined in this form and on the participant de-brief that will be supplied to you at the end of the interview.

If you have any questions regarding the interview, the aims of that and its overall purpose then please do not hesitate to ask me or the supervisory team, Mick Cooper or Diane Bray.

Thank you

Jane Halsall (Trainee Counselling Psychologist)

\section{Useful contact numbers for support}

Action on Addiction - HOPE HOUSE - 03003300659

Addaction - 02086779541

Mind - 03301233393

Womens Work Limited - 01332227741

\section{Contact}

Department of Psychology University of Roehampton, Whitelands College Holybourne Avenue SW15 4JD

Telephone - 02083923000

Researcher - Jane Halsall

(halsallj@roehampton.ac.uk)

Mick Cooper- Tel $02083923741 \quad$ Dr Diane Bray - Tel 0208392327

Email:mick.cooper@roehampton.ac.uk Email-D.Bray@roehampton.ac.uk (Principal Lecturer and Supervisor) (Head of academic department) 


\title{
IIIIIIUniversity of Roehampton
}

(Appendix 3)

\author{
London
}

\section{PARTICIPANT CONSENT FORM}

Title of Research Project: Psychological therapy for substance use disorder from a woman's perspective. An Interpretive Phenomenological Analysis of helpful and unhelpful factors.

\section{Brief Description of Research Project, and What Participation Involves:}

Thank you for your interest in participating in this study. I am hoping to explore what women with a diagnosis of substance use disorder found helpful or unhelpful in their psychological therapy. This research aims to explore the factors that women themselves identified, in order to give a voice to this client group. Understanding the experiences of women's psychological therapy is an important area of research as the number of woman being diagnosed with substance use disorder is on the increase. In identifying helpful and unhelpful aspects, then treatment could be tailored to enable them to get the help they might need. I am hoping to recruit approximately $8-10$ female participants and the interviews will take approximately 1 - 2 hours in a mutually agreed location.

\section{Investigator Contact Details:}

Name - JANE HALSALL

Department - Psychology

University address -

University of Roehampton,

Whitelands College,

Holybourne Avenue,

Postcode - SW15 4JD

Email - halsallj@roehampton.ac.uk

Telephone - 02083923000

\section{Consent Statement:}

I agree to take part in this research, and am aware that I am free to withdraw at any point without giving a reason, although if I do so I understand that my data might still be used in a collated form. I understand that the information I provide will be treated in confidence by the investigator and that my identity will be protected in the publication of any findings, and that data will be collected and processed in accordance with the Data Protection Act 1998 and with the University's Data Protection Policy. 
Helpful and unhelpful processes in psychological therapy for female substance users

Name

Signature

Date

\section{Withdrawal from study}

I understand that my participation is voluntary, that I can choose not to participate in part or all of the project, and that I can withdraw from the study at any point before my interview or up until one month after my interview is conducted. Withdrawal from the project will not result in being penalised or disadvantaged in any way.

Name: .(please print) Signature:

\section{Date:}

Researcher's signature:

Please note: if you have a concern about any aspect of your participation or any other queries please raise this with the investigator (or if the researcher is a student you can also contact the Director of Studies.) However, if you would like to contact an independent party please contact the Head of Department.

\section{Director of Studies Contact Details: Head of Department Contact Details:}

Name - Mick Cooper

University Address -

University of Roehampton,

Whitelands College,

Holybourne Avenue,

SW15 4JD

Email -mick.cooper@roehampton.ac.ukEmail - D.Bray@roehampton.ac.uk Telephone - 02083923741
Name - Dr Diane Bray

University of Roehampton

Whitelands College

Holybourne Avenue

SW15 4JD

\section{Telephone - 02083923627}




\section{IIIIXY University of Roehampton \\ London}

\section{Title of Research Project:}

Psychological therapy for substance use disorder from a woman's perspective. An Interpretive Phenomenological Analysis of helpful and unhelpful factors.

\section{Demographic Questionnaire (Appendix 4)}

Please can you answer the following questions;

Age

Ethnicity

Length of time in psychological therapy....

Length of time since completing treatment

Drug that you were addicted to.

Residential or Daycare Program.

Thank you

JANE HALSALL (Trainee Counselling Psychologist) 


\section{IIIIIIUniversity of Roehampton}

London

The interview schedule (Appendix 5)

Title of Research Project:

\section{Psychological therapy for substance use disorder from a woman's}

perspective. An Interpretive Phenomenological Analysis of helpful and unhelpful factors.

The following questions will be used to guide the interview

1) What was helpful in terms of what the counsellor did (i.e. interventions / relationship) specifically for your substance use disorder?

2) What was most helpful in terms of what you did as the client?

3) Why was it helpful?

4) What was unhelpful in terms of what the counsellor did? (Lack of empathy, didn't listen)

5) What was most unhelpful in terms of what you did as the client specific to your disorder?

\section{Prompts;}

Can you tell me more about that?

What did that mean to you?

Thank you

\section{Contact}

Department of Psychology

University of Roehampton,

Whitelands College

Holybourne Avenue

SW15 4JD

Telephone - 02083923000 
Helpful and unhelpful processes in psychological therapy for female substance users

Researcher - JANE HALSALL (halsallj@roehampton.ac.uk )

Mick Cooper - Tel 02083923741 Dr Diane Bray - 02083923627

Email - Mick.cooper@roehampton.ac.ukEmail - D.Bray@roehampton.ac.uk (Principal Lecturer and Supervisor) (Head of academic department) 


\title{
University of Roehampton
}

\author{
London
}

\section{Participant debrief (Appendix 6)}

Title of Research Project:

\section{Psychological therapy for substance use disorder from a woman's}

perspective. An Interpretive Phenomenological Analysis of helpful and

unhelpful factors.

Thank you very much for participating in the study. I understand that some of what you discussed today might be difficult and I would therefore like to leave you with these contact numbers should you wish to speak to someone following this study.

Action on Addiction - HOPE HOUSE - 03003300659

Addaction - 02086779541

Mind - 03301233393

Womens Work Limited - 01332227741

Samaritans - 08457909090

NHS Direct - 08454647

If you have any concerns about the study and wish to contact someone at the University of Roehampton then please contact;

Department of Psychology

University of Roehampton,

Whitelands College

Holybourne Avenue

SW15 4JD

Telephone - 02083923000

Researcher - Jane Halsall (halsallj@roehampton.ac.uk)

Mick Cooper- Tel 02083923741 Dr Diane Bray - 02083923627

Email - mick.cooper@roehampton.ac.ukEmail - D.Bray@roehampton.ac.uk (Principal Lecturer and Supervisor) (Head of academic department) 
Helpful and unhelpful processes in psychological therapy for female substance users

\section{Thank you}

Jane Halsall (Trainee Counselling Psychologist) 
Helpful and unhelpful processes in psychological therapy for female substance users

\section{Appendix 7 - Ethical Approval Email Confirmation}

From: Jan Harrison

Sent: 25 March 2015 08:29

To: Jane Halsall (Research Student)

Cc: Lance Slade; Mick Cooper

Subject: Ethics Application Ref: PSYC 15/ 165

Dear Jane,

\section{Ethics Application}

Applicant: Jane Halsall

Title: $\quad$ Psychological therapy for female substance use disorder. An

Interpretive Phenomenological Analysis of helpful and unhelpful factors.

Reference: PSYC 15/ 165

Department: Psychology

I am pleased to confirm that the risk assessment for your project has been reviewed and approved by the Head of Health \& Safety. Under the procedures agreed by the University Ethics Committee I am pleased to advise you that your Department has confirmed that all conditions for approval of this project have now been met. We do not require anything further in relation to this application.

Please note that on a standalone page or appendix the following phrase should be included in your thesis:

The research for this project was submitted for ethics consideration under the reference PSYC 15/ 165 in the Department of Psychology and was approved under the procedures of the University of Roehampton's Ethics Committee on 25.03.15.

Please advise us if there are any changes to the research during the life of the project. Minor changes can be advised using the Minor Amendments Form on the Ethics Website, but substantial changes may require a new application to be submitted.

Many thanks,

Jan

Jan Harrison?Ethics Officer, Research Office, Department of Academic Enhancement? Roehampton | London | SW15 5PJ

jan.harrison@roehampton.ac.uk|www.roehampton.ac.uk

Tel: +44 (0) 2083925785

Follow us on Twitter | Find us on Facebook

Consider the environment. Please don't print this e-mail unless you really need to. 
Helpful and unhelpful processes in psychological therapy for female substance users

Appendix 8. An example of my analysis

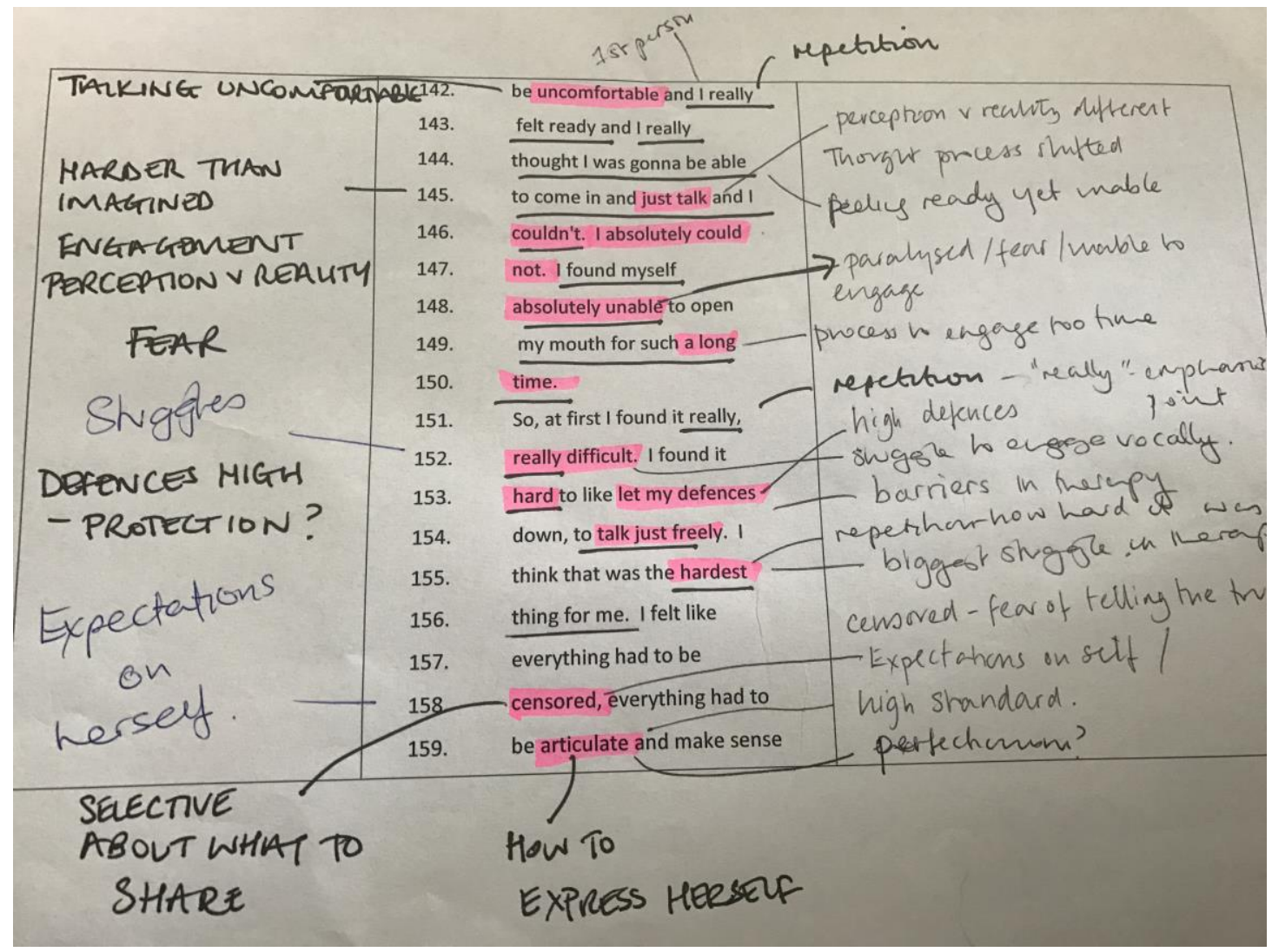


Helpful and unhelpful processes in psychological therapy for female substance users

Appendix 9: FULL TRANSCRIPT - PARTICIPANT SAM

\section{* (CERTAIN PAGES HAVE BEEN EXCLUDED THAT WOULD HAVE COMPROMISED HER IDENTITY).}
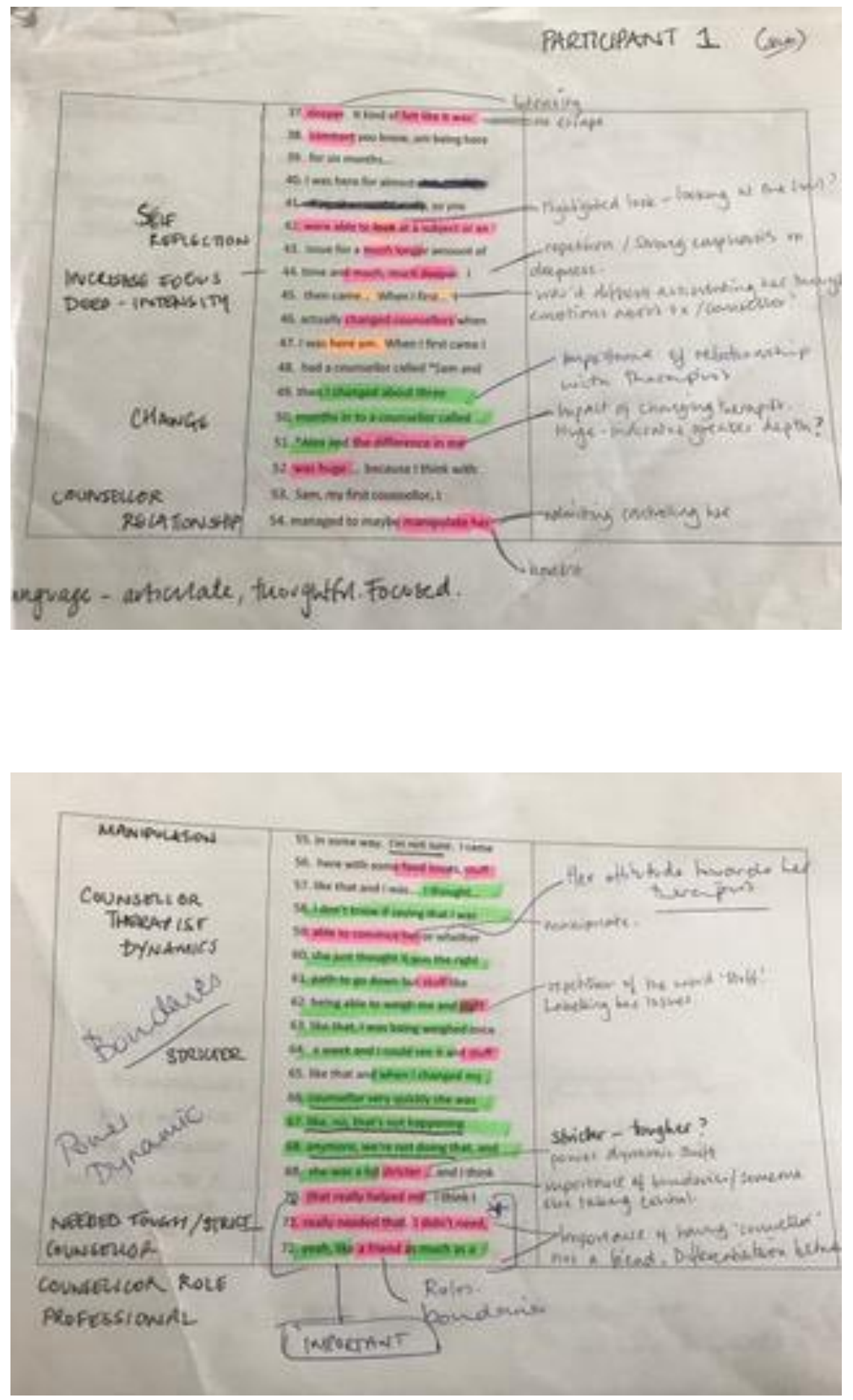
Helpful and unhelpful processes in psychological therapy for female substance users
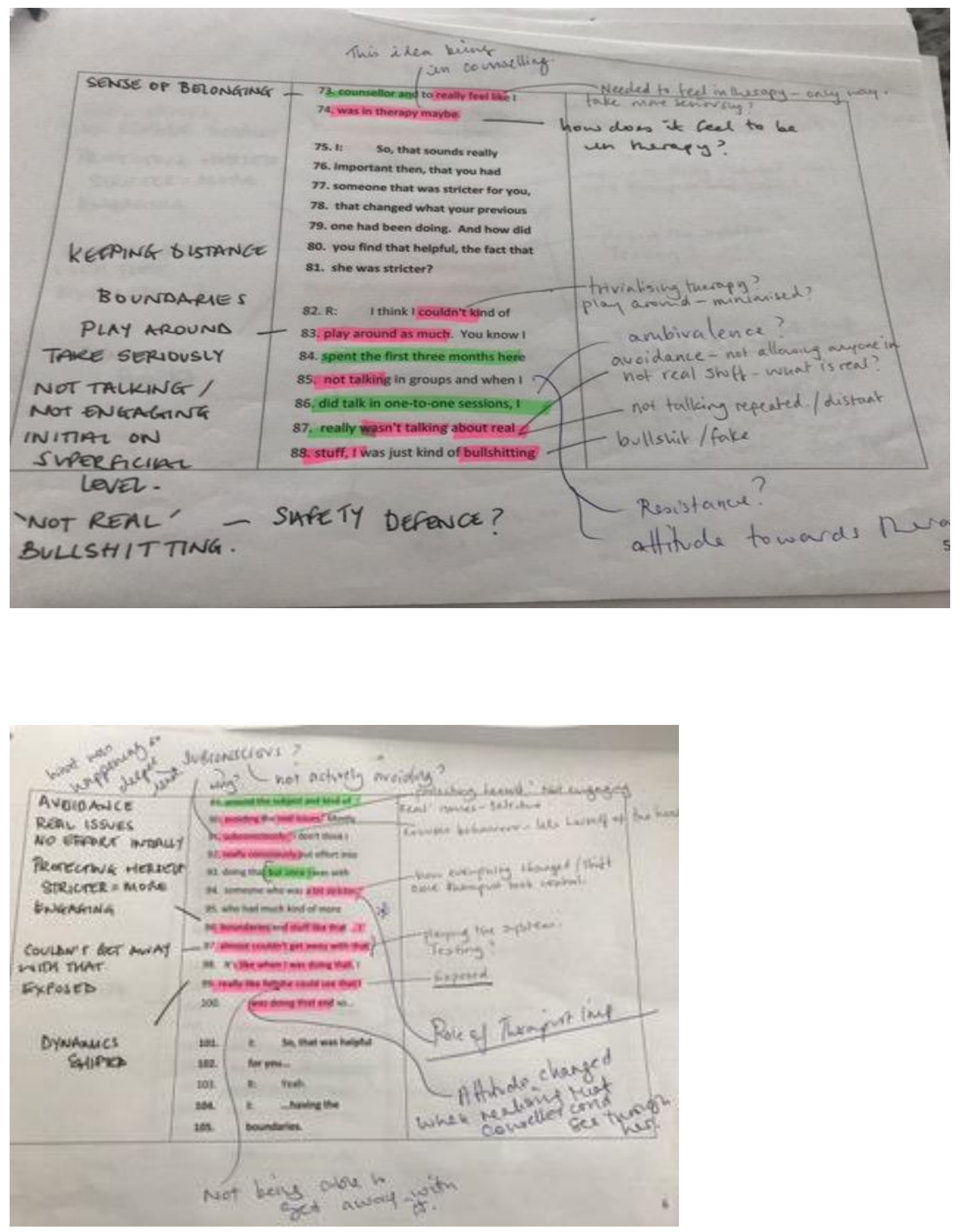
Helpful and unhelpful processes in psychological therapy for female substance users

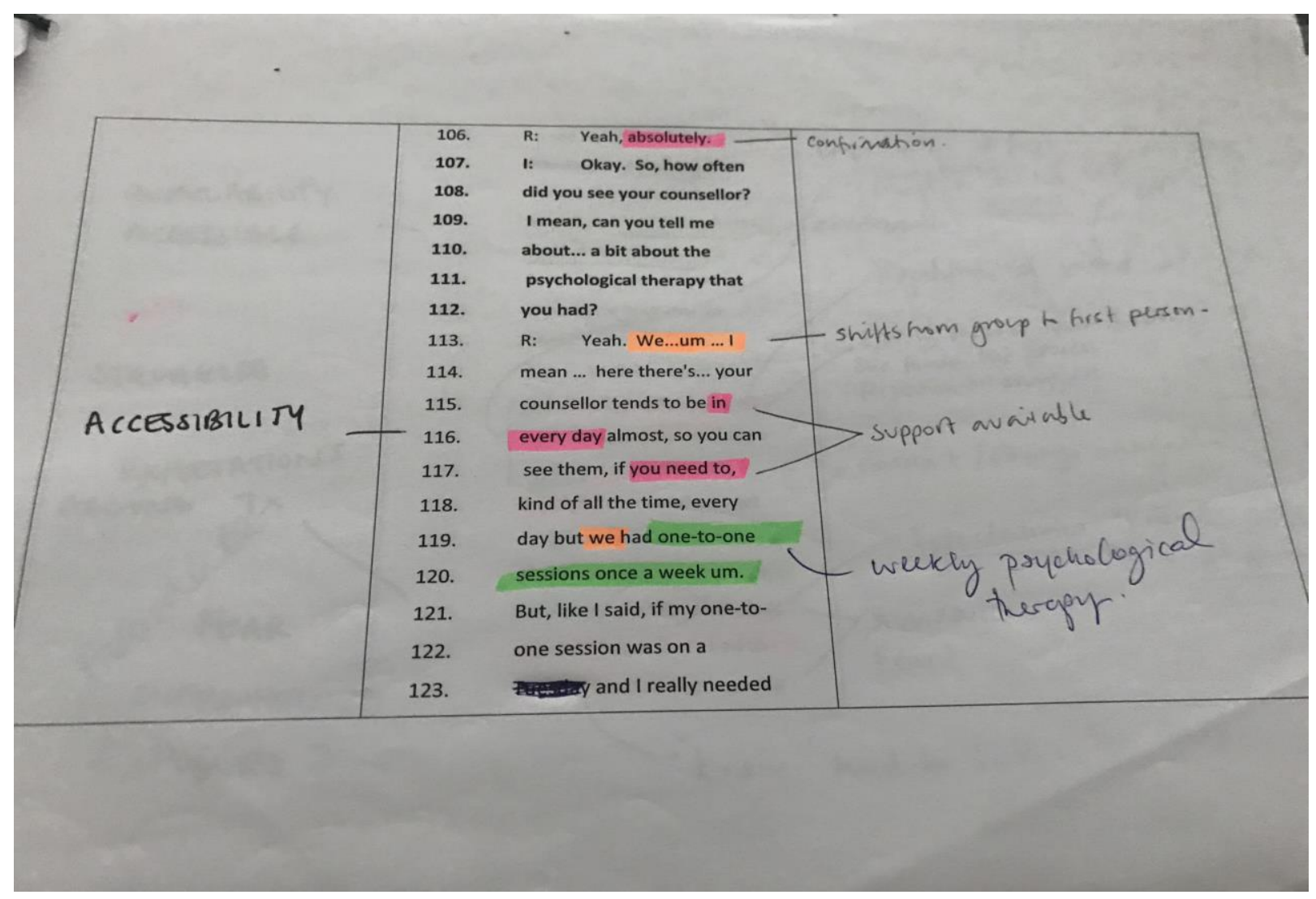


Helpful and unhelpful processes in psychological therapy for female substance users
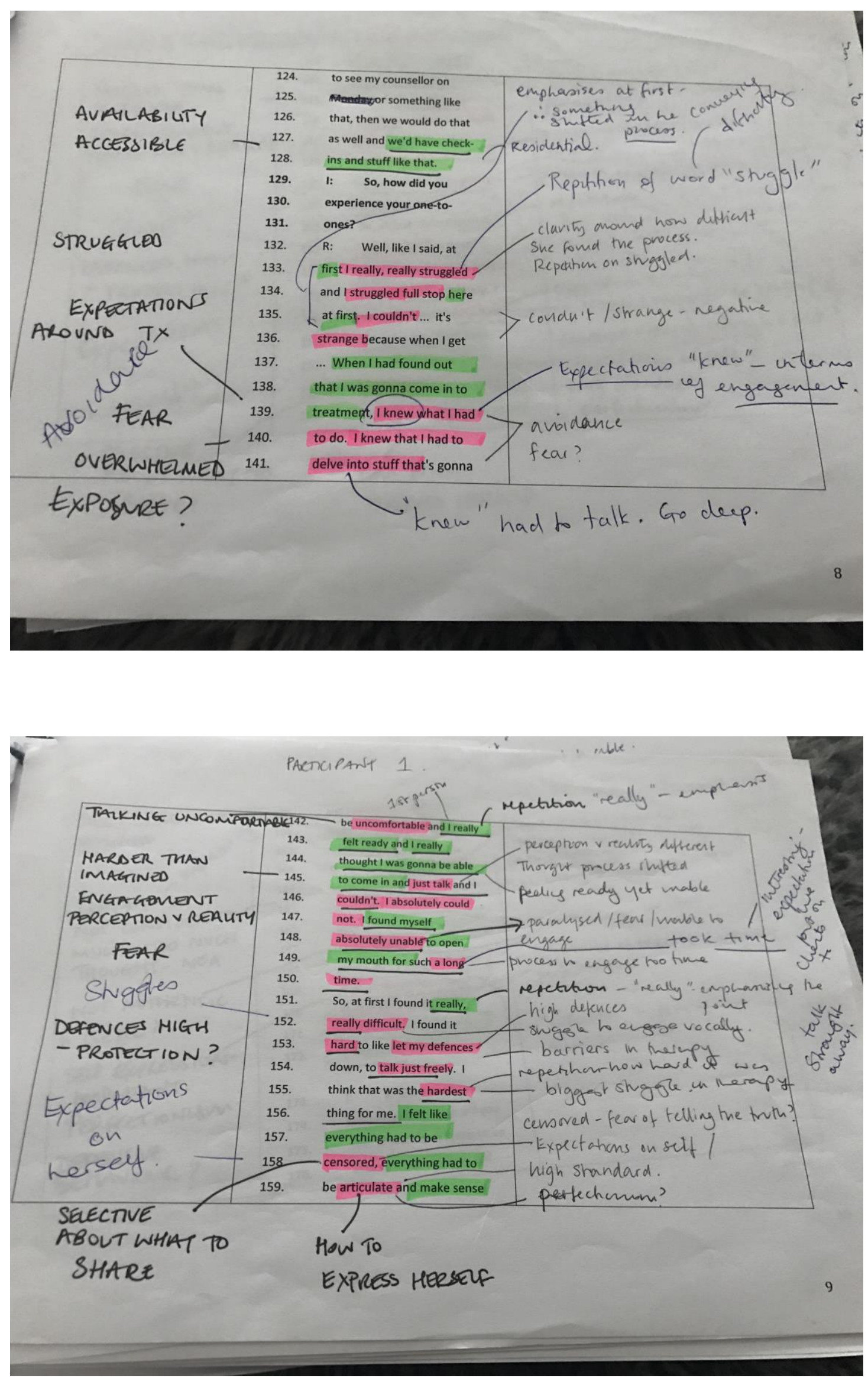
Helpful and unhelpful processes in psychological therapy for female substance users
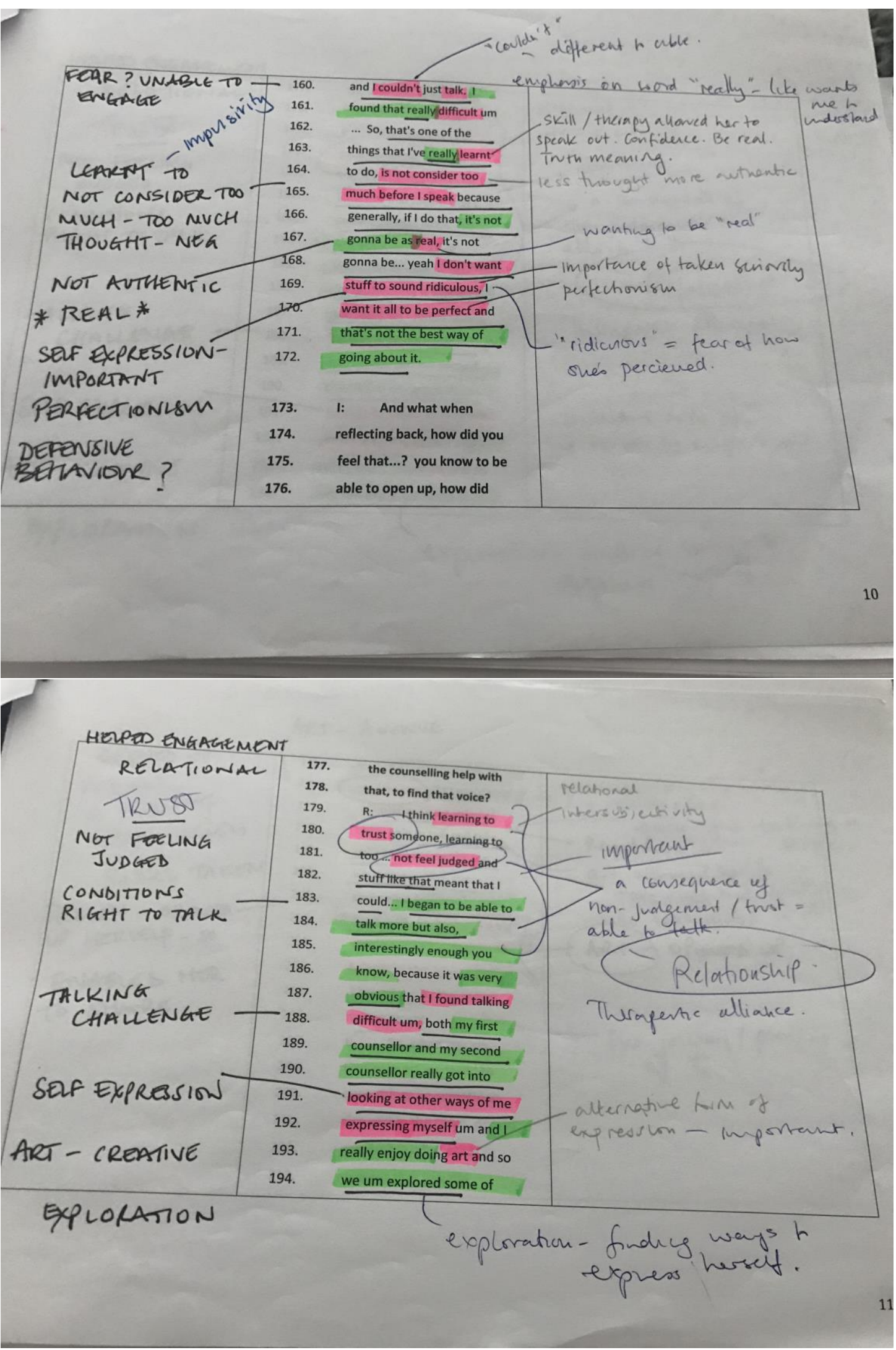
Helpful and unhelpful processes in psychological therapy for female substance users
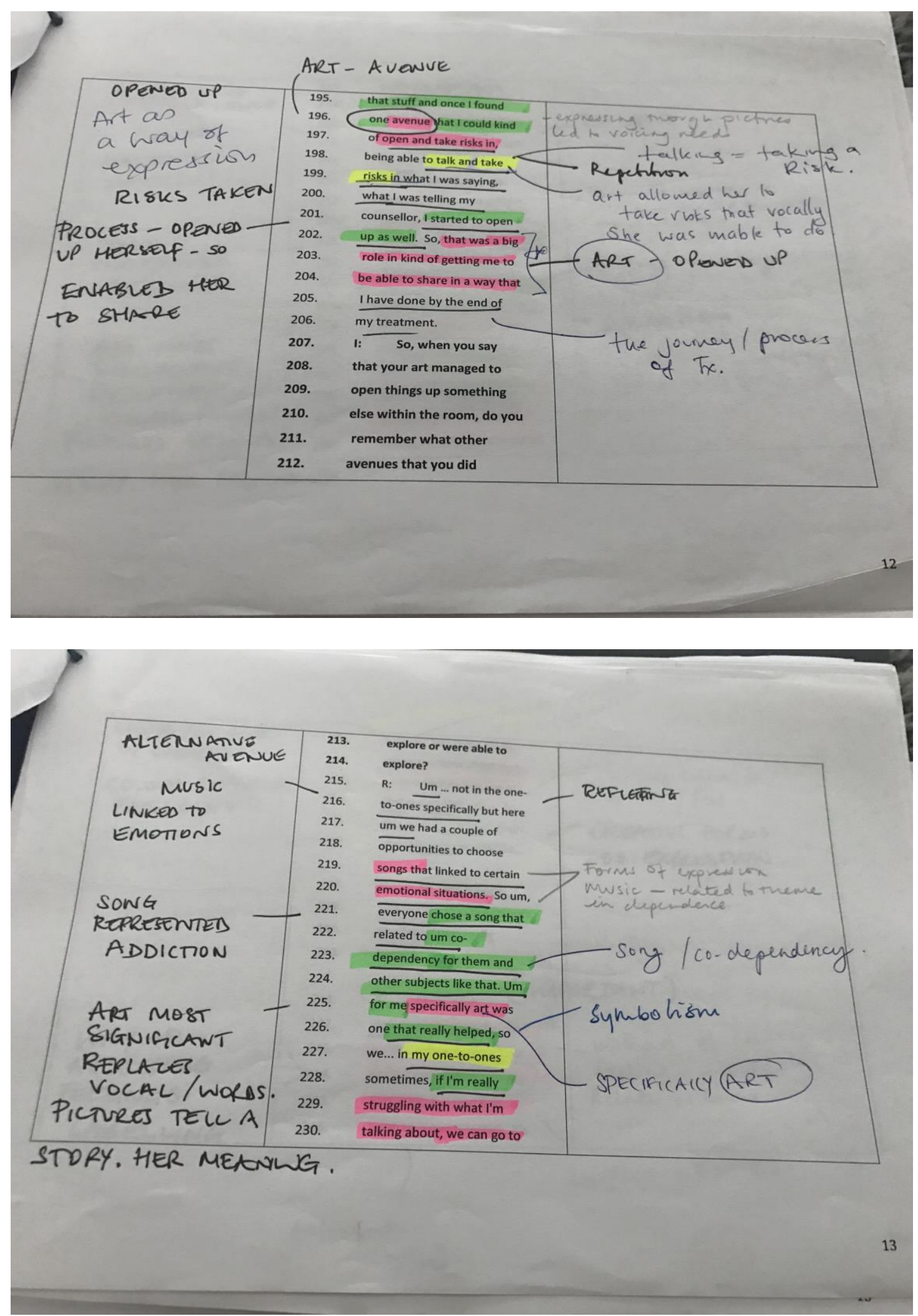
Helpful and unhelpful processes in psychological therapy for female substance users
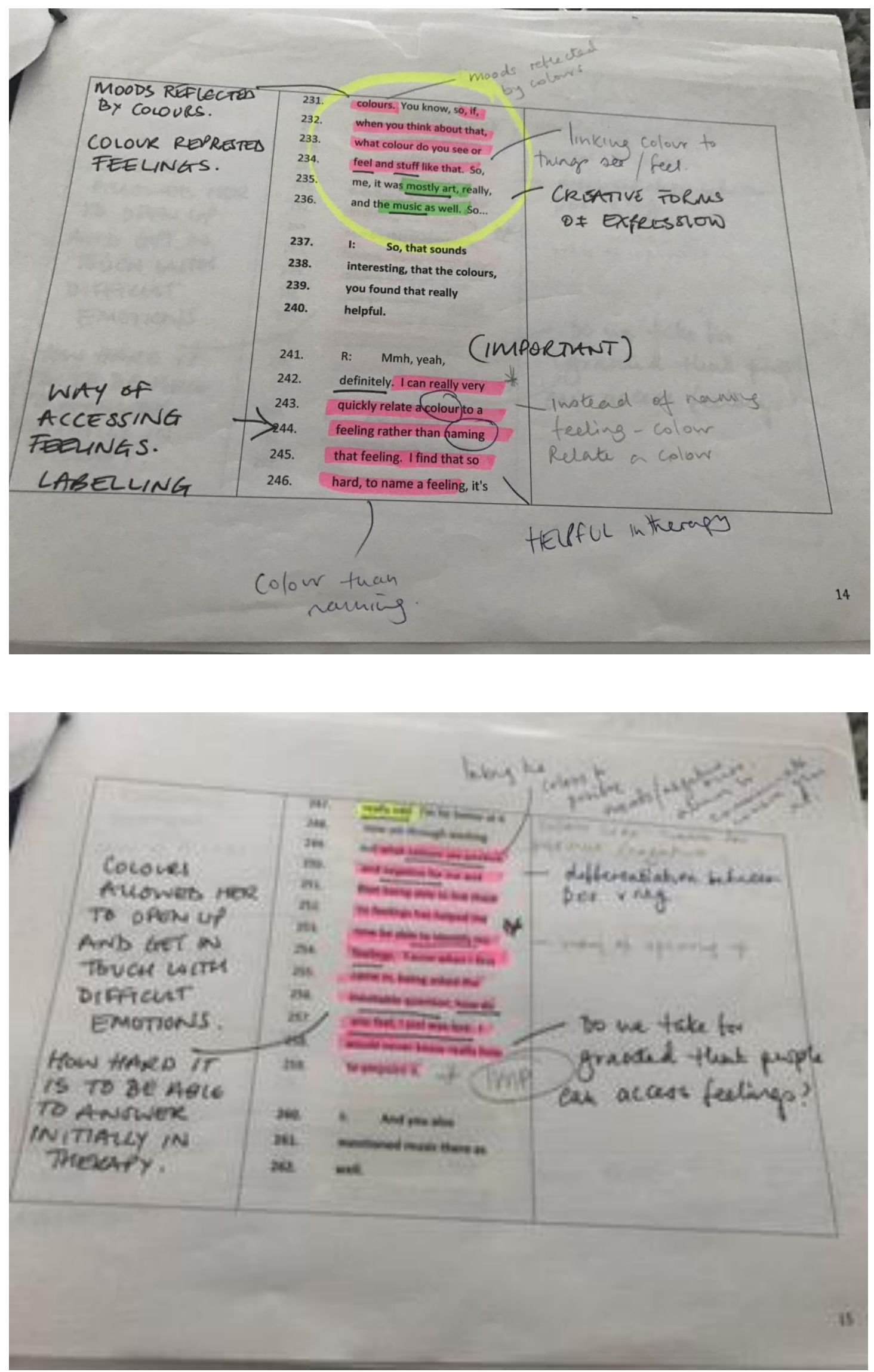
Helpful and unhelpful processes in psychological therapy for female substance users
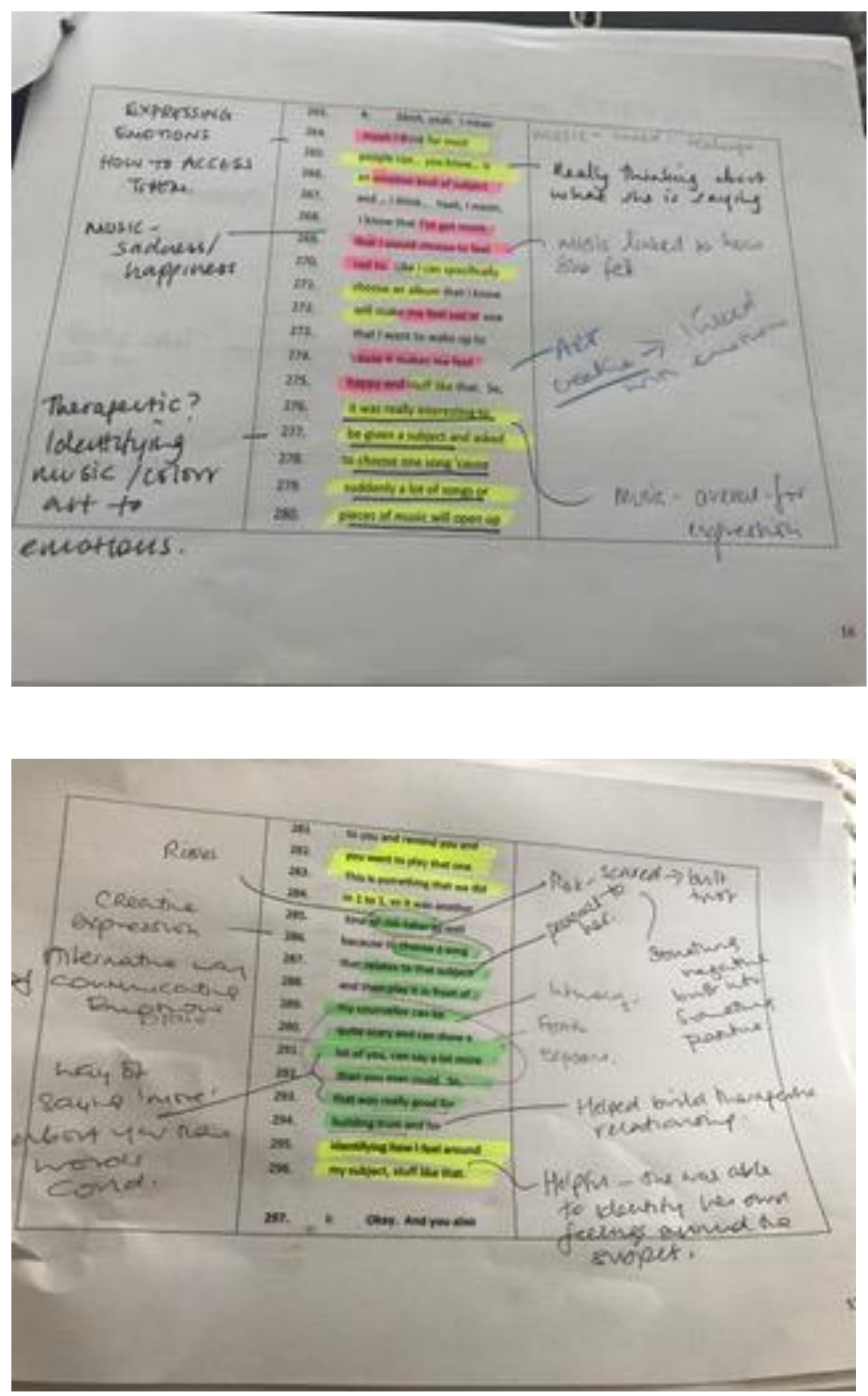
Helpful and unhelpful processes in psychological therapy for female substance users
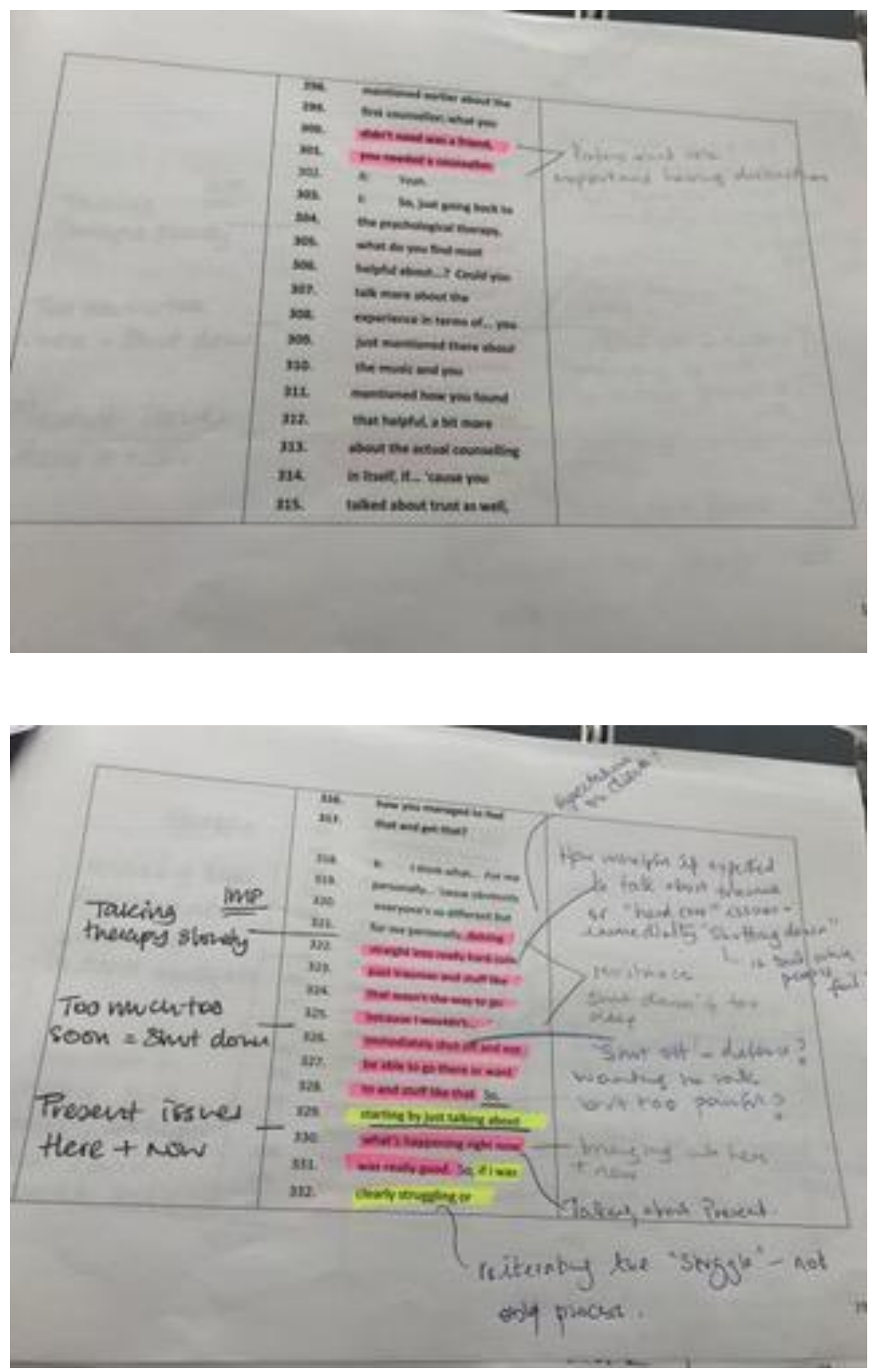
Helpful and unhelpful processes in psychological therapy for female substance users
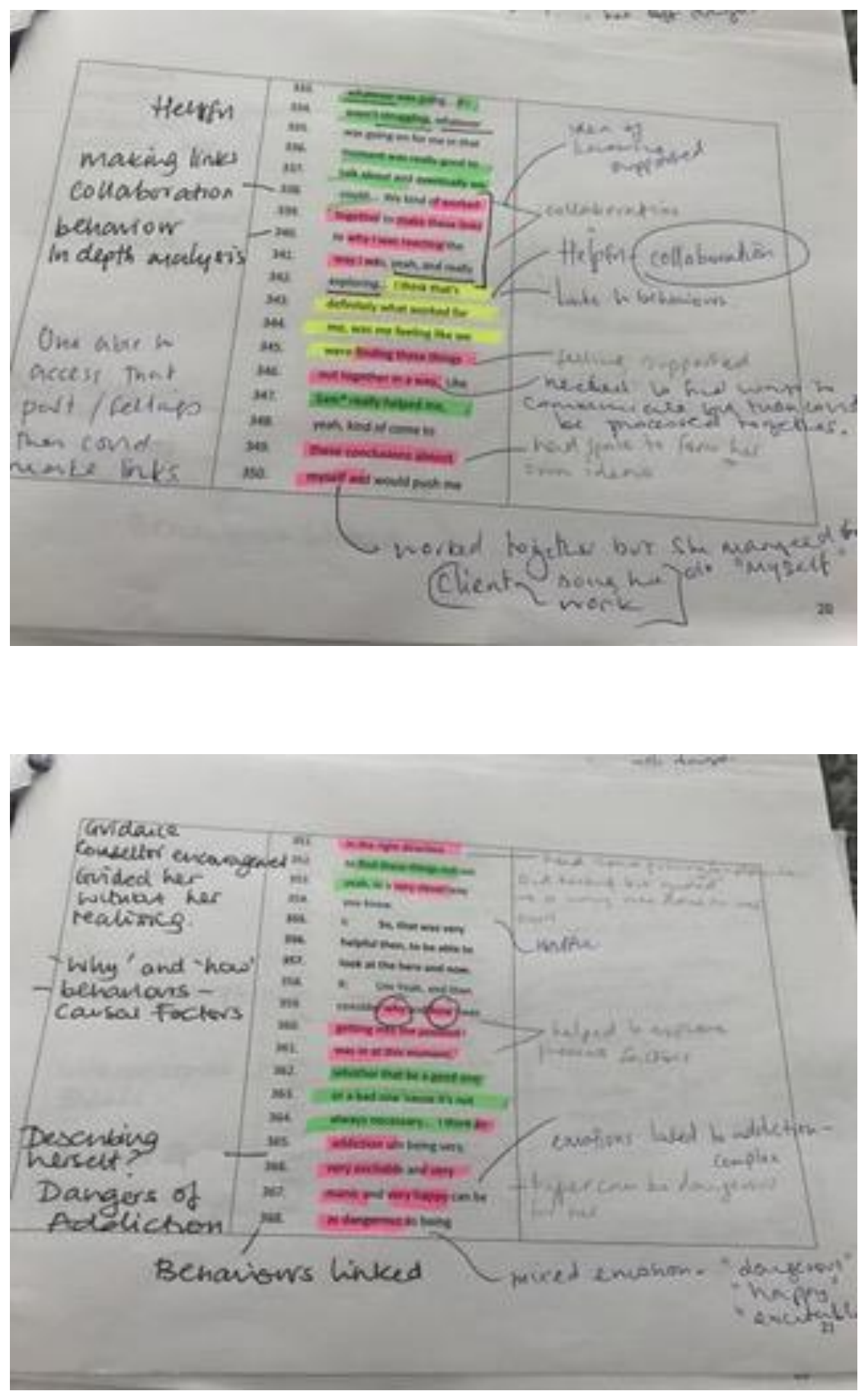
Helpful and unhelpful processes in psychological therapy for female substance users
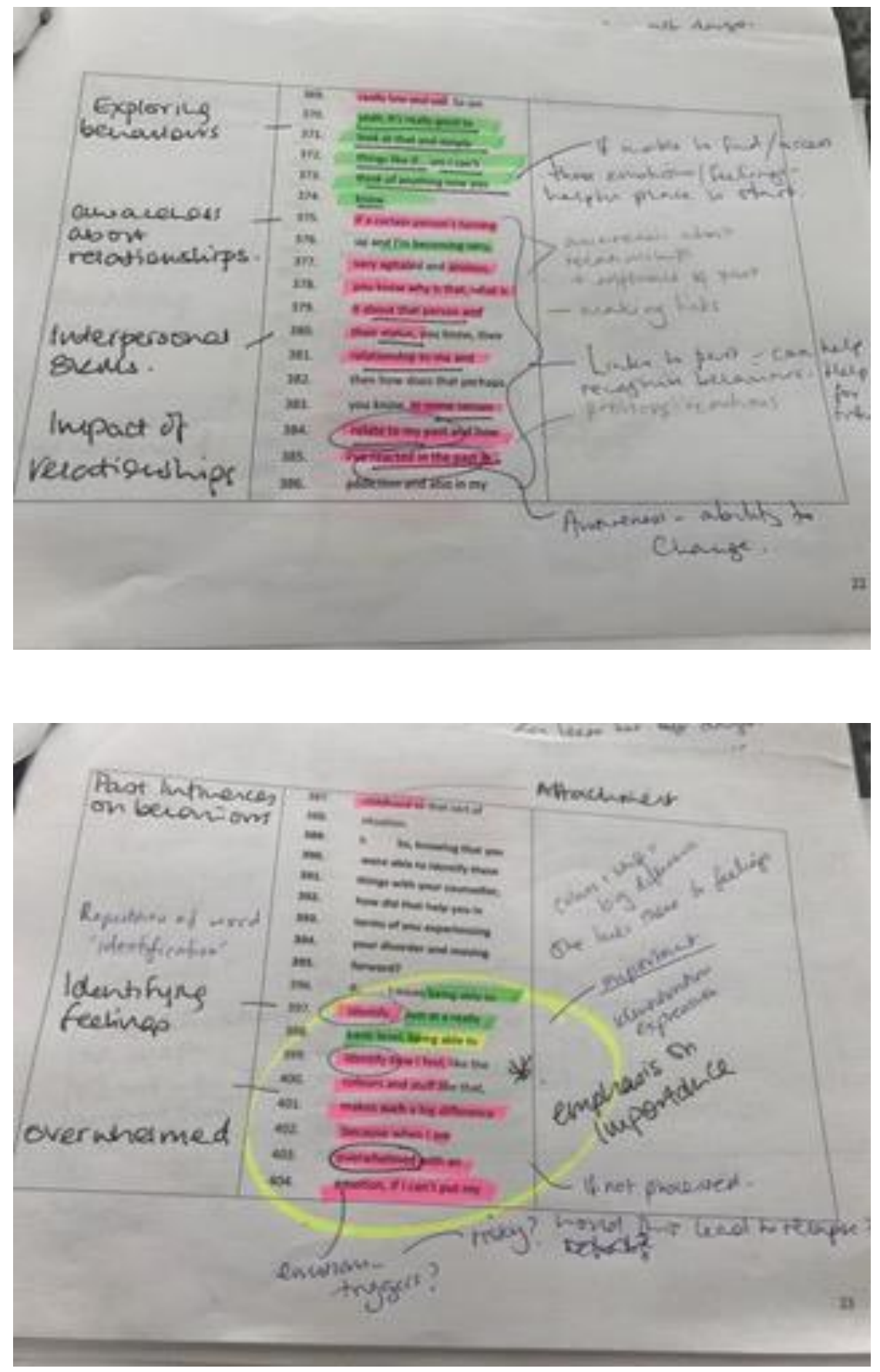
Helpful and unhelpful processes in psychological therapy for female substance users
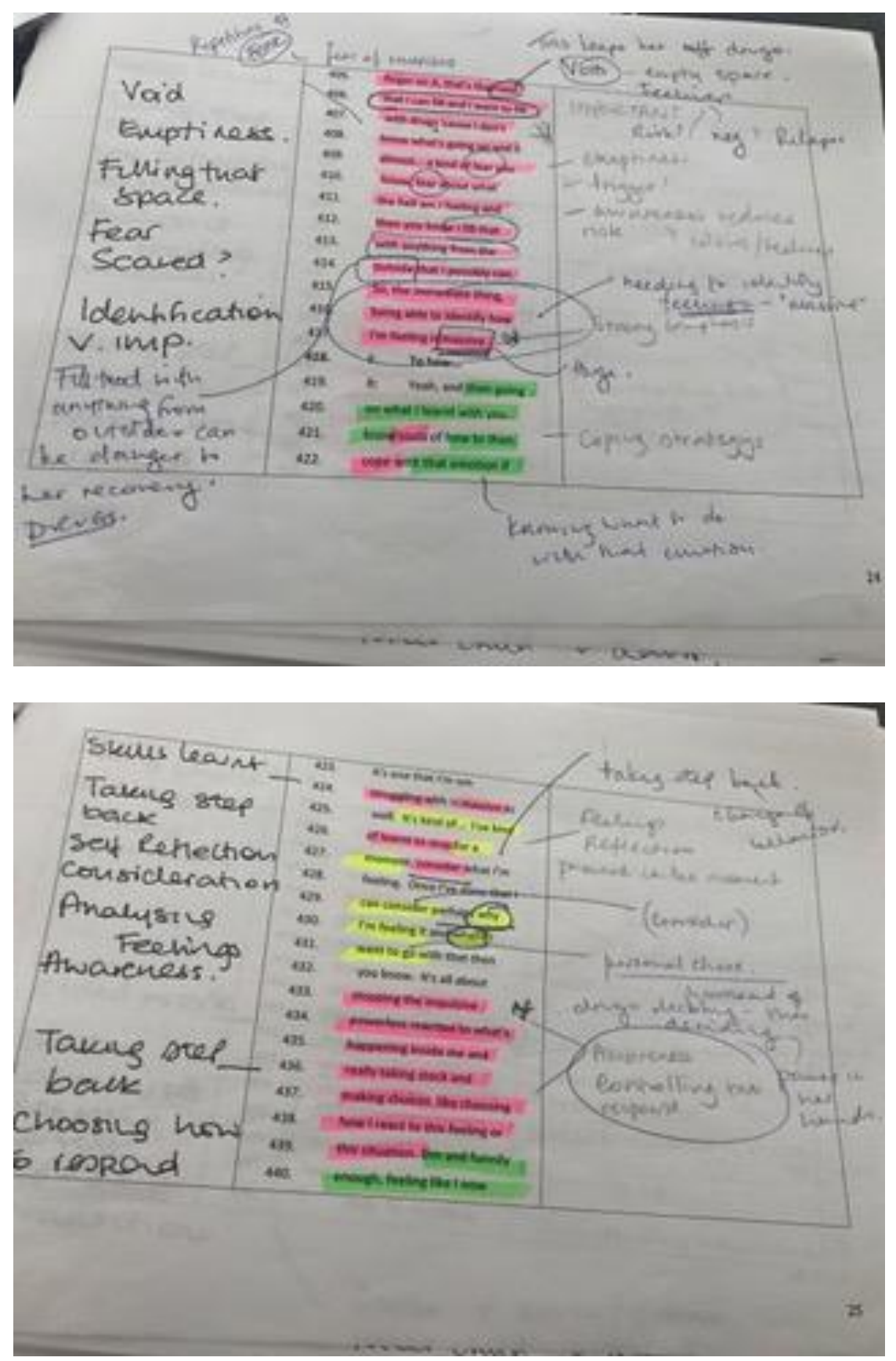
Helpful and unhelpful processes in psychological therapy for female substance users
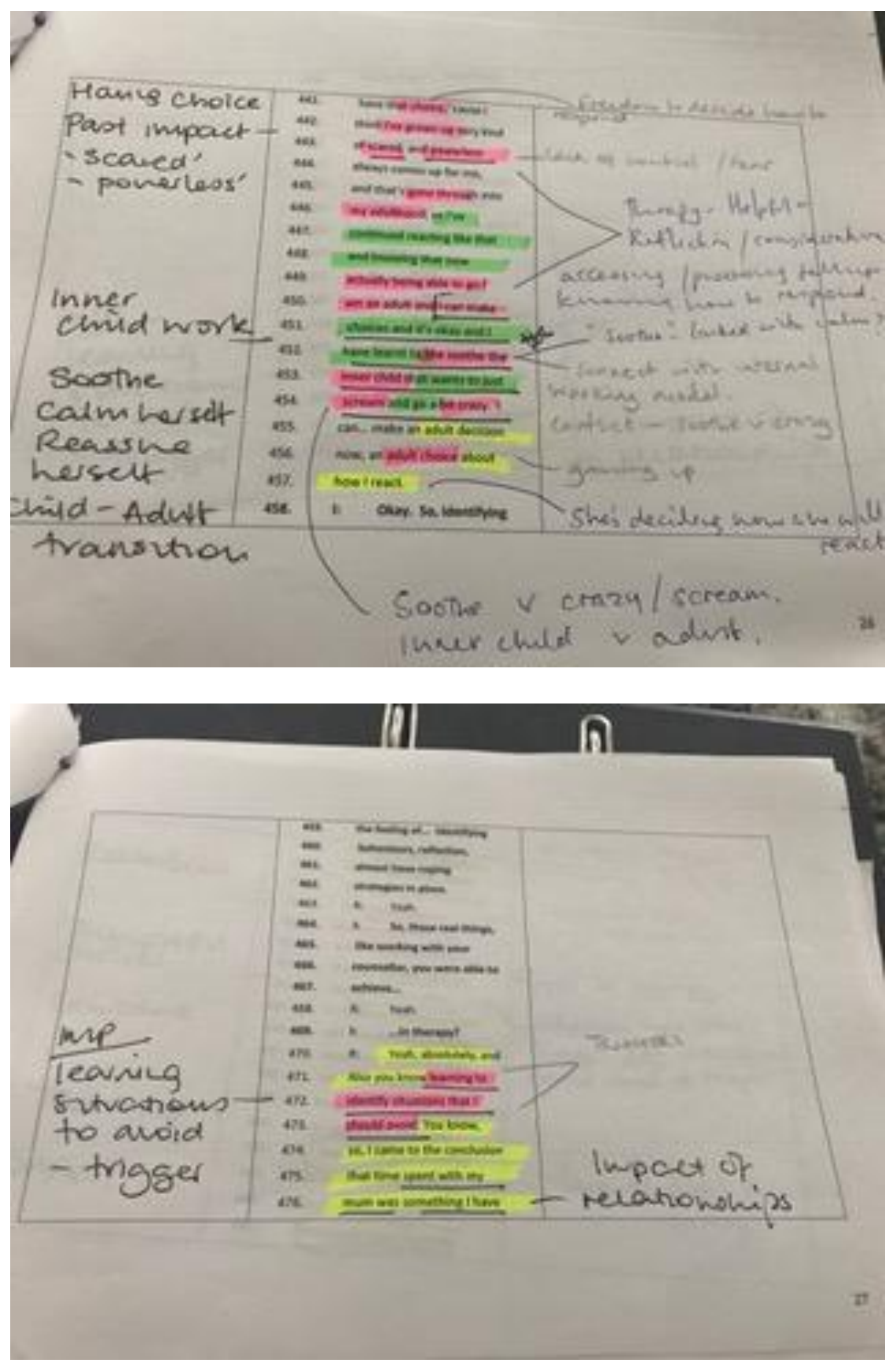
Helpful and unhelpful processes in psychological therapy for female substance users
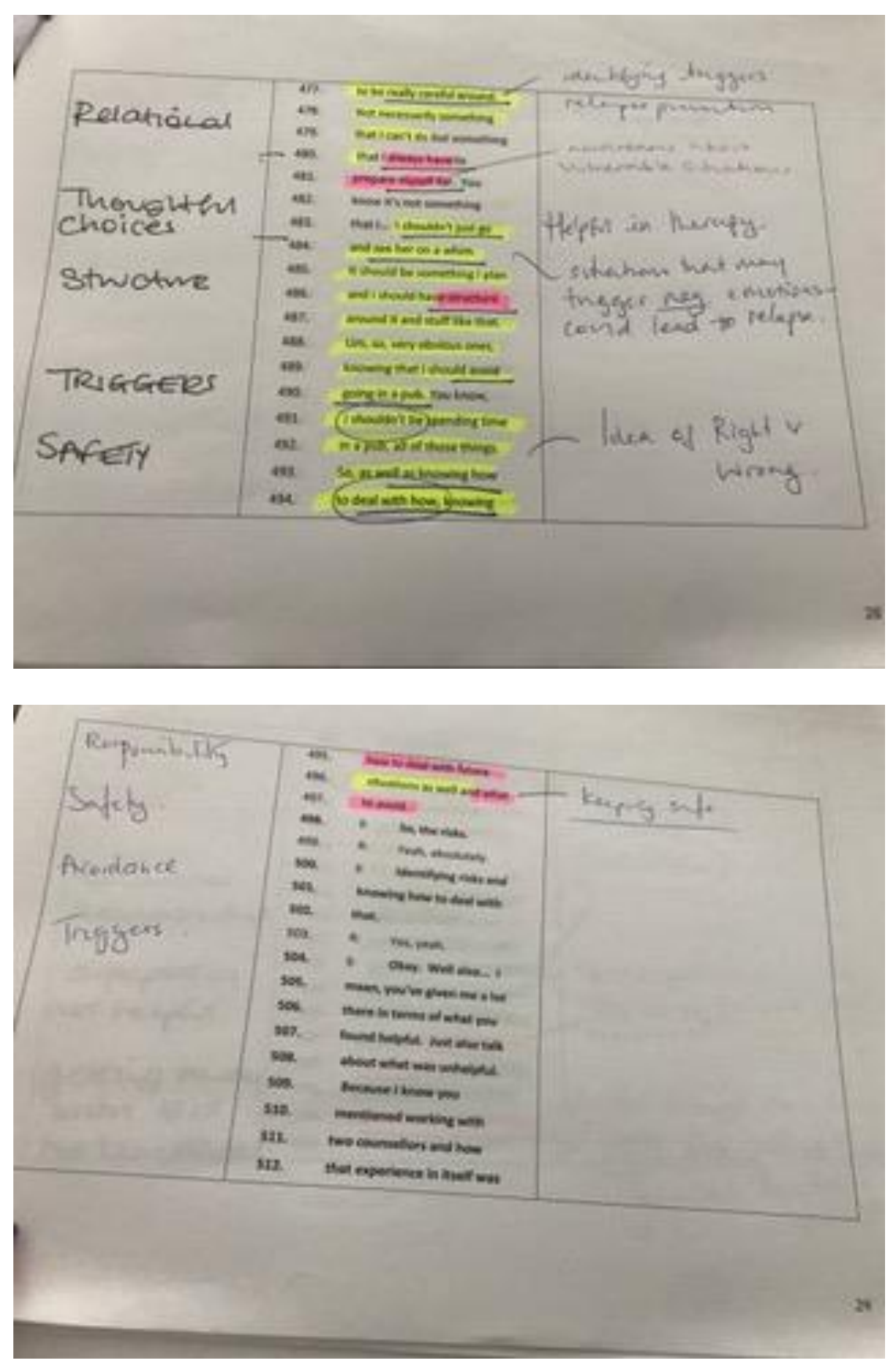
Helpful and unhelpful processes in psychological therapy for female substance users
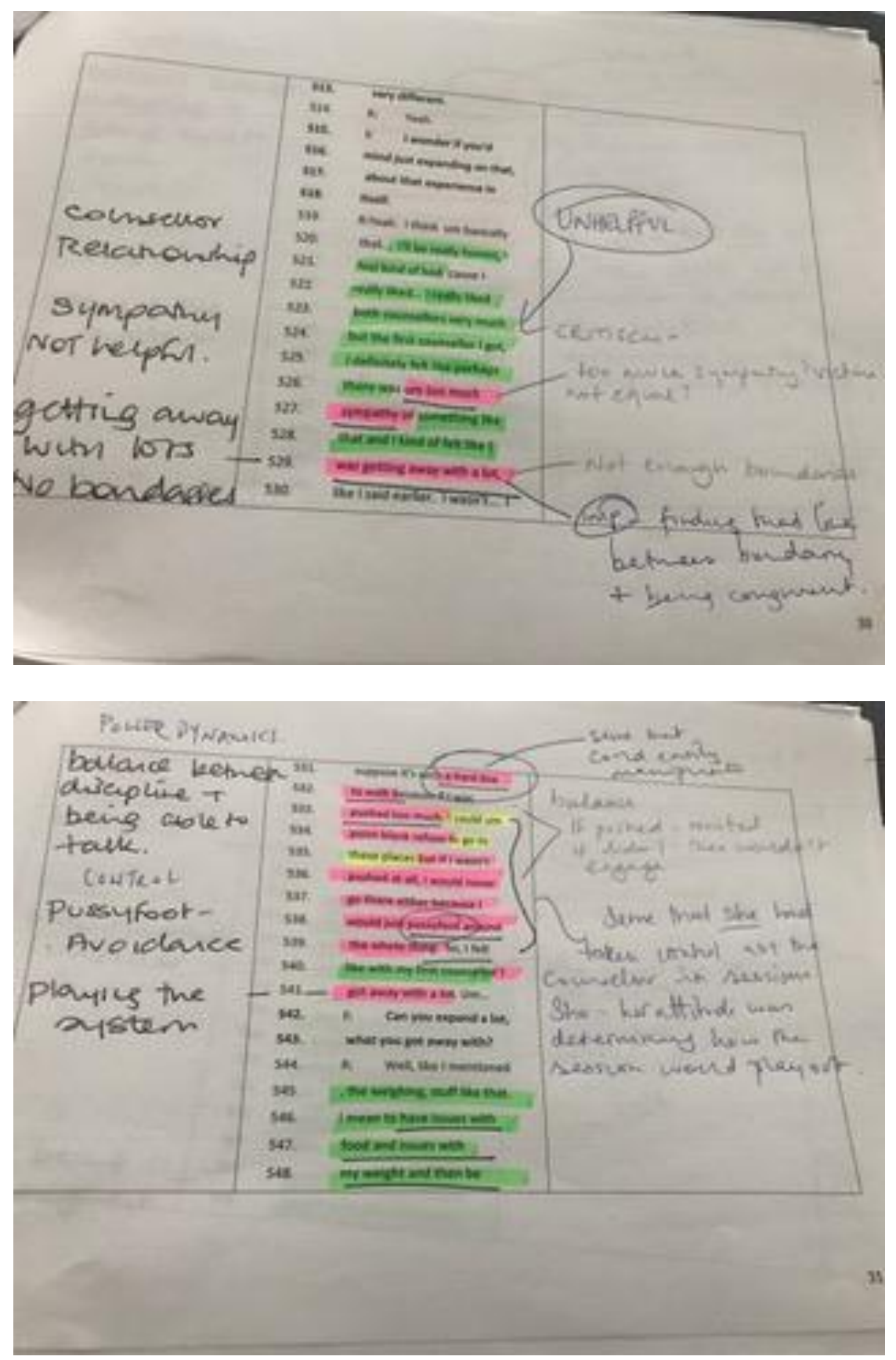
Helpful and unhelpful processes in psychological therapy for female substance users
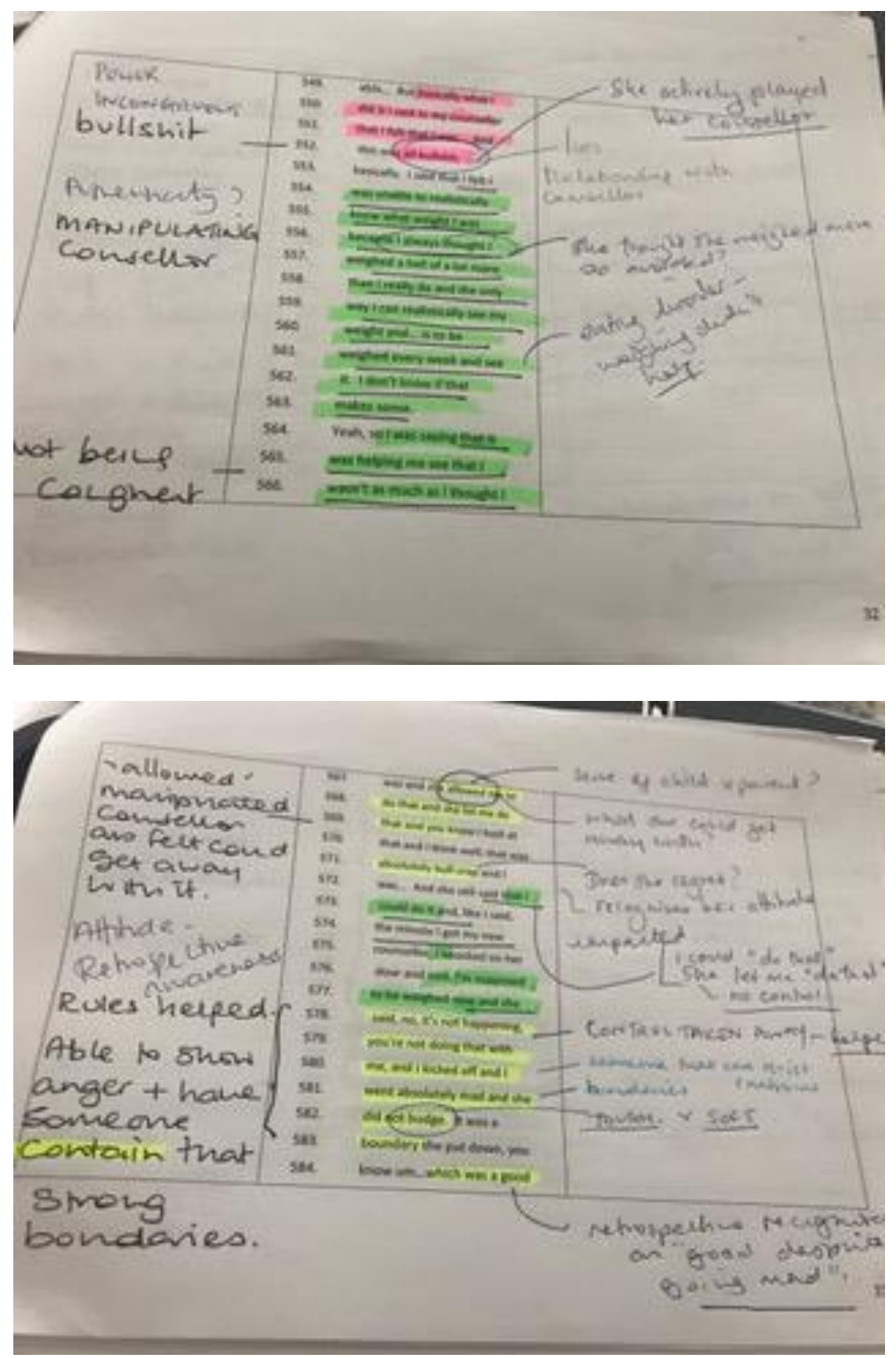
Helpful and unhelpful processes in psychological therapy for female substance users
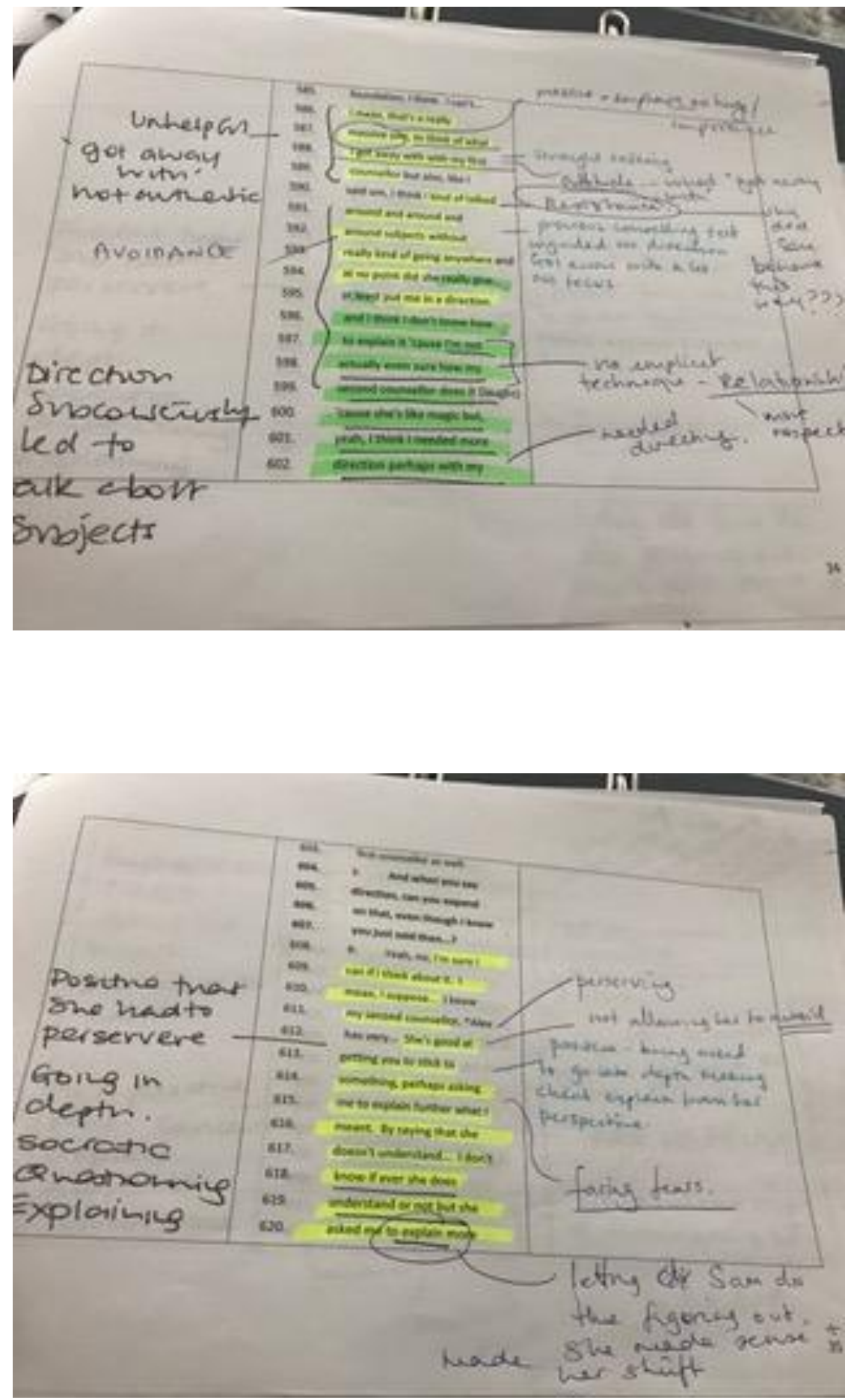
Helpful and unhelpful processes in psychological therapy for female substance users
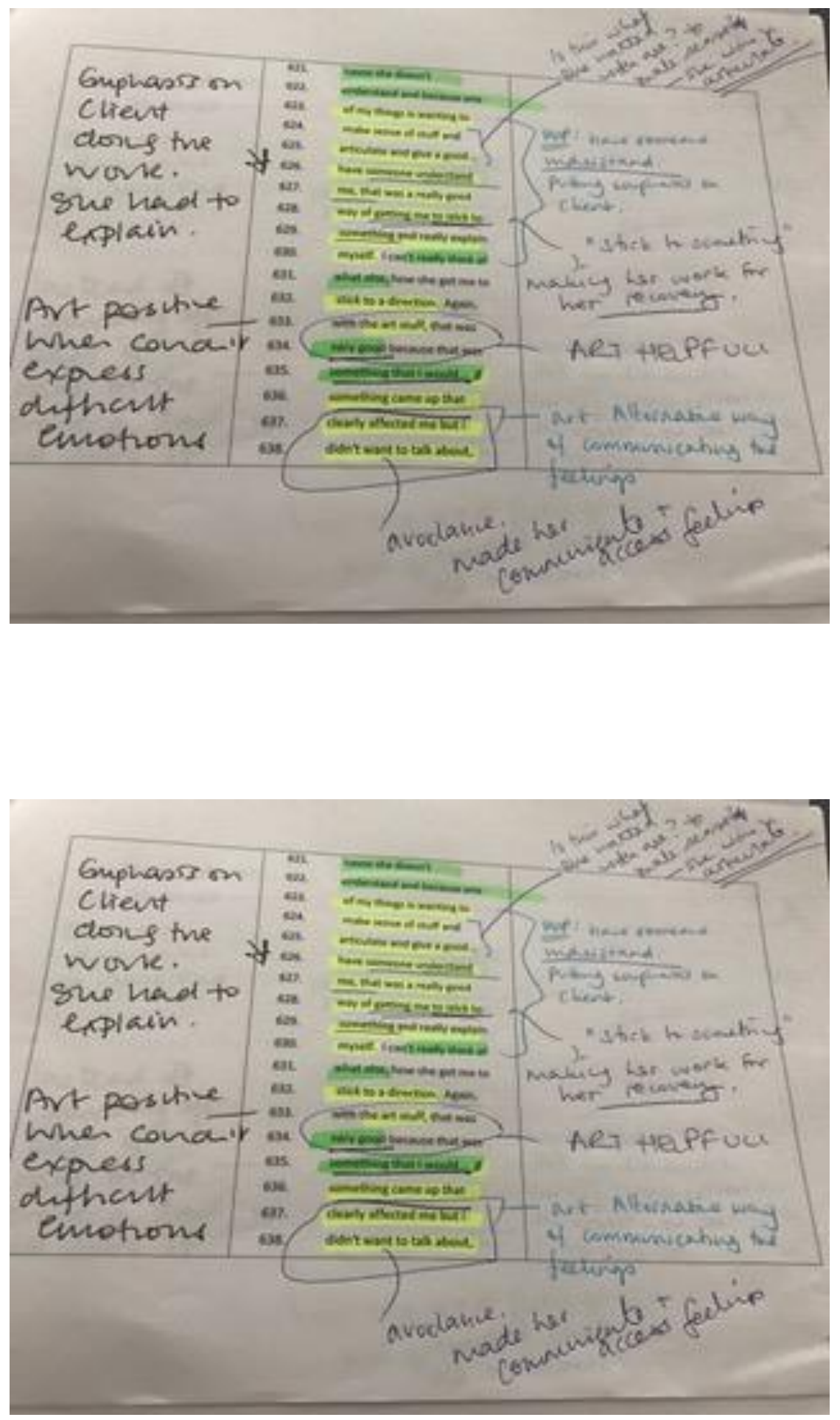
Helpful and unhelpful processes in psychological therapy for female substance users
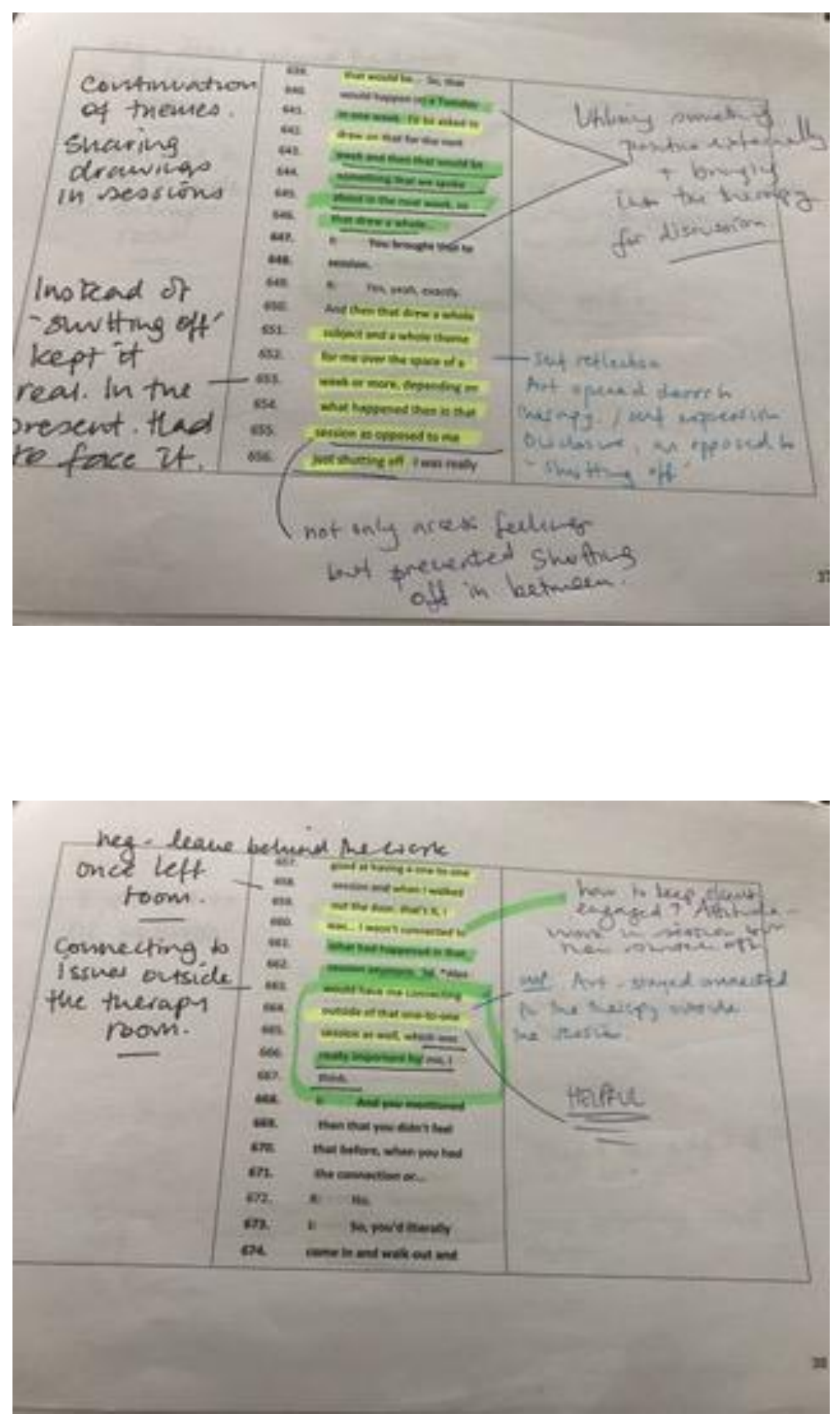
Helpful and unhelpful processes in psychological therapy for female substance users
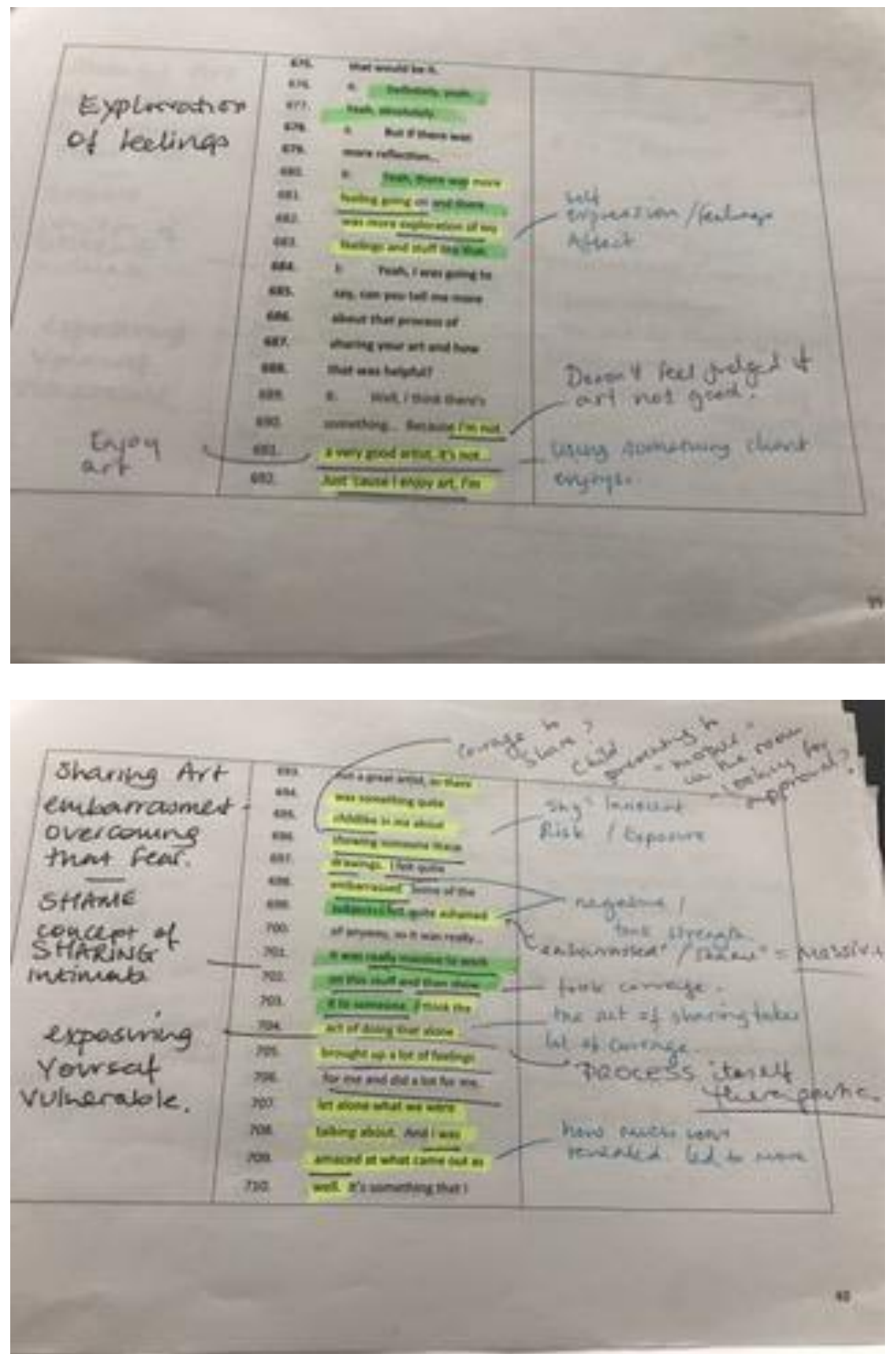
Helpful and unhelpful processes in psychological therapy for female substance users
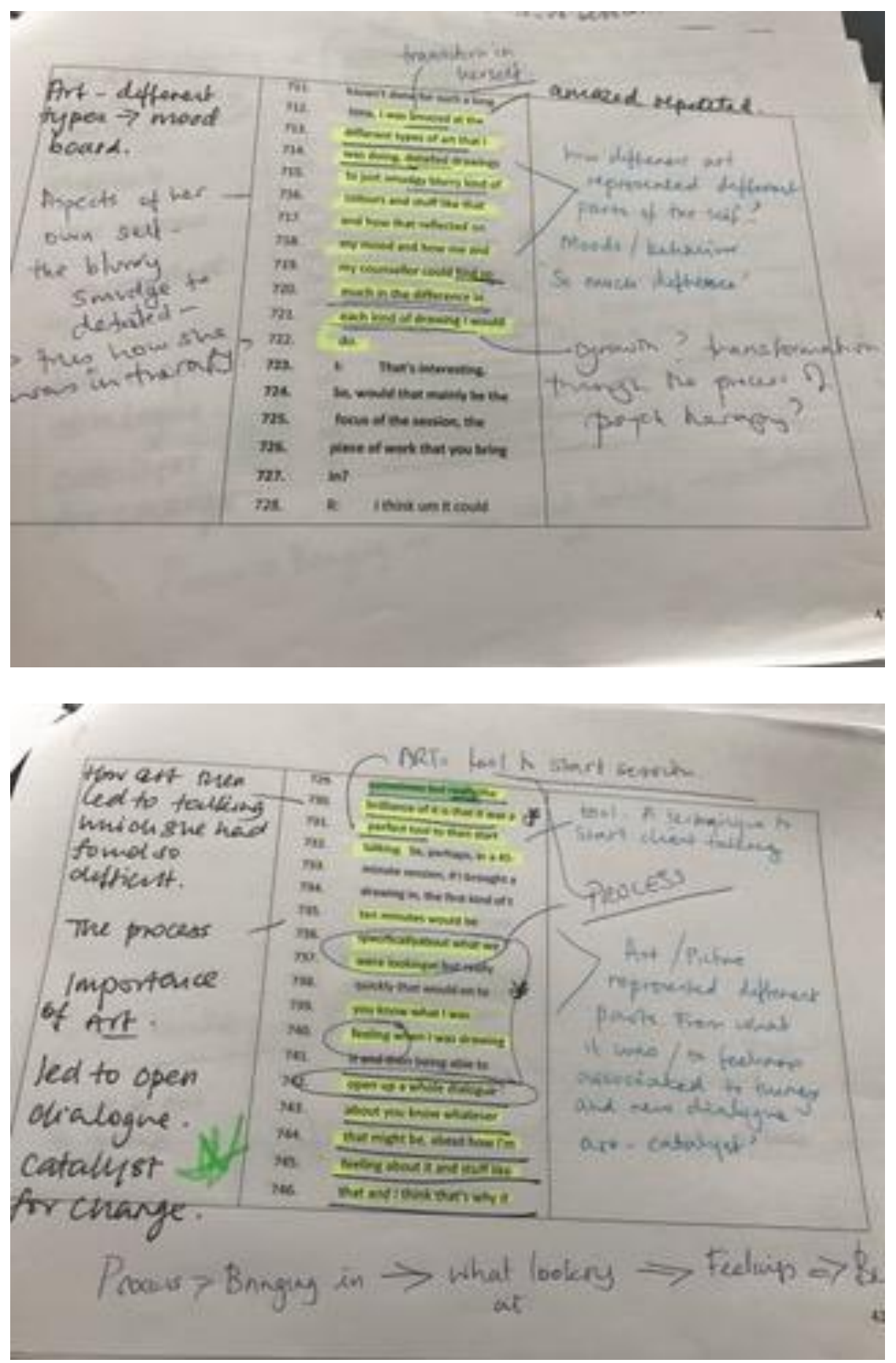
Helpful and unhelpful processes in psychological therapy for female substance users
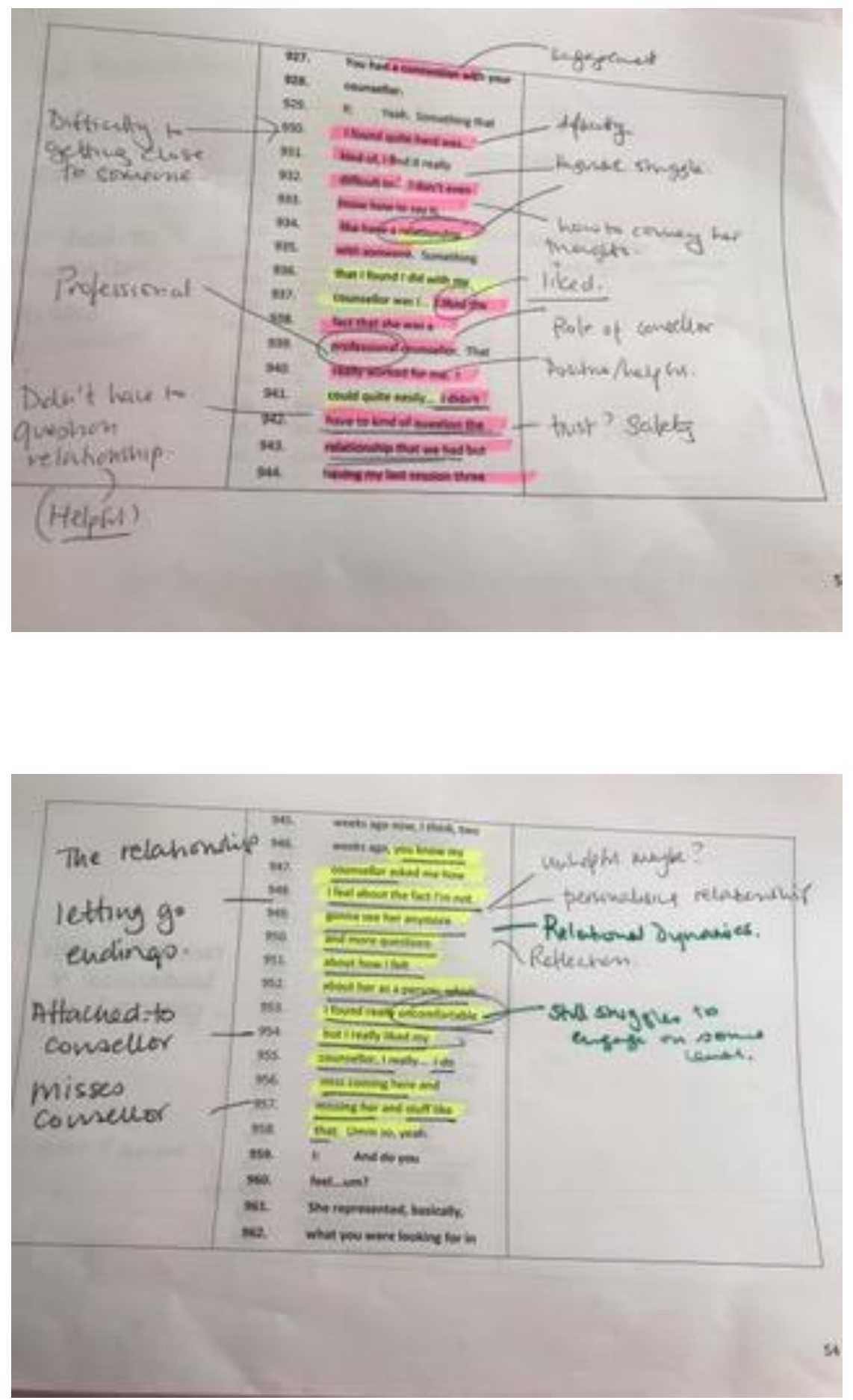
Helpful and unhelpful processes in psychological therapy for female substance users
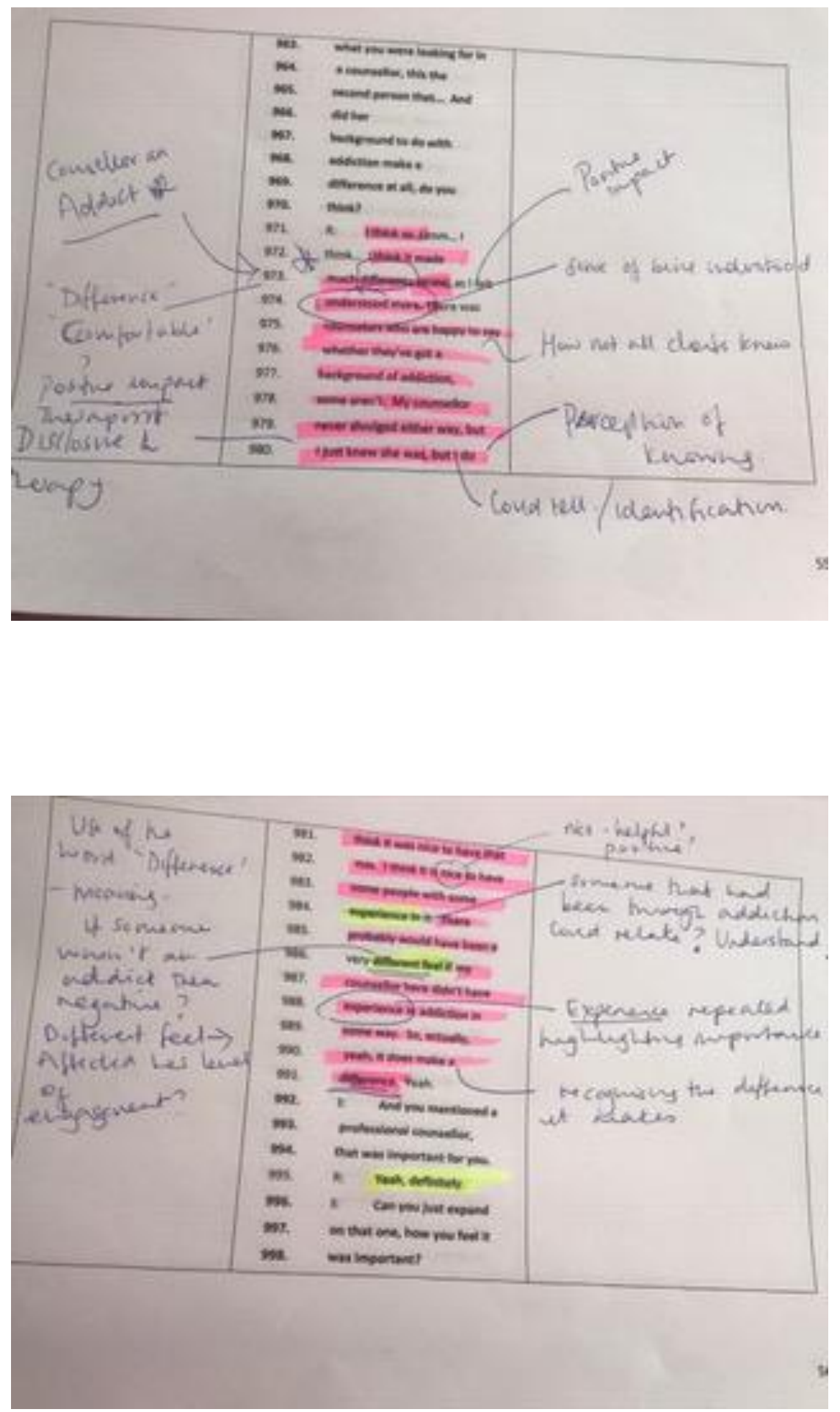
Helpful and unhelpful processes in psychological therapy for female substance users
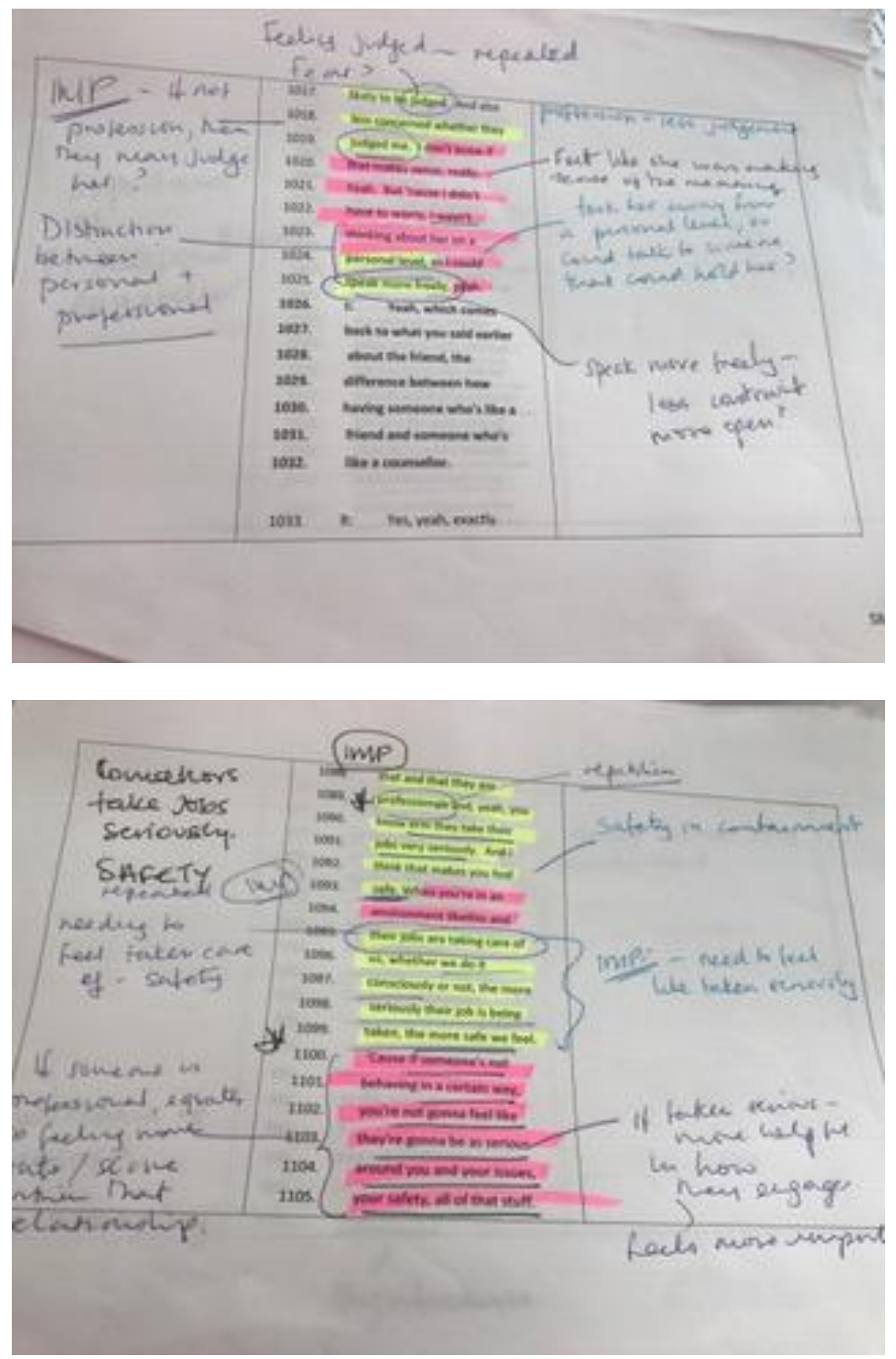
Helpful and unhelpful processes in psychological therapy for female substance users

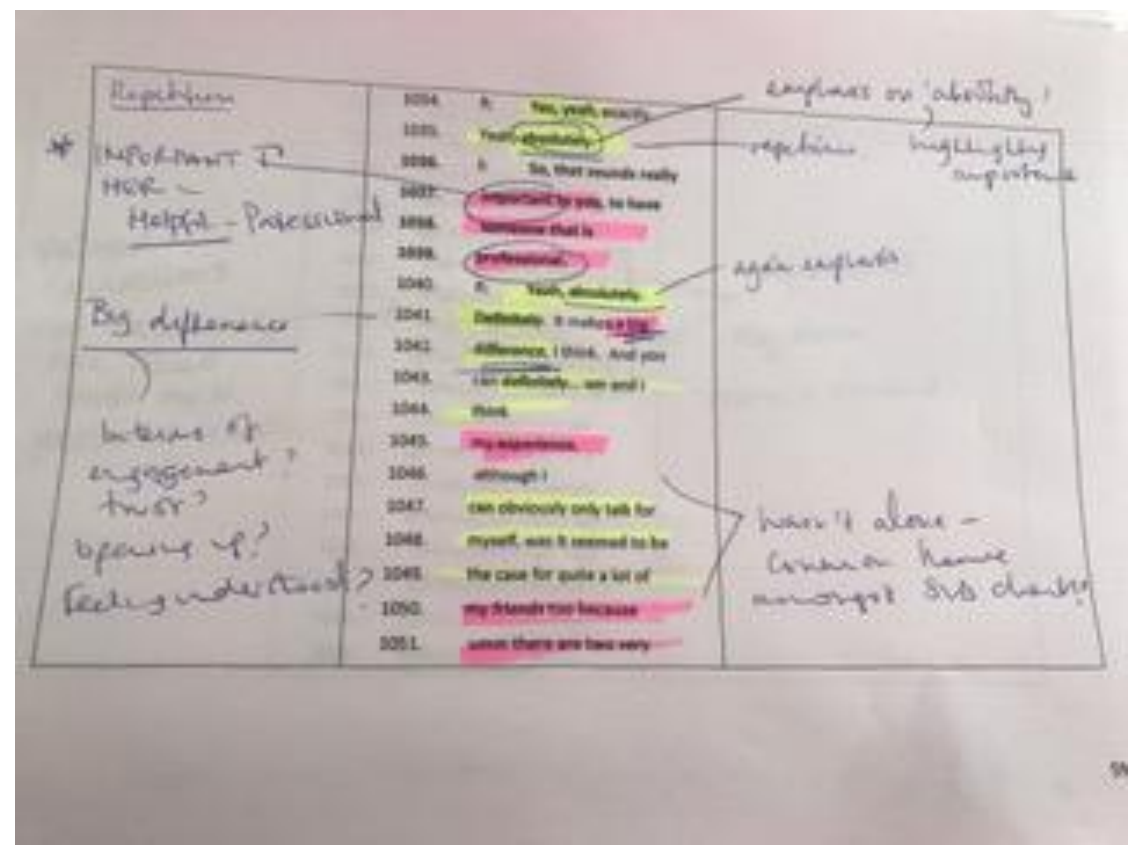


Helpful and unhelpful processes in psychological therapy for female substance users

$\frac{\text { EMERTENT }}{\text { THEMES }}$

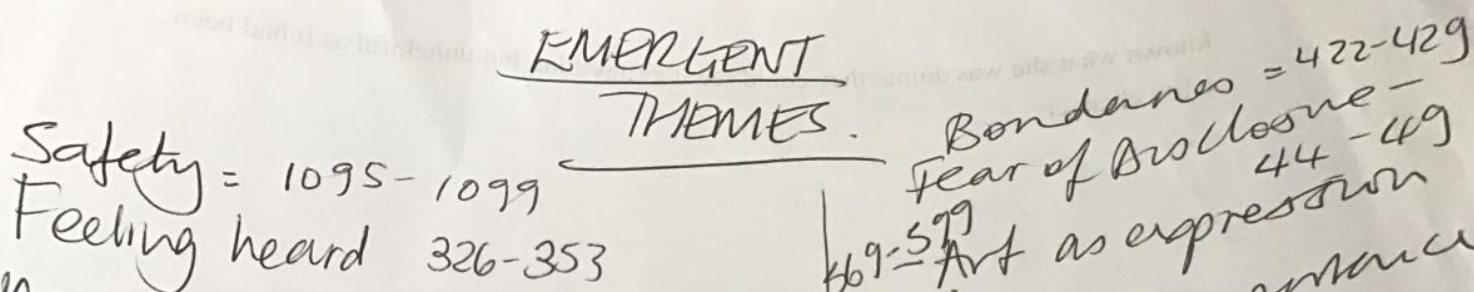
Feeling heard 326-353

Wore than a comellor - $197-199$ 569 - S Ant as expreson cotting trungh bulsut-1309-1322 Addict persona - 179-184 $85.12 \mathrm{R}^{3}$ sic - imponace ldeuhpeahen - 397-417

Defences $-28^{6}-29^{\circ}$

Regnet - 200-207

$147_{4} 0^{2}$ - letting go of he past

Acceplance- 557-560

545 -Expctarians of self

Sname-998-1025

Power Dynamics

Role of the consellor - 65-74

$511-$ Manip nation

Emergence of real seif - $123-140$ sitf Reflechon shoggle to find a voice $-49-51$
Fenr of beng judged $=123-134$
277
290 ${ }_{14} 7^{\circ}$-Copng wh change

profess conen equally sufety 253.25 fetf protection

Nomaling behanows - 204.209 fercephon v real

Relahoind empant of thergios Trust - 301-30 Accessus feelvines $-692-70^{3}$

challenges -64 suppressed emotons-246

Engagenert th sterventions in herapy Verary-90cettry go of he past - 277-

Hanls sonve 290

skilled-1:274

Appendix 10: Further workings of themes and quotes in tables

186 
Helpful and unhelpful processes in psychological therapy for female substance users

Participant 1: (SAM) INTEGRATIVE

Subordinate Theme: SELF EXPRESSION

\begin{tabular}{|c|c|c|}
\hline SUB THEME 1 & QUOTES & $\begin{array}{l}\text { Line } \\
\text { Numbers }\end{array}$ \\
\hline \multirow[t]{4}{*}{$\begin{array}{l}\text { Importance of non } \\
\text { verbal expression / } \\
\text { accessing feelings }\end{array}$} & $\begin{array}{l}\text { I am not a great artist, so there was } \\
\text { something quite childlike in showing } \\
\text { someone these drawings. I felt } \\
\text { embarrassed. Some of the subjects I felt } \\
\text { quite ashamed of anyone, so it was } \\
\text { really....it was massive to work on this } \\
\text { stuff and show to someone. }\end{array}$ & 692-703 \\
\hline & $\begin{array}{l}\text { I can really very quickly relate a colour to } \\
\text { a feeling rather than naming that feeling. I } \\
\text { find that so hard to name a feeling, its } \\
\text { really odd. Im far better at it now um } \\
\text { through working out what colours are } \\
\text { positive and negative for me and then } \\
\text { being able to link them to feelings has } \\
\text { now been able to help me identify my } \\
\text { feelings ' cause when I first came in, } \\
\text { being asked the question, how do you feel, } \\
\text { I was just lost. I would never know how to } \\
\text { pinpoint it. }\end{array}$ & $246-259$ \\
\hline & $\begin{array}{l}\text {...to choose a song that relates to a subject } \\
\text { and then play it can be quite scary and can } \\
\text { show a lot of you and say a lot more than } \\
\text { you ever could }\end{array}$ & 286-290 \\
\hline & $\begin{array}{l}\text { Being able to identify how I feel, like the } \\
\text { colours and stuff like that, makes such a } \\
\text { big difference because when I am } \\
\text { overwhelmed with an emotion, if I can't }\end{array}$ & $397-417$ \\
\hline
\end{tabular}




\begin{tabular}{|c|c|c|}
\hline & $\begin{array}{l}\text { put my finger on it, that's the void that I } \\
\text { can fill and want to fill with drugs cause I } \\
\text { don't know whats going on and its } \\
\text { almost..a kind of fear you know, fear } \\
\text { about what the hell am I feeling and then } \\
\text { you know I fill that with anything from } \\
\text { the outside I possibly can. So, the } \\
\text { immediate thing, being able to identify } \\
\text { how im feeling is massive. }\end{array}$ & \\
\hline \multirow[t]{2}{*}{ Emergence of real self } & $\begin{array}{l}\text { We kind of worked together to make these } \\
\text { links to why I was reacting the way I was, } \\
\text { yeah and exploring..i think that's } \\
\text { definitely worked for me }\end{array}$ & 338-342 \\
\hline & $\begin{array}{l}\text { Especially when I left aftercare....i } \\
\text { definitely, definitely saw that I had got } \\
\text { better and found it easier to share }\end{array}$ & 836-838 \\
\hline $\begin{array}{l}\text { Struggle in finding a } \\
\text { voice }\end{array}$ & $\begin{array}{l}\text { I really thought I was gonna be able to } \\
\text { come in and just talk and I couldn't. I } \\
\text { absolutely could not. } \\
\text { I felt that everything had to be censored, } \\
\text { everything had to be articulate and make } \\
\text { sense and I couldn't just talk. }\end{array}$ & $\begin{array}{l}143-145 \\
156-161\end{array}$ \\
\hline
\end{tabular}

Subordinate Theme: CUTTING THROUGH THE BULLSHIT / ATTITUDE TOWARDS COUNSELLING

\begin{tabular}{|l|l|l|}
\hline SUB THEME 2 & Quotes & $\begin{array}{l}\text { Line } \\
\text { Numbers }\end{array}$ \\
\hline Keeping it real & & \\
\hline & $\begin{array}{l}\text { I have learnt to soothe the inner child } \\
\text { that wants to scream and go a bit crazy. }\end{array}$ & $\mathbf{4 5 3 - 4 5 6}$ \\
\hline
\end{tabular}


Helpful and unhelpful processes in psychological therapy for female substance users

\begin{tabular}{|c|c|c|}
\hline & $\begin{array}{l}\text { I can... make an adult decision now, an } \\
\text { adult choice about how I react. }\end{array}$ & \\
\hline & $\begin{array}{l}\text { Ive really learnt not to consider too } \\
\text { much before I speak because generally, } \\
\text { if I do that, its not going to be as real }\end{array}$ & $163-167$ \\
\hline \multirow[t]{3}{*}{$\begin{array}{l}\text { Manipulation of the } \\
\text { counsellor }\end{array}$} & $\begin{array}{l}\text { I really wasn't talking about real stuff, I } \\
\text { was just kind of bullshitting around the } \\
\text { subject and avoiding the real issues. } \\
\text { Mostly subconsciously. I don't think I } \\
\text { really consciously put effort into doing } \\
\text { that but once I was with someone who } \\
\text { was a bit stricter, who had kind of more } \\
\text { boundaries and stuff like that...I almost } \\
\text { couldn't get away with that. Its like } \\
\text { when I was doing that, I really like felt } \\
\text { she could see that I was doing that.... }\end{array}$ & 87-100 \\
\hline & $\begin{array}{l}\text { She allowed me to do that and she let } \\
\text { me do that and you know I look at that } \\
\text { and I think well, that was absolutely bull } \\
\text { crap }\end{array}$ & $567-569$ \\
\hline & $\begin{array}{l}\text { The first counselor I got, I definitely felt } \\
\text { like perhaps there was um too much } \\
\text { sympathy or something like that and I } \\
\text { felt I was getting away with a lot } \\
\text { I mean, that's a really massive one, to } \\
\text { think of what I got away with with my } \\
\text { first counselor but also, like I said um, I } \\
\text { think I kind of talked around and around } \\
\text { and around subjects without really kind } \\
\text { of going anywhere and at no point did } \\
\text { she really ... at least put me in a } \\
\text { direction }\end{array}$ & 586-595 \\
\hline
\end{tabular}


Helpful and unhelpful processes in psychological therapy for female substance users

\begin{tabular}{|c|c|c|}
\hline Feeling understood & $\begin{array}{l}\text { I think learning to trust someone, } \\
\text { learning to...not feel judged meant that } \\
\text { I could..i began to be able to talk } \\
\text { more... }\end{array}$ & 179-184 \\
\hline & $\begin{array}{l}\text { By sayng she doesn't understand...I } \\
\text { don't know if she ever does understand } \\
\text { or not but she asked me to explain more } \\
\text { cause she doesn't understand and } \\
\text { because one of my things is wanting to } \\
\text { make sense of stuff and articulate and } \\
\text { give a good..have someone understand } \\
\text { me, that was a really good way of } \\
\text { getting me to stick to something and } \\
\text { really explain myself }\end{array}$ & 612-630 \\
\hline
\end{tabular}

\begin{tabular}{|c|c|c|}
\hline \multicolumn{3}{|l|}{ Professional v Personal } \\
\hline & $\begin{array}{l}\text { I changed my counsellor very quickly she } \\
\text { was like, no, that's not happening... she was } \\
\text { a lot stricter... and that really helped me. I } \\
\text { didn't need a friend as much as a counsellor } \\
\text { and to really feel like I was in therapy } \\
\text { maybe. }\end{array}$ & $65-74$ \\
\hline & $\begin{array}{l}\text { Something that I found I did like with my } \\
\text { counsellor was i...I liked the fact she was a } \\
\text { professional counsellor. That really worked } \\
\text { for me..... } \\
\text { I think the reason it was important for me } \\
\text { was because I was...im sharing about stuff } \\
\text { that I felt a lot of shame about and If I felt } \\
\text { like I was being...if I felt like I was talking } \\
\text { to someone on a personal level, my fears }\end{array}$ & $\begin{array}{l}\text { 937- } \\
939 \\
\\
\\
\text { 998- } \\
\mathbf{1 0 2 5}\end{array}$ \\
\hline
\end{tabular}


Helpful and unhelpful processes in psychological therapy for female substance users

\begin{tabular}{|c|c|c|}
\hline & $\begin{array}{l}\text { around being judged would really come into } \\
\text { play and would potentially stop me from } \\
\text { talking about what I needed to talk about. } \\
\text { The feeling I was talking to someone who } \\
\text { was a professional, who knew what they } \\
\text { were doing in terms of counselling and that } \\
\text { kind of thing, made me feel less likely to be } \\
\text { judged. } \\
\text { When your in an environment like this and } \\
\text { their jobs are taking care of us, whether we } \\
\text { do it consciously or not, the more seriously } \\
\text { their job is being taken, the more safe we } \\
\text { feel. }\end{array}$ & $\begin{array}{l}1095 \\
1099\end{array}$ \\
\hline \multirow[t]{2}{*}{ Addict Identification } & $\begin{array}{l}\text { It felt different when I knew my counsellor } \\
\text { was the same as us, as in the rooms. Not } \\
\text { everyone's experience is the same, but she } \\
\text { made me see there is another side, there is } \\
\text { hope. }\end{array}$ & 341 \\
\hline & $\begin{array}{l}\text { I think it is nice to have some people with } \\
\text { some experience in it. There probably } \\
\text { would have been a very different feel if } \\
\text { they didn't have experience in addiction in } \\
\text { some way. }\end{array}$ & $\begin{array}{l}\text { 981- } \\
989\end{array}$ \\
\hline
\end{tabular}

PARTICIPANT 2: (ANITA) PSYCHODYNAMIC

SUBORDINATE THEME: Fear of opening up /shame

\begin{tabular}{|l|l|l|}
\hline SUB THEME 1: & QUOTE & $\begin{array}{l}\text { LINE } \\
\text { NUMBER }\end{array}$ \\
\hline
\end{tabular}


Helpful and unhelpful processes in psychological therapy for female substance users

\begin{tabular}{|c|c|c|}
\hline \multirow[t]{3}{*}{ Fear of disclosure } & $\begin{array}{l}\text { Ive never allowed anyone in, in my whole } \\
\text { childhood, and really up until sort of...I've } \\
\text { started to let go now, but to be able to sit } \\
\text { with someone and tell... say to them how } \\
\text { I'm feeling, I really did struggle with that. }\end{array}$ & 44-49 \\
\hline & $\begin{array}{l}\text { I just couldn't understand why I had to talk } \\
\text { about it. I thought I just need to block and } \\
\text { they'd go off. I'd go in there. I thought, } \\
\text { why do I have to go there? } \\
\text { Why... why... because it's painful. But I } \\
\text { didn't realise how important it is to go } \\
\text { there, to free yourself up from that....from } \\
\text { that pain. Where I would keep the pain. }\end{array}$ & $428-436$ \\
\hline & $\begin{array}{l}\text { I could talk about the suicide attempts, and } \\
\text { I've had three. And you know. I don't } \\
\text { know, it got brought up in groups, but I } \\
\text { wouldn't go there, but I know now I need } \\
\text { to go there. I need to understand what } \\
\text {....where I was then and what was going } \\
\text { on for me. }\end{array}$ & $587-595$ \\
\hline $\begin{array}{l}\text { Comparing to others / } \\
\text { art }\end{array}$ & $\begin{array}{l}\text { I found it hard to bring stuff in myself, } \\
\text { because I didn't think it was good enough, } \\
\text { that's why art helped me. It was personal } \\
\text { but meant so much to have another outlet. I } \\
\text { don't think my drawings were any good, } \\
\text { but they were used in my sessions. Not all } \\
\text { the time, but when I really was struggling } \\
\text { to open up they helped. Especially when I } \\
\text { couldn't find the words }\end{array}$ & 181-183 \\
\hline Letting go of the past & $\begin{array}{l}\text { I know the role I played, and I look at how } \\
\text { my childhood was and what I experienced, }\end{array}$ & $511-516$ \\
\hline
\end{tabular}


Helpful and unhelpful processes in psychological therapy for female substance users

\begin{tabular}{|c|c|c|}
\hline & $\begin{array}{l}\text { but again it's gone, and part of me is like, } \\
\text { do I really want to go there? }\end{array}$ & \\
\hline & $\begin{array}{l}\text { I've never been able to get vulnerable and } \\
\text { it's never been acceptable for me to be like } \\
\text { that. }\end{array}$ & \\
\hline Resistance & $\begin{array}{l}\text { I thought why do I have to go there? } \\
\text { Why..why...because it's painful. But I } \\
\text { didn't realise how important it is to go } \\
\text { there, to free yourself up from that...from } \\
\text { that pain. }\end{array}$ & $430-436$ \\
\hline
\end{tabular}

SUBORDINATE THEME: Therapeutic relationship

\begin{tabular}{|c|c|c|}
\hline SUB THEME 2 & Quote & $\begin{array}{l}\text { Line } \\
\text { Number }\end{array}$ \\
\hline \multirow[t]{2}{*}{$\begin{array}{l}\text { Feeling let down - } \\
\text { unhelpful }\end{array}$} & $\begin{array}{l}\text { I do feel that I could have been pushed } \\
\text { more, for the person that I am }\end{array}$ & 69-71 \\
\hline & $\begin{array}{l}\text { Maybe known a bit more about me before } \\
\text { my counselling started. }\end{array}$ & 644-645 \\
\hline \multirow[t]{3}{*}{ Manipulation } & $\begin{array}{l}\text { Because I controlled the situation. Maybe } \\
\text { someone to tell me how it is, and let me } \\
\text { go, well actually your right. And then } \\
\text { maybe I might have opened up more. But } \\
\text { I just felt I was mothered too much. }\end{array}$ & 110-116 \\
\hline & $\begin{array}{l}\text { I felt that I controlled my one to ones. } \\
\text { There was a situation }\end{array}$ & \\
\hline & $\begin{array}{l}\text { My character defects are my } \\
\text { perfectionism, and my controlling. And I } \\
\text { need...I need someone whose...I don't } \\
\text { say that she doesn't understand because I } \\
\text { know shes got experience, but I just...I }\end{array}$ & $124-133$ \\
\hline
\end{tabular}


Helpful and unhelpful processes in psychological therapy for female substance users

\begin{tabular}{|c|c|c|}
\hline & $\begin{array}{l}\text { felt I was the clever one. And I don't } \\
\text { think that did me any justice. }\end{array}$ & \\
\hline & $\begin{array}{l}\text { My body language, how I could change } \\
\text { the subject, um again, I manipulated the } \\
\text { thirty minute silence. I survive; if it sits } \\
\text { right with you, it sits right with me. }\end{array}$ & 576-580 \\
\hline $\begin{array}{l}\text { Impact of counsellor / } \\
\text { safety }\end{array}$ & $\begin{array}{l}\text { But then, that trust issue is from } \\
\text { childhood and I'm aware of that, and I } \\
\text { think with therapists, I just know that } \\
\text { they're trained. They're trained, which } \\
\text { means that you know, theres } \\
\text { confidentiality. You know, there's a } \\
\text { framework. }\end{array}$ & 301-305 \\
\hline \multirow{3}{*}{$\begin{array}{l}\text { Addict Persona / } \\
\text { identification }\end{array}$} & It made a massive difference & 968 \\
\hline & $\begin{array}{l}\text { There were some things I would get } \\
\text { embarrassed about....now I know you've } \\
\text { been through it now, it was more } \\
\text { comfortable I suppose, yeah }\end{array}$ & $955-960$ \\
\hline & $\begin{array}{l}\text { Because they can feel it. It's like they } \\
\text { understand. It's not so shameful. It's not } \\
\text { so shameful. It's like they're telling me it } \\
\text { is ok; it's alright }\end{array}$ & $907-911$ \\
\hline
\end{tabular}

SUBORDINATE THEME: RETROSPECTIVE REFLECTION /

EMERGENCE OF NEW SELF

\begin{tabular}{|l|l|l|}
\hline Sub Theme 3 & Quote & $\begin{array}{l}\text { Line } \\
\text { number }\end{array}$ \\
\hline Regret & $\begin{array}{l}\text { Looking back now, if I could change it I } \\
\text { would. I would. I'd loved to have been } \\
\text { more open and honest. }\end{array}$ & $\mathbf{5 5 4 - 5 5 6}$ \\
\hline
\end{tabular}


Helpful and unhelpful processes in psychological therapy for female substance users

\begin{tabular}{|l|l|l|}
\hline Acceptance & $\begin{array}{l}\text { Rather than go into self-pity, I can } \\
\text { accept that this happened. I wanna be } \\
\text { free for it, and I think if I talk about it, I } \\
\text { can build myself up from it }\end{array}$ & $\mathbf{8 5 1 - 8 5 5}$ \\
\hline $\begin{array}{l}\text { But maybe I needed to go through that } \\
\text { to be where I am now to understand the } \\
\text { type of person that I am. }\end{array}$ & $\mathbf{5 5 7 - 5 6 0}$ \\
\hline Expectations & $\begin{array}{l}\text { I thought I was gonna come in and get } \\
\text { cured and this was gonna happen to me, } \\
\text { and it wasn't happening to me. }\end{array}$ & \\
\hline
\end{tabular}

\section{PARTICIPANT 3 : (EMMA) INTEGRATIVE}

\section{SUBORDINATE THEME: process of change?}

\begin{tabular}{|c|c|c|}
\hline Subtheme 1: & Quote & Line \\
\hline \multirow[t]{2}{*}{ Letting go of the past } & $\begin{array}{l}\text { I guess, I guess that a lot of guilt and } \\
\text { shame came from, you know, my feelings } \\
\text { starting to kick in; the realisation side of } \\
\text { things. I'd actually done, justified...that I } \\
\text { couldn't justify anymore. Um and I kind } \\
\text { of..part of me that a lot... a big part of me } \\
\text { just thought that this was the path I was } \\
\text { on. I was destined to beat myself and that } \\
\text { for others to beat me for the rest of my } \\
\text { days. }\end{array}$ & $277-290$ \\
\hline & $\begin{array}{l}\text { I didn't fully appreciate I was an addict of } \\
\text { any sort. I just thought this was the way it } \\
\text { was going to be. That's my life. }\end{array}$ & 17-19 \\
\hline Art / music & $\begin{array}{l}\text { I didn't think I would be able to talk about } \\
\text { everything ive been through. That's why } \\
\text { art and music were really good for } \\
\text { me....they could let her know how I felt. }\end{array}$ & $\begin{array}{l}1470- \\
1492\end{array}$ \\
\hline
\end{tabular}


Helpful and unhelpful processes in psychological therapy for female substance users

\begin{tabular}{|c|c|c|}
\hline & $\begin{array}{l}\text { It was quite ground breaking for me to be } \\
\text { able to share...I heard her voice say 'Just } \\
\text { identify how your feeling' and I sat there. } \\
\text { I was thinking I'm okay. I'm in this place } \\
\text { that ive avoided, because it holds all the } \\
\text { memories of crap, but actually im okay. I } \\
\text { don't wanna use, I don't wanna scream, } \\
\text { cry... um... and then I think that's when I } \\
\text { started to think actually I can...I can do } \\
\text { this. I can do this recovery stuff, you } \\
\text { know. }\end{array}$ & \\
\hline \multirow[t]{3}{*}{ containment } & $\begin{array}{l}\text { I kept saying to her like 'Look, ive been } \\
\text { using since I was ten, and If I do let go, } \\
\text { you know, quite angrily, if you keep } \\
\text { pushing me, and you break me' because it } \\
\text { was all about her breaking me (laughs), 'if } \\
\text { you break me, you knowthen im gonna } \\
\text { cry and have screaming fits for days, } \\
\text { weeks; your not gonna be able to hold it. }\end{array}$ & $461-473$ \\
\hline & $\begin{array}{l}\text { She showed me she wasn't scared of my } \\
\text { anger, that is was ok to be angry, it was } \\
\text { ok to be upset. }\end{array}$ & $\begin{array}{l}\text { 1008- } \\
1011\end{array}$ \\
\hline & $\begin{array}{l}\text { Yeah, her enforcing those boundaries was } \\
\text { like, brilliant boundaries and brilliant } \\
\text { morals with my children, always have } \\
\text { somehow saved me, I guess; not just } \\
\text { them, but me. } \\
\text { I needed that, you know. I needed to learn } \\
\text { boundaries. I still reinforce boundaries } \\
\text { with myself now. }\end{array}$ & $\begin{array}{l}1182- \\
1185\end{array}$ \\
\hline
\end{tabular}

SUBORDINATE THEME: COUNSELLOR DYNAMIC 
Helpful and unhelpful processes in psychological therapy for female substance users

\begin{tabular}{|c|c|c|}
\hline Subtheme 2 & Quote & Line \\
\hline $\begin{array}{l}\text { Manipulation of the } \\
\text { counsellor and how she } \\
\text { knew. }\end{array}$ & $\begin{array}{l}\text { Um...just those first two sessions. I } \\
\text { remember them so clearly, and that I was } \\
\text { just so angry with her for...for..you } \\
\text { know, I thought she just not listening. } \\
\text { Yeah im being really honest here, shes } \\
\text { not listening... } \\
\text { I think its... }\end{array}$ & 423-431 \\
\hline Role of counsellor & $\begin{array}{l}\text { I didn't need a friend as much as a } \\
\text { counsellor and to feel like I was in } \\
\text { therapy }\end{array}$ & 75 \\
\hline \multirow[t]{2}{*}{ Addict affiliation } & $\begin{array}{l}\text { You know I was quite cocky because I } \\
\text { trained in counselling myself, so my } \\
\text { counsellor, bless her, she had a hard job } \\
\text { with me. She'd ask me how I was feeling } \\
\text { and I'd ask her how she was feeling, and } \\
\text { I'd say things to shock her. I'd just } \\
\text { randomly come out with something, that } \\
\text { was true, but would be a shock tactic. } \\
\text { And then I'd ask her how she was } \\
\text { feeling, so there was a lot of } \\
\text { um...defence on my side...but it did help } \\
\text { the fact that I knew she was in recovery: } \\
\text { that really made a difference. I actually } \\
\text { found that out halfway through treatment, } \\
\text { and when I did I thought...you know. } \\
\text { She knows (laughs) }\end{array}$ & 199-222 \\
\hline & $\begin{array}{l}\text { This woman was, you know, together and } \\
\text { lovely, and everything I didn't know an } \\
\text { addict could have. }\end{array}$ & $290-295$ \\
\hline Feeling heard & $\begin{array}{l}\text { I've had experience of counselling, } \\
\text { shrinks and all sorts, right from when I } \\
\text { was little, and every time I open my }\end{array}$ & 326-353 \\
\hline
\end{tabular}


Helpful and unhelpful processes in psychological therapy for female substance users

\begin{tabular}{|c|c|c|}
\hline & $\begin{array}{l}\text { mouth to tell them something that I really } \\
\text { needed someone to hear, they'd kind of } \\
\text { go to something completely different and } \\
\text { I'd feel unheard..... So when people sort } \\
\text { of, you know, particularly the } \\
\text { counsellors, when they spoke about their } \\
\text { own feelings, and, you know, that kind of } \\
\text { put a human on it; and they were actually } \\
\text { talking to me, and not like a study but a } \\
\text { human being that's hurt and trying to get } \\
\text { well. }\end{array}$ & \\
\hline $\begin{array}{l}\text { More than just a } \\
\text { counsellor }\end{array}$ & $\begin{array}{l}\text { So she had this role as some sort of } \\
\text { family member, female family member, } \\
\text { who I didn't live with, who was kind of } \\
\text { there, but im off to uni or whatever, you } \\
\text { know. It was that kind of connection. }\end{array}$ & $510-518$ \\
\hline Cut through the bullshit & $\begin{array}{l}\text { Yeah Mels* always been upfront and } \\
\text { open about her addictions, and you know, } \\
\text { shes just a beautiful graceful, intelligent } \\
\text { woman, whose stuck needles in places } \\
\text { where I have, and been to depths where I } \\
\text { have. Um, and she'll just kind of... she } \\
\text { just has this way of looking at you and } \\
\text { going 'I know what your thinking and } \\
\text { feeling, don't try and deny nothing with } \\
\text { me because I just told you. I just told you } \\
\text { that ive been there, done that, so now try } \\
\text { and tell me different.' }\end{array}$ & $\begin{array}{l}1309- \\
1322\end{array}$ \\
\hline
\end{tabular}

SUBORDINATE THEME: THE IMPORTANCE OF DIFFERENT FORMS OF SELF EXPRESSION 
Helpful and unhelpful processes in psychological therapy for female substance users

\begin{tabular}{|c|c|c|}
\hline ART & $\begin{array}{l}\text { But she encouraged me to do artwork. That } \\
\text { was quite interesting; that's something I still } \\
\text { do actually. }\end{array}$ & $\begin{array}{l}596- \\
599\end{array}$ \\
\hline & $\begin{array}{l}\text { Um...I guess its just um....see sometimes, } \\
\text { with a normal conversation in... with my } \\
\text { counsellor i can kind of go off in a direction, } \\
\text { and sometimes it'll be because its too painful. } \\
\text { Sometimes its just where my head wanders. } \\
\text { When you do that with art, you know, when } \\
\text { you do that in conversation, you know, when } \\
\text { you finish talking in a painful conversation, } \\
\text { there's a painful silence; when you do it with } \\
\text { art, there's a product infront of you that } \\
\text { collates all that, you know...good times and } \\
\text { bad times... whatever that may be. }\end{array}$ & $\begin{array}{l}685- \\
703\end{array}$ \\
\hline
\end{tabular}

PARTICIPANT 4 (KAT) INTEGRATIVE

\section{SUBORDiNATE THEME: THE DEVELOPMENT OF THE RECOVERING SELF / ATTITUDE}

\begin{tabular}{|l|l|l|}
\hline Subtheme 1 & Quote & Line \\
\hline Unable to talk & $\begin{array}{l}\text { I've not been clean or sober in years. So } \\
\text { when I left * I thought I was well and then } \\
\text { when I got here I got a fear, I couldn't even } \\
\text { speak. }\end{array}$ & $\mathbf{2 5 - 2 8}$ \\
\hline Resistance & $\begin{array}{l}\text { At first I didn't want to be here because I had } \\
\text { no boundaries and I had no structure....in the } \\
\text { beginning I was a bit, oh god, I'm just goint } \\
\text { to be one of the addicts out there }\end{array}$ & $\begin{array}{l}\mathbf{6 5 1 -} \\
\mathbf{6 5 5}\end{array}$ \\
\hline
\end{tabular}


Helpful and unhelpful processes in psychological therapy for female substance users

\begin{tabular}{|c|c|c|}
\hline Soothing the new self & $\begin{array}{l}\text { I used to always tell myself I shouldn't...I } \\
\text { wasn't allowing myself or honouring my } \\
\text { feelings and now im like, you know what, it's } \\
\text { ok to feel that. }\end{array}$ & \\
\hline & $\begin{array}{l}\text { Now I can be vulnerable and I can speak soft } \\
\text { and calm, I don't need to shout at everybody. }\end{array}$ & $\begin{array}{l}397- \\
399\end{array}$ \\
\hline Letting go & $\begin{array}{l}\text { I was like I don't want to leave but I know } \\
\text { that its the ending but that's actually taught } \\
\text { me endings can be healthy 'cause always } \\
\text { with relationships it's been like some big } \\
\text { drama and then I just never talked to them } \\
\text { again and then just go onto the next madness, } \\
\text { but here its ok for endings to happen, so } \\
\text { that's helped me }\end{array}$ & $\begin{array}{l}759- \\
768\end{array}$ \\
\hline Self reflection & $\begin{array}{l}\text { Ive learnt actually how to protect myself } \\
\text { more. So if im feeling that feeling, I } \\
\text { sit...identify and think, right, I can't be } \\
\text { feeling actually safe right now and try and } \\
\text { identify what it is and give my inner child the } \\
\text { acknowledgement, which is really important } \\
\text { to me as I still feel vulnerable }\end{array}$ & $\begin{array}{l}\text { 324- } \\
330\end{array}$ \\
\hline
\end{tabular}

SUBORDNIATE THEME: role of counsellor

\begin{tabular}{|l|l|l|}
\hline Subtheme 2 & Quote & Line \\
\hline Listened too & $\begin{array}{l}\text {..but for the first time she just understood and } \\
\text { I was like wow, I've never actually had } \\
\text { someone actually listen to me 'cause I had } \\
\text { difficulties with feelings. }\end{array}$ & $\mathbf{4 9 - 5 1}$ \\
\hline $\begin{array}{l}\text { Accessing feelings / } \\
\text { creative }\end{array}$ & $\begin{array}{l}\text { Really good, really safe and I was able to } \\
\text { express myself where I haven't before. It } \\
\text { took time. She was clever because she asked } \\
\text { me to think about things outside the therapy, }\end{array}$ & $\mathbf{8 9 - 9 1}$ \\
\hline
\end{tabular}


Helpful and unhelpful processes in psychological therapy for female substance users

\begin{tabular}{|c|c|c|}
\hline & $\begin{array}{l}\text { like art or songs and what they meant to me. } \\
\text { That really helped, especially getting out } \\
\text { things I couldn't say. She helped me feel the } \\
\text { feeling because im like, whats the feeling } \\
\text { behind the feeling. }\end{array}$ & \\
\hline & $\begin{array}{l}\text { The whole of my life was very } \\
\text { unmanageable. I didn't, I didn't ever used to } \\
\text { wash or anything and here it just taught me } \\
\text { how to... how to live, yeah, how to live again }\end{array}$ & $\begin{array}{l}\text { 203- } \\
207\end{array}$ \\
\hline Not judged & $\begin{array}{l}\text { I was sort of able to section it little bit by } \\
\text { little bit, and I know it didn't matter because } \\
\text { I was, oh, this is my truth, this is somebody } \\
\text { else's truth and now I now that...you now } \\
\text { how to find your own truth and it's ok to feel } \\
\text { the way I feel cause I used to feel shame for } \\
\text { feeling things and I was able to really open } \\
\text { up to her and be honest how I felt and not be } \\
\text { judged. }\end{array}$ & $\begin{array}{l}123- \\
134\end{array}$ \\
\hline $\begin{array}{l}\text { Staying in the here and } \\
\text { now }\end{array}$ & $\begin{array}{l}\text { She'd always bring me back into the room } \\
\text { when I'd be going off in different... all over } \\
\text { the place, she'd say " } \mathrm{S} \text { * bring yourself back' } \\
\text { and then always go back to my feelings } \\
\text { rather than all these crazy thoughts.... }\end{array}$ & $\begin{array}{l}188- \\
193\end{array}$ \\
\hline
\end{tabular}

SUBORDINATE THEME: IDENTIFICATION

\begin{tabular}{|l|l|l|}
\hline Subtheme 3: & Quote & Line \\
\hline Relating to other addicts & And then I used to find, I & $\mathbf{5 8 2}-$ \\
& think... when people talk about & $\mathbf{5 8 6}$ \\
& shame, I shut down and sort of & \\
& want to sleep. So I could & \\
& identify, oh well, I must have a & \\
& problem, I'm like them & \\
\hline
\end{tabular}


Helpful and unhelpful processes in psychological therapy for female substance users

\begin{tabular}{|c|c|c|}
\hline & $\begin{array}{l}\text { Just by being... being calm, } \\
\text { gentle, kind, compassionate } \\
\text { people, compassionate, and } \\
\text { identifying with me and me not } \\
\text { feeling I'm different of crazy. }\end{array}$ & $\begin{array}{l}877- \\
881\end{array}$ \\
\hline \multirow[t]{2}{*}{ Counsellor } & $\begin{array}{l}\text { Having a counsellor that was in } \\
\text { recovery also was good to know. } \\
\text { It made me realise we are all the } \\
\text { same, not everyone must be } \\
\text { crazy. }\end{array}$ & 543 \\
\hline & $\begin{array}{l}\text { Not everyones experience is the } \\
\text { same but she made me see there } \\
\text { is another side, there is hope }\end{array}$ & 341 \\
\hline MUSIC & $\begin{array}{l}\text { I thought music was going to } \\
\text { kick....I related music to } \\
\text { fighting cause when ive been in } \\
\text { the house its..but cause I wasn't } \\
\text { aware, I was just drunk and that } \\
\text { I'd sit there and people would } \\
\text { literally waste fire, so when I } \\
\text { heard it sober, I thought } \\
\text { maybe.....in the end Sam* got } \\
\text { me loving music and I'm } \\
\text { dancing, even at my graduation } \\
\text { day. I have music on all the time } \\
\text { now. }\end{array}$ & $\begin{array}{l}992- \\
1005\end{array}$ \\
\hline
\end{tabular}

PARTICIPANT 5: (JULES) psychodynamic

Subordinate theme: Emergence of new self and letting go of the old 
Helpful and unhelpful processes in psychological therapy for female substance users

\begin{tabular}{|c|c|c|}
\hline \multirow[t]{2}{*}{ Wanting to change } & $\begin{array}{l}\text {...there was a part of me that had to let go } \\
\text { to get well. Do you know? Put my life into } \\
\text { someone's hands and it happened to be my } \\
\text { counselor at the time. }\end{array}$ & $26-29$ \\
\hline & $\begin{array}{l}\text { It was my desire to get well as well. You } \\
\text { know what I mean? But was harder to open } \\
\text { up that I thought and actually do the work }\end{array}$ & $54-55$ \\
\hline \multirow[t]{2}{*}{$\begin{array}{l}\text { Resistance? Fighting } \\
\text { being cared for }\end{array}$} & $\begin{array}{l}\text { I can't remember but it was something to } \\
\text { do with erm, hmm, about caring, other } \\
\text { people, letting other people in and caring } \\
\text { for me. Erm, and I was like, 'Well, you're } \\
\text { just doing your job. You know.' She went, } \\
\text { 'Oh, really, J*? Oh no I would never care } \\
\text { for you, could i?' And that really touched } \\
\text { me, like you know, it just... }\end{array}$ & $\begin{array}{l}200- \\
207\end{array}$ \\
\hline & $\begin{array}{l}\text { My self esteem was on the floor how could } \\
\text { I, you know, I didn't like me so no-one, I } \\
\text { didn't believe that anybody could like me. } \\
\text { And then we linked it back, corr, don't get } \\
\text { me emotional. And we linked it back to } \\
\text { childhood that actually, that my mum and } \\
\text { dad hadn't really cared for me. }\end{array}$ & $\begin{array}{l}216- \\
221\end{array}$ \\
\hline Art / music & $\begin{array}{l}\text { It took a long time to be able to be open } \\
\text { about how I felt. Things like music and art I } \\
\text { hated at first. What was the point. Then I } \\
\text { realised that it was more about the meaning } \\
\text { behind that. So for music, that was a big } \\
\text { one for me, to chose a song that relates to a } \\
\text { subject and play it infront of others can be } \\
\text { scary, and can show a lot of you and say a } \\
\text { lot more than you ever could. Know what I } \\
\text { mean? I would listen to it in a session and } \\
\text { say that sums up how I feel right now. But }\end{array}$ & 289 \\
\hline
\end{tabular}


Helpful and unhelpful processes in psychological therapy for female substance users

\begin{tabular}{|l|l|l|}
\hline & $\begin{array}{l}\text { it felt my counsellor then knew what I } \\
\text { meant. Same with art, but im crap at } \\
\text { drawing (laughs) }\end{array}$ & \\
\hline
\end{tabular}

\section{Subordinate theme: Role of counsellor}

\begin{tabular}{|c|c|c|}
\hline Subtheme 2 & Quote & Line \\
\hline \multirow[t]{3}{*}{ Acceptance } & $\begin{array}{l}\text { I felt she understood, erm, and to some } \\
\text { degree she normalised it, like yeah, like } \\
\text { 'That's nothing j*. You know that's what } \\
\text { happens. Really?' You know, there was that, } \\
\text { I felt really erm, yeah safe. Yeah. }\end{array}$ & $64-67$ \\
\hline & $\begin{array}{l}\text { So she seemed to have, she..yeah..., she } \\
\text { could put what I was feeling into words } \\
\text { sometimes when I couldn't do that for myself } \\
\text { or I was too scared or too ashamed to }\end{array}$ & $78-80$ \\
\hline & $\begin{array}{l}\text { There were times when I thought my } \\
\text { behaviour was not normal and she, she, she } \\
\text { kind of erm, , yeah, reassured me that } \\
\text { 'Actually, do you know what } \mathrm{J}^{*} \text {, that is just } \\
\text { the process. }\end{array}$ & $85-88$ \\
\hline $\begin{array}{l}\text { Professional equally } \\
\text { safety }\end{array}$ & $\begin{array}{l}\text { Forget all the fluffiness about counselling. } \\
\text { You know what I mean? If you've got } \\
\text { anything to say to me, just please, can we just } \\
\text { deal with it? }\end{array}$ & $\begin{array}{l}253- \\
256\end{array}$ \\
\hline Identification? & $\begin{array}{l}\text { I think theres something about, 'Okay, I } \\
\text { know that this person is, erm, qualified. } \\
\text { They're skilled.' There is something about } \\
\text { that for me that makes me feel hopeful about } \\
\text { my own journey }\end{array}$ & $\begin{array}{l}274- \\
276\end{array}$ \\
\hline & $\begin{array}{l}\text { I explore things that I can't with just my } \\
\text { mates }\end{array}$ & \\
\hline
\end{tabular}


Helpful and unhelpful processes in psychological therapy for female substance users

\begin{tabular}{|c|c|c|}
\hline Counsellor in recovery & $\begin{array}{l}\text { I think that is massive.... Because I know } \\
\text { that, (pause), I don't know, I think erm, again } \\
\text { it was down to trust. It boils down to trust. }\end{array}$ & $\begin{array}{l}292- \\
298\end{array}$ \\
\hline & $\begin{array}{l}\text { Yeah, I mean, I know that there's good } \\
\text { counsellors, good therapists that are not in } \\
\text { recovery. I don't think it always matters. I } \\
\text { think once, when I know, I kind of feel more } \\
\text { safe because I know that they, they've been } \\
\text { through it and I can relate to them better then }\end{array}$ & 328 \\
\hline & She was someone I could learn from... & 746 \\
\hline
\end{tabular}

Participant 6 (Louise) CBT / Psychodynamic

Subordinate theme: Role of counsellor / cutting through the bullshit

\begin{tabular}{|c|c|c|}
\hline Subtheme 1 & Quote & Line \\
\hline Resistance & $\begin{array}{l}\text { The way...the way in..the way... well I told } \\
\text { you she could see through me.... } \\
\text { Yeah, because addicts are brilliant at } \\
\text { that... at being able to manipulate people } \\
\text { and if you can see through them... }\end{array}$ & $\begin{array}{l}670 \\
675\end{array}$ \\
\hline $\begin{array}{l}\text { Her attitude towards } \\
\text { counselling }\end{array}$ & $\begin{array}{l}\text { I needed someone to...to know when I was } \\
\text { lying, so I couldn't pull the wool over her } \\
\text { eyes. } \\
\text { Because I'd spent so much of my life sort of } \\
\text { lying to people and pulling the wool over } \\
\text { their eyes, as I say, that it was good to } \\
\text { realise that I wasn't on my own, and that } \\
\text { people could understand. }\end{array}$ & $\begin{array}{l}115- \\
122\end{array}$ \\
\hline $\begin{array}{l}\text { Impact of the counsellor - } \\
\text { helpful - creative / feelings }\end{array}$ & $\begin{array}{l}\text { Um...she..she..she had this great knack of } \\
\text { kind of noticing when I was trying to brush } \\
\text { over a subject, if I tried to use humour when } \\
\text { painful, which I did, you know. Also, we } \\
\text { worked on my feelings and what I was }\end{array}$ & $\begin{array}{l}440- \\
445\end{array}$ \\
\hline
\end{tabular}


Helpful and unhelpful processes in psychological therapy for female substance users

\begin{tabular}{|c|c|c|}
\hline & $\begin{array}{l}\text { avoiding. It was hard to open up. Some } \\
\text { weeks she would set me homework and I } \\
\text { had to draw how I felt then bring it in. I } \\
\text { wasn't feeling it at first, but looking back I } \\
\text { think it did help. But I couldn't get away } \\
\text { with anything (laughs) }\end{array}$ & \\
\hline \multirow[t]{2}{*}{$\begin{array}{l}\text { Feeling heard / } \\
\text { identification }\end{array}$} & $\begin{array}{l}\text { Because they will sit there, and they will } \\
\text { listen; they won't just talk non-stop about. } \\
\text { You know, it's not stuff that they learnt. It's } \\
\text { stuff that they...that they've experienced } \\
\text { themselves, most of them, and I find that } \\
\text { really helpful. Because you can really, sort } \\
\text { of like, when you know someone can } \\
\text { understand what you're saying it's a lot lot } \\
\text { lot more helpful. It also helps you to think } \\
\text { you have something in common. Your not } \\
\text { alone. }\end{array}$ & $81-87$ \\
\hline & $\begin{array}{l}\text { I find that my counsellor, she will just let } \\
\text { there be silences, and I know that's because } \\
\text { I know theres a reason behind that; theres } \\
\text { psychological things behind that. }\end{array}$ & $\begin{array}{l}264- \\
268\end{array}$ \\
\hline Direct - unhelpful & $\begin{array}{l}\text { Erm...she wasn't just...oh you know, } \\
\text { sympathising with me at all. It was 'cos that, } \\
\text { I think, I found I've had too much of that } \\
\text { wrapping me up in cotton wool, you know? } \\
\text { And she would just tell me straight. }\end{array}$ & $\begin{array}{l}193- \\
197\end{array}$ \\
\hline Unhelpful & $\begin{array}{l}\text { Counsellors in the past haven't wanted to } \\
\text { upset me, and things }\end{array}$ & $\begin{array}{l}203- \\
204\end{array}$ \\
\hline Unhelpful & $\begin{array}{l}\text { It felt like this is what I need; I need this. I } \\
\text { don't need someone to tell me what I want } \\
\text { to hear. }\end{array}$ & $\begin{array}{l}232- \\
234\end{array}$ \\
\hline Feeling understood & $\begin{array}{l}\text { Ive got a lot of things going on that } \\
\text { happened, and to know that she knew me }\end{array}$ & \\
\hline
\end{tabular}


Helpful and unhelpful processes in psychological therapy for female substance users

\begin{tabular}{|c|c|c|}
\hline & $\begin{array}{l}\text { well.... mean, 'cos in the past, counsellors } \\
\text { that ive met haven't known anything about } \\
\text { me; even the notes they haven't read. It } \\
\text { seemed different here. It wasn't just talking. }\end{array}$ & \\
\hline Needing consistency & $\begin{array}{l}\text { And I really really really wanna stress the } \\
\text { point about the counsellors leaving, because } \\
\text { I think that disrupted... }\end{array}$ & $\begin{array}{l}439- \\
441\end{array}$ \\
\hline Counsellor as an addict & $\begin{array}{l}\text { Because they had been there and done that, } \\
\text { you know...they'd experienced what you'd } \\
\text { experienced, be it with drink or drugs, } \\
\text { they'd been in those proper depths of } \\
\text { despair that you don't want to go back to. } \\
\text { You know what I mean? }\end{array}$ & $\begin{array}{l}551- \\
553\end{array}$ \\
\hline identification & $\begin{array}{l}\text { The woman was you know, together and } \\
\text { lovely, and everything I didn't know an } \\
\text { addict could have }\end{array}$ & 290 \\
\hline
\end{tabular}

\section{Participant 7 (Rachel): Gestalt}

\section{Subordinate theme: Emergence of new self}

\begin{tabular}{|c|c|c|}
\hline Subtheme 1: & Quotes & Lines \\
\hline Facing negative thoughts & $\begin{array}{l}\text { Cause I knew something was actually wrong } \\
\text { with me, there was so much stuff going on in } \\
\text { my past, that I'd never talked about, I'd } \\
\text { never exposed, it's never seen the light of } \\
\text { day. I just couldn't talk. And I think that was } \\
\text { the kind of main factor why I kept using, } \\
\text { because I was so frightened of actually who I } \\
\text { was, and what was actually inside of me, that } \\
\text { I had never, never expressed, I never told } \\
\text { anyone. }\end{array}$ & $23-28$ \\
\hline
\end{tabular}


Helpful and unhelpful processes in psychological therapy for female substance users

\begin{tabular}{|l|l|l|}
\hline & $\begin{array}{l}\text { I think that the voices in my head actually, } \\
\text { perhaps, weren't mine. You know, they was } \\
\text { very kind of condemning, very kind of } \\
\text { judgemental, very kind of hard thoughts, } \\
\text { which was a lot like my dad. So my internal } \\
\text { dialogue, and how I spoke to myself, how I } \\
\text { talked to myself. You know, and kind of, } \\
\text { bring that to the forefront. And she kind of } \\
\text { made me name that person, or that voice in } \\
\text { my head. I just believed I couldn't be helped } \\
\text { so what was the point? Its just another } \\
\text { attempt at recovery. Why do it? }\end{array}$ & 6 \\
\hline $\begin{array}{l}\text { You know, when I put down the drugs, I } \\
\text { kind if, you know, I get very needy, and very } \\
\text { lonely. You know, im kind of, I kind of tend } \\
\text { to use guys, you know, to fill the void in my } \\
\text { life. And I kind of really know where that } \\
\text { comes from now. }\end{array}$ & $46-49$ \\
\hline
\end{tabular}

Subordinate Theme: Moving forward

\begin{tabular}{|l|l|l|}
\hline Subtheme 2: & Quotes & Lines \\
\hline Releasing painful thoughts & $\begin{array}{l}\text { And we done some chair work, where erm, } \\
\text { you know, I had to actually tell my abuser } \\
\text { how I felt, and how angry I was. And I } \\
\text { found that difficult, you know, really } \\
\text { difficult. But I knew, if I kind of didn't get } \\
\text { this stuff out, it would be stuck in my brain } \\
\text { and then it would be stuck in my heart, you } \\
\text { know, and i. I'd be doomed to repeat the } \\
\text { same patterns. }\end{array}$ & 90 \\
\hline & $\begin{array}{l}\text { Any why just airing my feelings, and being } \\
\text { allowed, being allowed to express them, and }\end{array}$ & $204-$ \\
\hline
\end{tabular}


Helpful and unhelpful processes in psychological therapy for female substance users

\begin{tabular}{|c|c|c|}
\hline & $\begin{array}{l}\text { cry. It gave me a sense of, erm, being } \\
\text { okay.with them feelings. Being able to cry } \\
\text { and being able to show who I was, expose } \\
\text { myself for who I am. That was all part of the } \\
\text { process of accepting I'm an addict but im } \\
\text { going to be ok }\end{array}$ & \\
\hline anger & $\begin{array}{l}\text { You know its ok to be angry. Because anger } \\
\text { was all, for me, its okay to be angry, 'cause } \\
\text { anger was always kind of like, you know, I } \\
\text { could see people getting angry, and anger } \\
\text { would lead to violence. And I think that was } \\
\text { kind of why I was so, erm, afraid of getting } \\
\text { angry. But it just, it allowed me to realise } \\
\text { that anger is quite healthy }\end{array}$ & $\begin{array}{l}500- \\
550\end{array}$ \\
\hline \multirow[t]{2}{*}{ Resistance to acceptance } & $\begin{array}{l}\text { Because using drugs kind of dehumanised } \\
\text { me. I was very robotic, very angry, very, like } \\
\text { rigid, you know. I arrived and had all this } \\
\text { pent up emotion. And that was kind of like, } \\
\text { you know...I thawed out in here, that's the } \\
\text { word im looking for. I kind of thawed out } \\
\text { and began to see who *L really was, you } \\
\text { know flaws and assets you know. }\end{array}$ & $\begin{array}{l}223- \\
227\end{array}$ \\
\hline & $\begin{array}{l}\text { You know, my best, my best efforts got me } \\
\text { into a crack house in } b^{*} \text {, you know, that was } \\
\text { my safety, that's how safe I thought I was. } \\
\text { You know, im coming here, you know, and } \\
\text { just broke myself down, with the help of my } \\
\text { counsellor and the work we did, and try to } \\
\text { get a sense of who I was. }\end{array}$ & $\begin{array}{l}296- \\
301\end{array}$ \\
\hline Triggers & $\begin{array}{l}\text { You know, certain smells can trigger me, } \\
\text { you know, certain, just, just walking past a } \\
\text { house, loads of things trigger me, but im }\end{array}$ & $\begin{array}{l}400- \\
403\end{array}$ \\
\hline
\end{tabular}


Helpful and unhelpful processes in psychological therapy for female substance users

\begin{tabular}{|l|l|l|}
\hline & $\begin{array}{l}\text { kind of aware when they come up now, you } \\
\text { know. }\end{array}$ & \\
\hline Identification as addict & $\begin{array}{l}\text { And erm, it was such a relief when I, when I } \\
\text { could start identifying myself as an addict, } \\
\text { as well, all that kind of stuff. It was pure } \\
\text { relief. }\end{array}$ & 703 \\
\hline
\end{tabular}

Subordinate Theme: impact of counsellor

\begin{tabular}{|c|c|c|}
\hline Subtheme 3: & Quotes & Lines \\
\hline $\begin{array}{l}\text { Identification with addict } \\
\text { counsellor }\end{array}$ & $\begin{array}{l}\text { You know, it had happened to someone } \\
\text { else, you know, it was identification kind } \\
\text { of, oh yeah, yeah, I done that, im not alone. } \\
\text { And that was paramount for me, because } \\
\text { addiction is a lonely place. }\end{array}$ & 682 \\
\hline Shift in therapy & $\begin{array}{l}\text { I was using for such a long time, you know, } \\
\text { the places I went, and I was deprived, I } \\
\text { deprived myself. I starved myself of, of any } \\
\text { kind of light in my life. And when she said } \\
\text { that, that's paramount, that was one of the } \\
\text { breaking points. And I was like, I was } \\
\text { deprived, I deprived myself, cause I } \\
\text { abandoned myself. It took a while to see } \\
\text { that things could change. That meant I } \\
\text { could start seeing a new self. }\end{array}$ & $\begin{array}{l}626- \\
631\end{array}$ \\
\hline Using art / music & $\begin{array}{l}\text { You know, whats happened in this, in this } \\
\text { life. I tried to put it down on paper and } \\
\text { draw how I felt when she asked me to } \\
\text { express how I'd feel. You know with N*, } \\
\text { we could tie them up together, all these } \\
\text { emotions and kind of like, I could, I was } \\
\text { like, I could begin to feel whole. So many } \\
\text { little voices in my head, and so many little }\end{array}$ & $\begin{array}{l}759- \\
763\end{array}$ \\
\hline
\end{tabular}


Helpful and unhelpful processes in psychological therapy for female substance users

\begin{tabular}{|c|c|c|}
\hline & $\begin{array}{l}\text { characters dancing about, and I managed to } \\
\text { get them to one and music or art helped get } \\
\text { them to that place }\end{array}$ & \\
\hline \multirow[t]{2}{*}{ Professional } & $\begin{array}{l}\text { its done in such a structured, safe way, you } \\
\text { learn...I, I had to learn to actually trust } \\
\text { what was going on, and trust that these } \\
\text { professional counsellors know what they're } \\
\text { doing. And actually they do, because erm I, } \\
\text { ive stayed clean, im over a year clean. So } \\
\text { kind of like that, that speaks for itself. }\end{array}$ & $\begin{array}{l}261- \\
265\end{array}$ \\
\hline & $\begin{array}{l}\text { Because, you know it, it's like I, I wanted } \\
\text { her, to know her professional skills, cause } \\
\text { she did, it was such a, like..shes dealing } \\
\text { with me, isn't she, so I thought, oh well, I } \\
\text { must know who you are. But I liked that. . } \\
\text { You know, but she, you know, shes just } \\
\text { boundaries. And she said to me, *R, this } \\
\text { hour is for you and you only, it's nothing to } \\
\text { do with me, this is for you, you know, my } \\
\text { own hour. So I was like, oh my god, this is } \\
\text { for me, you know, a set time, yeah time for } \\
\text { me. }\end{array}$ & $\begin{array}{l}282- \\
288\end{array}$ \\
\hline \multirow[t]{2}{*}{ addict } & $\begin{array}{l}\text { I had an idea she was like me, an addict, } \\
\text { but wasn't sure. Then once session she said } \\
\text { something and I was like, she has been } \\
\text { here. She's been on this path. It made me } \\
\text { see her in a different light. I could relate } \\
\text { and the fact she survived gave me hope, } \\
\text { you know, made me think it will be ok }\end{array}$ & $\begin{array}{l}671- \\
672\end{array}$ \\
\hline & $\begin{array}{l}\text { But, you know, I believe that's a learned, a } \\
\text { learned brain pattern, what I had. You } \\
\text { know, what I hear, I heard in my childhood } \\
\text { stayed with me my adult life, you know. }\end{array}$ & $\begin{array}{l}136- \\
141\end{array}$ \\
\hline
\end{tabular}


Helpful and unhelpful processes in psychological therapy for female substance users

\begin{tabular}{|l|l|l|}
\hline And you know, she just talked me through \\
it, you know, in a gentle, I felt, gentle non \\
judgemental way, you know and just aired \\
what was going on in my head. Because it \\
was crazy in my mind, you know crazy \\
thoughts, you know. It was only once I \\
fnished therapy that I realised how much \\
she helped. Hope much she got me.
\end{tabular} \mid

Participant 8 (NOREEN): psychodynamic

\section{Subordinate theme: The process of therapy}

\begin{tabular}{|c|c|c|}
\hline SUBTHEME 1: counsellor & Quotes & Lines \\
\hline & $\begin{array}{l}\text { I was a bit wary of my first counsellor in } \\
\text { counselling because, my erm, relationship } \\
\text { with my counsellor at *R wasn't particularly } \\
\text { brilliant. I had a, a man there but obviously } \\
\text { it was my head space, he kind of just said to } \\
\text { me em alcohol isn't your problem, just read } \\
\text { like p.62 of this, its all about self- } \\
\text { centredness and self-will and I was like } \\
\text { what? So when I had counselling here, I } \\
\text { didn't see the point in opening up. I thought } \\
\text { it would all be the same again. }\end{array}$ & $64-69$ \\
\hline & $\begin{array}{l}\text { As counselling went on, i felt } \mathrm{C}^{*} \text { had time } \\
\text { for me and was prepared to listen and not } \\
\text { pass judegement and I always felt erm, that } \\
\text { I was just being totally read wrong really } \\
\text { from my original counsellor, that really } \\
\text { helped }\end{array}$ & $\begin{array}{l}197- \\
199\end{array}$ \\
\hline & $\begin{array}{l}\text { Basically having the trust, having the time } \\
\text { to erm, communicate without being }\end{array}$ & $\begin{array}{l}233- \\
235\end{array}$ \\
\hline
\end{tabular}


Helpful and unhelpful processes in psychological therapy for female substance users

\begin{tabular}{|c|c|c|}
\hline & $\begin{array}{l}\text { misheard and erm without being } \\
\text { misunderstood and we, and if the, we } \\
\text { always talked about I mean by things if I } \\
\text { brought something up, so there was no } \\
\text { chance of being misunderstood. But I only } \\
\text { realised later how much she had understood } \\
\text { all along }\end{array}$ & \\
\hline $\begin{array}{l}\text { Alternative forms of } \\
\text { expression - Art }\end{array}$ & $\begin{array}{l}\text { We had lots of moments, where I'd be just } \\
\text { sat there in silence and I'd always be trying } \\
\text { to work out what she was thinking, that was } \\
\text { my kind of, you know, way, and erm, she } \\
\text { asked me to use Art. I love art, that's erm } \\
\text { creative but I would, I took a while to show } \\
\text { her what I was doing and I feel quite } \\
\text { embarrassed about it now and erm, because } \\
\text { I had, I still have that perfectionism going } \\
\text { on for me as well. I kept destroying what } \\
\text { I'd made and showed her only selective bits } \\
\text { but I, look back now and I think if I'd only } \\
\text { just showed her what I had, as it meant she } \\
\text { could have really seen how I was feeling. }\end{array}$ & $\begin{array}{l}102- \\
110\end{array}$ \\
\hline & $\begin{array}{l}\text { Such as art, I could draw scenes or } \\
\text { scenarios. Ive always erm, spoke about erm } \\
\text { smells, colours, anything like that with } \\
\text { association or just how I felt } \\
\text { when....because i... when my feelings came } \\
\text { back, my head would not...I thought I was } \\
\text { going insane. }\end{array}$ & $\begin{array}{l}248- \\
252\end{array}$ \\
\hline
\end{tabular}

\section{Subordinate theme: Letting go and acceptance}


Helpful and unhelpful processes in psychological therapy for female substance users

\begin{tabular}{|c|c|c|}
\hline Leaving the past behind & $\begin{array}{l}\text { Yeah and I always erm, I just discussed } \\
\text { about my feelings which erm, was hard to } \\
\text { tell her about. I was honest with her but } \\
\text { took time, you know, and that was one of } \\
\text { the things that coming into recovery, I just } \\
\text { felt an instant weight lift where I didn't } \\
\text { have to lie anymore and that's one thing } \\
\text { that I made sure to the best of my ability I } \\
\text { just was honest but I did resist at first } \\
\text { (laughs) }\end{array}$ & $\begin{array}{l}152- \\
156\end{array}$ \\
\hline & $\begin{array}{l}\text { The kind of main factor I kept using was } \\
\text { because I was so frightened of actually who } \\
\text { I was, so I never expressed what was inside, } \\
\text { how would anyone understand? }\end{array}$ & 28 \\
\hline Counsellor understood & $\begin{array}{l}\text { I think I knew she was right (laughs). I } \\
\text { think I knew she was right and I didn't want } \\
\text { her to be. I just really didn't, you know. I } \\
\text { wanted to..to..you know, go of in this lala } \\
\text { land and everything would be ok..... }\end{array}$ & $\begin{array}{l}423- \\
431\end{array}$ \\
\hline retrospection & $\begin{array}{l}\text { I don't know, erm, just im amazed that ive } \\
\text { got that peace of mind, I really have that } \\
\text { calm and whatever, I don't know what left, } \\
\text { what left me, well I do know what left me } \\
\text { but I don't...I don't know exactly how it } \\
\text { left me. Just by being, I don't know whether } \\
\text { it was just by being honest and having } \\
\text { someone I could relate to. }\end{array}$ & $\begin{array}{l}281- \\
285\end{array}$ \\
\hline addict & $\begin{array}{l}\text { I had a feeling my counsellor was in } \\
\text { recovery and that did make a difference. I } \\
\text { was so big on trying to be honest, but it felt } \\
\text { easier when I knew. Little things gave it a } \\
\text { way. But she never said ether way. It just } \\
\text { meant I could be more open erm... I knew }\end{array}$ & 578 \\
\hline
\end{tabular}


Helpful and unhelpful processes in psychological therapy for female substance users

she wouldn't judge me cause she's wasn't that type of person 


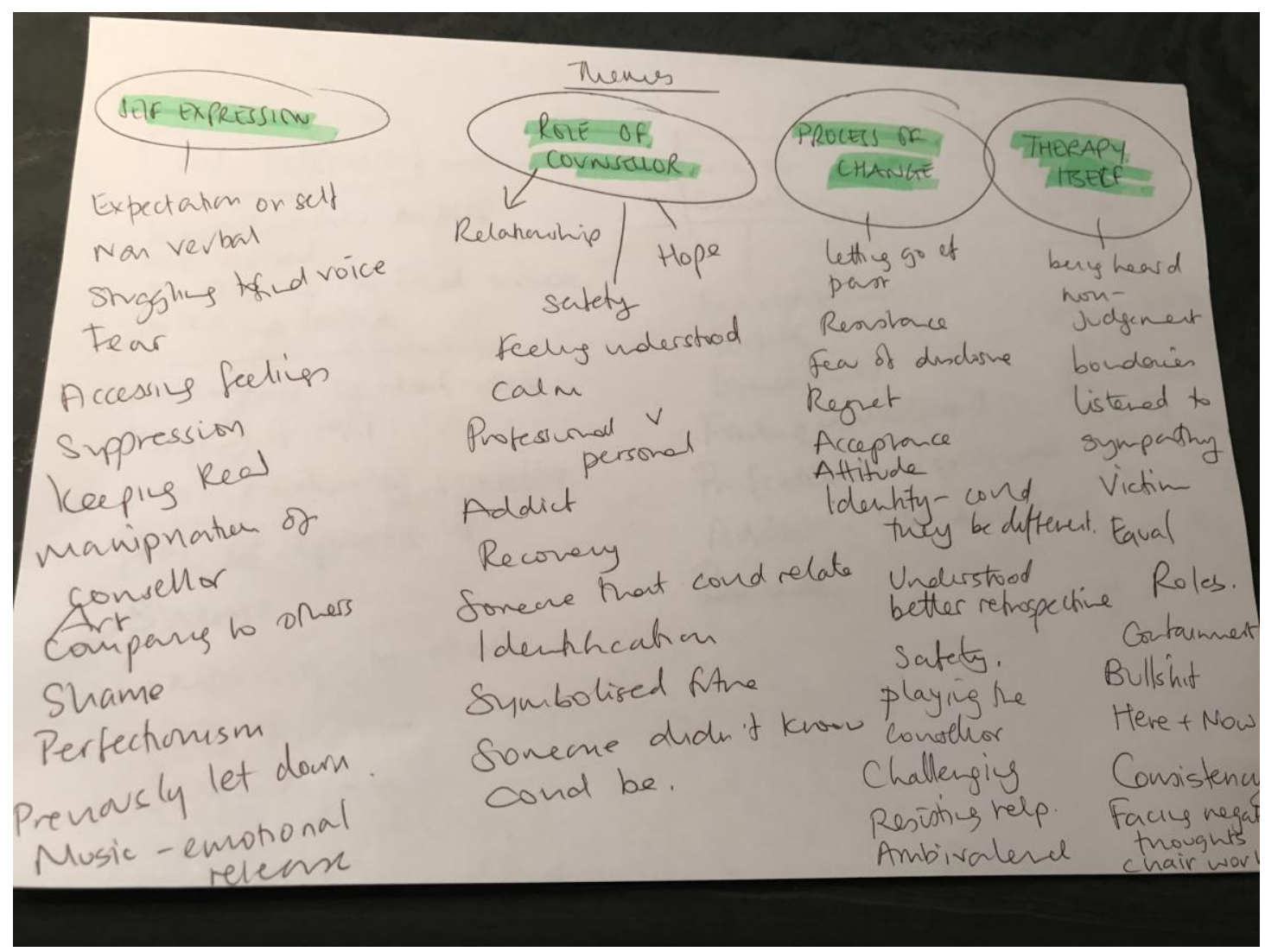

FINAL TABLE OF THEMES:

\section{(Appendix 10)}

\section{Superordinate Theme 1: What words can't express; finding another language}

\section{Subordinate themes: Overcoming the painful silence}

I really thought I was going to be able to come in and just talk and I couldn't. I absolutely could not (Sam, 134)

\section{Helping to identify feelings}

When you finish talking in a painful conversation, there's a painful silence; when you do it with art there's a product infront of you that collates all that, the good and bad times.... (Emma, 685) 
Helpful and unhelpful processes in psychological therapy for female substance users

\section{Superordinate Theme 2: Identification with my therapist \\ Subordinate themes: 'Been there, done that'}

Because they had been there and done that, you know....they'd experienced what you'd experienced, be it with drink or drugs, they'd been in those proper depths of despair that you don't want to go back to (Louise, 551-553)

\section{Symbolising hope and professionalism}

This woman was, you know, together and lovely, and everything I didn't know an addict could have (Louise, 290)

\section{Superordinate Theme 3: Getting towards acceptance}

\section{Subordinate themes: Resistance}

I wasn't completely honest with my counsellor. She went by the notes I'd selectively chosen to share. They were horrific enough. Um...but what I didn't tell her was that actually I wanted to give my body a break and use successfully (Jules, 103)

\section{'she had me all along'}

My triggers are guys, you know. I get lost very quickly, controlled and I'm unheard. My counsellor asked me to think about my relationship with men but I just wanted to ignore her. Only now I'm clean do I understand how right she was. (Emma, 789). 